\title{
WestVirginiaUniversity
}

THE RESEARCH REPOSITORY @ WVU

Graduate Theses, Dissertations, and Problem Reports

2013

\section{Multiwavelength Studies of Rotating Radio Transients}

Joshua J. Miller

West Virginia University

Follow this and additional works at: https://researchrepository.wvu.edu/etd

\section{Recommended Citation}

Miller, Joshua J., "Multiwavelength Studies of Rotating Radio Transients" (2013). Graduate Theses, Dissertations, and Problem Reports. 482.

https://researchrepository.wvu.edu/etd/482

This Dissertation is protected by copyright and/or related rights. It has been brought to you by the The Research Repository @ WVU with permission from the rights-holder(s). You are free to use this Dissertation in any way that is permitted by the copyright and related rights legislation that applies to your use. For other uses you must obtain permission from the rights-holder(s) directly, unless additional rights are indicated by a Creative Commons license in the record and/ or on the work itself. This Dissertation has been accepted for inclusion in WVU Graduate Theses, Dissertations, and Problem Reports collection by an authorized administrator of The Research Repository @ WVU.

For more information, please contact researchrepository@mail.wvu.edu. 


\title{
Multiwavelength Studies of Rotating Radio Transients
}

\author{
Joshua J. Miller
}

Dissertation submitted to the

Eberly College of Arts and Sciences

at West Virginia University

in partial fulfillment of the requirements

for the degree of

\author{
Doctor of Philosophy \\ in \\ Physics
}

Dr. Maura McLaughlin, Ph.D., Chair

Dr. Duncan Lorimer, Ph.D.

Dr. Loren Anderson, Ph.D.

Dr. Sean McWilliams, Ph.D.

Dr. Majid Jaridi, Ph.D.

Department of Physics and Astronomy

Morgantown, West Virginia

2013

Keywords: Pulsars; Neutron Stars

Copyright 2013 Joshua J. Miller 


\title{
ABSTRACT \\ Multiwavelength Studies of Rotating Radio Transients
}

\author{
Joshua J. Miller
}

Seven years ago, a new class of pulsars called the Rotating Radio Transients (RRATs) was discovered with the Parkes radio telescope in Australia (McLaughlin et al., 2006). These neutron stars are characterized by strong radio bursts at repeatable dispersion measures, but not detectable using standard periodicity-search algorithms. We now know of roughly 100 of these objects, discovered in new surveys and re-analysis of archival survey data. They generally have longer periods than those of the normal pulsar population, and several have high magnetic fields, similar to those other neutron star populations like the X-ray bright magnetars. However, some of the RRATs have spin-down properties very similar to those of normal pulsars, making it difficult to determine the cause of their unusual emission and possible evolutionary relationships between them and other classes of neutron stars.

We have calculated single-pulse flux densities for eight RRAT sources observed using the Parkes radio telescope. Like normal pulsars, the pulse amplitude distributions are well described by log-normal probability distribution functions, though two show evidence for an additional power-law tail. Spectral indices are calculated for the seven RRATs which were detected at multiple frequencies. These RRATs have a mean spectral index of $\left\langle\alpha_{\mathrm{SI}}\right\rangle=-3.2(7)$, or $\left\langle\alpha_{\mathrm{SI} \text { In }}\right\rangle=-3.1(1)$ when using mean flux densities derived from fitting log-normal probability distribution functions to the pulse amplitude distributions, suggesting that the RRATs have steeper spectra than normal pulsars. When only considering the three RRATs for which we have a wide range of observing frequencies, however, $\left\langle\alpha_{\mathrm{SI}}\right\rangle$ and $\left\langle\alpha_{\mathrm{SIln}}\right\rangle$ become $-1.7(1)$ and $-2.0(1)$, respectively, and are roughly consistent with those measured for normal pulsars. In all cases, these spectral indices exclude magnetar-like flat spectra. For PSR J1819-1458, the RRAT with the highest bursting rate, pulses were detected at 685 and $3029 \mathrm{MHz}$ in simultaneous observations and have a spectral index consistent with our other analysis.

We also present the results of simultaneous radio and X-ray observations of PSR J1819-1458. Our 94-ks XMM-Newton observation of the high magnetic field $\left(\sim 5 \times 10^{9} \mathrm{~T}\right)$ pulsar reveals a blackbody spectrum $(k T \sim 130 \mathrm{eV})$ with a broad absorption feature, possibly composed of two lines at $\sim 1.0$ and $\sim 1.3 \mathrm{keV}$. We performed a correlation analysis of the X-ray photons with radio pulses detected in 16.2 hours of simultaneous observations at $1-2 \mathrm{GHz}$ with the Green Bank, Effelsberg, and Parkes telescopes, respectively. Both the detected X-ray photons and radio pulses appear to be randomly distributed in time. We find tentative evidence for 
a correlation between the detected radio pulses and X-ray photons on timescales of less than 10 pulsar spin periods, with the probability of this occurring by chance being $0.46 \%$. This suggests that the physical process producing the radio pulses may also heat the polar cap. 


\section{Dedication}

The author wishes to dedicate this thesis to the memory of his brother

Jacob Michael Miller. 


\section{Acknowledgments}

"The way Josh is about to explain his projects will make them look straightforward and easy. In reality, these projects were nightmares... but they were nightmares in a good way."

-Dr. McLaughin's introduction for my oral defense.

The last few years have had many challenges and have only been possible with the support of the following people.

I would first like to thank Dr. Maura McLaughlin for her guidance during my research. She recruited me from her Computational Astrophysics class as someone with no prior astronomy experience and her expertise has led me to this point. Together we've delved into and teased out the technical details and challenges of research vistas never explored before. It seems like the unofficial motto of our weekly meetings had become, "We had no idea it would be this complicated!" (hence her quote above). Her patience has allowed me to go beyond the minimum effort required to publish a couple quick papers on RRATs, and do a thorough job of giving these new objects the diligence that they are owed.

I would like to thank my other committee members. Dr. Arthur Weldon laid down the foundation of my graduate physics career. Despite the rumors and horror stories I heard about his classes in hushed tones from other graduate students when I first arrived at WVU, his graduate quantum mechanics and electromagnetism courses were the most organized and enjoyable classes I have had the privilege of attending. Dr. Duncan Lorimer's well-organized classes gave a sense of continuity from Dr. Weldon's physics courses into the realm of radio astronomy. His graduate astronomy classes and perhaps a late night conversation at the 'Lucky Leprechaun' both helped develop my interest in radio astronomy. Next, I will never forget when Dr. Majid Jaridi almost did not sit on my committee for my oral exam. It was flattering to hear how impressive and intimidating my knowledge of astronomy was, but I am ultimately glad he decided to give me a chance and contributed as a member of my committee. I will never forget the conversations I had with Dr. Larry Halliburton in the spring of 2008; he had a great affect on the trajectory of my life and I can assuredly say that I would not be here if it were not for our discussions. Finally, my graduation would not be happening this semester without the help of Drs. Loren Anderson and Sean McWilliams, who were both willing to substitute on my defense committee at the last minute without hesitation.

I would also like to thank the many collaborators that have helped me tease out the nuances of my research journey. Specifically, my conversations with Drs. Evan Keane and Nanda Rea proved invaluable in my understanding of the nuances of both X-ray and radio observations and statistics; this dissertation is stronger as a result.

While the preceding people helped me form the 'big picture' presented in this dissertation, the following people were crucial with the day-to-day operation of my graduate education and research. Dr. Vlad Kondratiev introduced me to the world of linux. Post-docs Drs. Joris Verbiest, Paulo Freire, Manjari Bagchi, and Sam Bates provided sounding boards to help me work through computational, physics, 
astronomical, as well as LTEX problems. Nathan Garver-Daniels dropped what he is doing time after time to help me with my technical emergencies over the years, no matter how big or small the problem. Likewise, last-minute observation travel plans and (very importantly) payroll adjustments would not have been possible if it were not for the logistical help of Lori Hansen, Audrey Holsclaw, Alexandra Golubovic, and Megan Moore. Furthermore, I could not have achieved this accomplishment without the aid of Dr. Earl Scime, Vanessa Baker, Valerie Burgess, Siobhan Byrne, Devon Cleland, Sandy Johns, Amy Matuga, Greg Puskar, Sherry Puskar, and Phil Tucker. Particularly, I could not have maintained my status as a graduate student in the department after the passing of Jacob if it were not for the understanding of both Drs. McLaughlin and Scime.

Through my graduate education I have had the pleasure of sharing endless hours in cramped, windowless, fluorescent-lit offices with my colleagues. FIrst in an asbestos-filled old nuclear research retrofitted-restroom office in Hodges Hall with Eric, Herbert, Jeff, Jon, Mattias, Mike, Olga, Paul, Saikat, Sean, and Stephanie. Next it was the overcrowded, overheated, retrofitted-server room I shared with Ben, Colin, Jason, Josh R., Luke, Mitch, and Priya. Jason, Mitch, Pete and I were fortunate enough to share a couple windows in an office in the newly renovated White Hall for a few months, but it was not meant to be as Mitch, Pete, and I were ultimately assigned to share another windowless, albeit more spacious office together. I like to think all our friendships became more tight-knit under these conditions.

Whether it was a road trip, a game of trivia, hiking, a round of disc golf, a board game, a game of volleyball, helping me brew up a batch of beer, or just someone to sit on the barstool next to me, I also would like to thank my friends Aaron, Andrew, Audrey, Ben, Colin, Dave, Dusty, Eric, Fernando, James, Jason, Jeff, Jerry, Joe, John, Jon, Josh R., Katie, Kelly, Kristi, Luke, Mattias, Mitch, Mike, Nipuni, Paul, Pete, Ryan, Sam, Spencer, Stephanie, and Will. Thank you all for being there for me throughout this endeavor.

I would like to thank my parents, Robert and Sandra, who fostered an interest in math in me as well as the motivation to question things from an early age. Also, my siblings, Jacob and Cassy, who helped me become the person I am today.

Finally, a thank you to my partner-in-crime, Stephanie Sears; her love and support have made this graduate school undertaking sustainable. I am pleased we both chose the WVU Physics Department graduate program after EMU. Our adventures together have not stopped since. 


\section{Table of Contents}

List of Tables $\quad$ ix

List of Figures $\quad$ x

List of Abbreviations and Symbols $\quad$ xi

1 Introduction 1

1.1 Pulsar Fundamentals . . . . . . . . . . . . . . . . . . . . 2

1.1.1 Rotation Rates and Rotational Kinetic Energy . . . . . . . . . 2

1.1.2 Braking Index, Surface Magnetic Field, and Age . . . . . . . . 3

1.2 Interstellar Medium Effects . . . . . . . . . . . . . . . . . . . . 8

1.3 Pulsar Thermal Emission and Local Absorption Effects . . . . . . . . 11

1.4 The Pulsar Emission Mechanism . . . . . . . . . . . . . . . . . . 14

1.4.1 The Pulsar Magnetosphere . . . . . . . . . . . . . . . . . . . . 14

1.4.2 Radio Emission . . . . . . . . . . . . . . . . . . . . . . 18

1.4.3 High-Energy Emission . . . . . . . . . . . . . . . . 24

1.5 A Menagerie of Neutron Stars . . . . . . . . . . . . . . . . 29

1.5.1 Normal Pulsars . . . . . . . . . . . . . . . . . . 31

1.5.2 Millisecond Pulsars . . . . . . . . . . . . . . . 31

1.5.3 Giant Pulsing Pulsars . . . . . . . . . . . . . . . 32

1.5.4 Magnetars .................. . . 34

1.5.5 Nulling Pulsars and Rotating Radio Transients . . . . . . . . . 35

1.6 Outline of Dissertation . . . . . . . . . . . . . . . . . . . . . 38

2 RRAT Pulse Amplitude Distributions and Spectral Indices 39

2.1 Introduction . . . . . . . . . . . . . . . . . . . 39

2.2 Observations . . . . . . . . . . . . . . . . . . . 41

2.3 Analysis . . . . . . . . . . . . . . . . . . . 44

2.3.1 Flux Calculations ................. 44

2.3.2 Pulse Distributions . . . . . . . . . . . . . . . 50

2.3.3 Spectral Indices . . . . . . . . . . . . . . . . . . 53

2.3.4 Simultaneous Dual-Frequency Radio Observations of PSR J1819-1458 55

2.4 Discussion . . . . . . . . . . . . . . . . . . . . 57

3 Simultaneous X-ray and Radio Observations of Rotating Radio Transient J1819-1458 61

3.1 Introduction . . . . . . . . . . . . . . . . . . 61

3.2 X-Ray Observations and Analysis . . . . . . . . . . . . 65

3.2.1 Timing Analysis . . . . . . . . . . . . . . . . . 67

3.2.2 Spectral Analysis . . . . . . . . . . . . . . . . . 70

3.3 Radio Observations and Analysis . . . . . . . . . . . . . . . 75

3.4 Correlation of Radio Pulses and X-ray Photons . . . . . . . . . . . 77

3.5 Conclusions ..................... 84 


\section{List of Tables}

2.1 Spin-Down Properties for Eight RRATs. . . . . . . . . . . . . . . . . 42

2.2 Observing Parameters for Parkes Observations . . . . . . . . . . . . . 43

2.3 Flux Density Statistics . . . . . . . . . . . . . 46

3.1 X-ray Good Time Intervals . . . . . . . . . . . . . . . . . . 67

3.2 Spectral Fits for PSR J1819-1458 with EPIC-PN . . . . . . . . . . 74

3.3 Radio Parameters . . . . . . . . . . . . . . . . . . . 75 


\section{List of Figures}

1.1 Pulsar Emission Lighthouse Model . . . . . . . . . . . . . . . . . 7

1.2 Pulsar Magnetosphere . . . . . . . . . . . . . . . 16

1.3 Pair Cascade . . . . . . . . . . . . . . . . . . 21

1.4 Outer Gap Emission Model . . . . . . . . . . . . . . . . . 26

$1.5 P-\dot{P}$ Diagram. . . . . . . . . . . . . . . . . . 30

1.6 Series of Pulsar Single Pulses . . . . . . . . . . . . . . . . . . . . . . . . . . . . 32

1.7 The Spectrum of Pulsar Nulling. . . . . . . . . . . . . . . . 37

2.1 RRAT Pulse Profiles . . . . . . . . . . . . . . . . . . . . 48

2.2 RRAT Flux Density CCDFs . . . . . . . . . . . . . . 51

2.3 RRAT Spectral Indices . . . . . . . . . . . . . . 54

3.1 X-ray and Radio Profiles of PSR J1819-1458 . . . . . . . . . . . . . 69

3.2 X-ray Spectrum of PSR J1819-1458 . . . . . . . . . . . . . . . . . . 71

3.3 Cumulative X-ray Detections and Radio Pulses from PSR J1819-1458 78

3.4 PSR J1819-1458 X-Ray-Radio Detection Coincidences . . . . . . . 80

3.5 2XMMi J181928.8-145202 X-Ray-Radio Detection Coincidences . . 83 
List of Abbreviations and Symbols

\begin{tabular}{|c|c|}
\hline$\alpha$ & Angle Between Rotational and Magnetic Field Axes \\
\hline$\alpha_{\mathrm{SI}}$ & Spectral Index \\
\hline$\alpha_{\mathrm{SIGP}}$ & Spectral Index of Giant Pulses \\
\hline$\alpha_{\mathrm{SI} \ln }$ & Spectral Index from Log-Normal Distribution Fit \\
\hline$\beta$ & Impact Factor \\
\hline$\beta_{\text {dig }}$ & Digitization Sensitivity Loss Correction Factor \\
\hline$\gamma$ & Lorentz Factor \\
\hline$\gamma_{v}$ & Conversions by Collisions with Soft Photons \\
\hline$\gamma_{x}$ & Conversions by Collisions with X-rays \\
\hline$\epsilon_{0}$ & Permittivity of Free Space \\
\hline$\eta_{X}$ & X-ray Efficiency \\
\hline$\theta$ & Anglular Displacement from the Rotational Axis \\
\hline$\kappa_{\mathrm{SI}}$ & Log-Normal Distribution Scaling Factor for $\alpha_{\text {SI }}$ \\
\hline$\kappa_{\mathrm{SI} \ln }$ & Log-Normal Distribution Scaling Factor for $\alpha_{\text {SI ln }}$ \\
\hline$\mu_{\ln }$ & Mean of the Natural Logarithm of Flux Density \\
\hline$\nu$ & Rotational Frequency \\
\hline$\dot{\nu}$ & Rotational Frequency Derivative \\
\hline$\rho$ & Volume Charge Density \\
\hline$\sigma$ & Standard Deviation \\
\hline$\sigma_{1 \mathrm{~ms}}$ & Modified Radiometer Noise $\left(t_{\mathrm{samp}}=1 \mathrm{~ms}\right)$ \\
\hline$\sigma_{\ln }$ & Standard Deviation of the Natural Logarithm of Flux Density \\
\hline$\sigma_{\mathrm{H}}$ & Photoelectric Cross-Section \\
\hline$\sigma_{\mathrm{G}}$ & Gaussian Line Width \\
\hline$\sigma_{q}$ & Surface Charge Density \\
\hline$\sigma_{\mathrm{RN}}$ & Radiometer Noise \\
\hline$\sigma_{S}$ & Standard Deviation of Flux Density \\
\hline$\sigma_{\mathrm{SB}}$ & Stefan-Boltzmann Constant \\
\hline$\sigma_{S \ln }$ & Standard Deviation of Flux Density from Log-Normal Distribution Fit \\
\hline$\sigma_{w}$ & Standard Deviation of Pulse Widths \\
\hline$\tau$ & Neutron Star Age \\
\hline$\tau_{\mathrm{c}}$ & Characteristic Age \\
\hline$\tau_{\mathrm{G}}$ & Gaussian Line Depth \\
\hline$\tau_{\text {scat }}$ & Scattering Time \\
\hline$\chi^{2}$ & Reduced $\chi^{2}$ Statistic \\
\hline$\vec{\Omega}$ & Neutron Star Angular Velocity \\
\hline$\dot{\Omega}$ & Neutron Star Angular Speed Derivative \\
\hline$\omega$ & Photon Angular Frequency \\
\hline$\omega_{c}$ & Characteristic Photon Angular Frequency \\
\hline
\end{tabular}




\section{List of Abbreviations and Symbols (Cont....)}

\begin{tabular}{|c|c|}
\hline$A$ & Area \\
\hline$\vec{B}$ & Magnetic Field \\
\hline$B_{\text {crit }}$ & Critical Magnetic Field Strength \\
\hline$B_{\text {cy }}$ & Cyclotron Magnetic Field Strength \\
\hline$B_{\mathrm{S}}$ & Magnetic Field Strength at the Neutron Star Surface \\
\hline $\mathrm{BB}$ & Blackbody \\
\hline CCDF & Complementary Cumulative Distribution Function \\
\hline $\mathrm{CDF}$ & Cumulative Distribution Function \\
\hline$c$ & Speed of Light \\
\hline DISS & Diffractive Interstellar Scintillation \\
\hline DM & Dispersion Measure \\
\hline$d$ & Distance to the Neutron Star \\
\hline$d_{\text {cy }}$ & Cyclotron Absorption Depth \\
\hline$\vec{E}$ & Electric Field \\
\hline$E$ & Energy \\
\hline$E_{\text {cy }}$ & Cyclotron Absorption Energy \\
\hline$E_{\mathrm{G}}$ & Gaussian Line Energy \\
\hline$E_{p h}$ & Curvature Radiation Photon Energy \\
\hline$\dot{E}_{\text {dipole }}$ & Magnetic Dipole Radiation Power \\
\hline$\dot{E}_{\text {rot }}$ & Rotational Kinetic Energy Derivative \\
\hline$e^{2}$ & Elementary Charge \\
\hline$e^{+}$ & Positron \\
\hline$e^{-}$ & Electron \\
\hline $\mathrm{eV}$ & Electron Volt \\
\hline$F_{E}$ & Electric Force \\
\hline$F_{\text {emit }}$ & Emitted Spectrum Function \\
\hline$F_{\max }$ & Maximum Background-Corrected X-ray Pulse Profile Counts \\
\hline$F_{\min }$ & Minimum Background-Corrected X-ray Pulse Profile Counts \\
\hline$F_{\text {obs }}$ & Observed Spectrum Function \\
\hline$f$ & Frequency \\
\hline$\Delta f$ & Bandwidth \\
\hline$\Delta f_{\mathrm{DISS}}$ & Diffractive Interstellar Scintillation Frequency Scale \\
\hline$G$ & Gain \\
\hline GBT & Robert C. Byrd Green Bank Telescope \\
\hline GTI & Good Time Interval \\
\hline g & Gram \\
\hline H I & Neutral Hydrogen \\
\hline $\mathrm{Hz}$ & Hertz \\
\hline$h$ & Planck Constant \\
\hline$\hbar$ & Reduced Planck Constant \\
\hline$h_{\text {gap }}$ & Polar Gap Height \\
\hline hr & Hour \\
\hline
\end{tabular}




\section{List of Abbreviations and Symbols (Cont....)}

\begin{tabular}{|c|c|}
\hline$I$ & Moment of Inertia \\
\hline ISM & Interstellar Medium \\
\hline$\vec{J}$ & Current Density \\
\hline Jy & Jansky \\
\hline K & Kelvin \\
\hline$K$ & Flux Density Power-Law Exponent \\
\hline$k$ & Boltzmann Constant \\
\hline $\mathrm{KS}$ & Kolmogorov-Smirnov \\
\hline$L$ & Luminosity \\
\hline LGM-1 & Little Green Man 1 \\
\hline M & Mass \\
\hline$M_{\odot}$ & Solar Mass \\
\hline MJD & Modified Julian Day \\
\hline $\mathrm{m}$ & Meter \\
\hline$m$ & Particle Mass \\
\hline$m_{e}$ & Electron Mass \\
\hline$m_{p}$ & Protron Mass \\
\hline$\vec{m}$ & Magnetic Dipole Moment \\
\hline$N$ & Total Number of Charged Particles \\
\hline$N_{\text {chan }}$ & Number of Frequency Channels \\
\hline$N_{\mathrm{e}}$ & Number of Epochs \\
\hline$N_{\mathrm{p}}$ & Number of Pulses \\
\hline$N_{\text {pol }}$ & Number of Polarizations Summed \\
\hline NS & Neutron Star \\
\hline$n$ & Braking Index \\
\hline$n_{e}$ & Free Electron Number Density \\
\hline$n_{\mathrm{GJ}}$ & Goldreich-Julian Density \\
\hline$n_{\mathrm{H}}$ & Neutral Hydrogen Column Density \\
\hline$n_{\mathrm{Ne}}$ & Neon Column Density \\
\hline$n_{\mathrm{O}}$ & Oxygen Column Density \\
\hline$P$ & Rotational Period \\
\hline$\dot{P}$ & Rotational Period Derivative \\
\hline$P_{0}$ & Initial Rotational Period \\
\hline PDF & Probability Distribution Function \\
\hline PMPS & Parkes Multibeam Pulsar Survey \\
\hline PSR & Pulsar \\
\hline pc & Parsec \\
\hline$Q_{\mathrm{KS}}$ & Significance Level of KS Goodness-to-Fit Statistic \\
\hline$q$ & Electric Charge \\
\hline$R$ & Neutron Star Radius \\
\hline$\vec{R}_{c}$ & Magnetic Field Line Curvature Radius \\
\hline$R_{\mathrm{LC}}$ & Light Cylinder Radius \\
\hline
\end{tabular}




\section{List of Abbreviations and Symbols (Cont....)}

$\begin{array}{ll}\text { RFI } & \text { Radio Frequency Interference } \\ \text { RRAT } & \text { Rotating Radio Transient } \\ \vec{r} & \text { Position from Neutron Star Center } \\ \text { rad } & \text { Radian } \\ S & \text { Flux Density } \\ \langle S\rangle_{\text {ln }} & \text { Mean Flux Density from Log-Normal Distribution Fit } \\ \langle S\rangle_{\min } & \text { Minimum Mean Flux Density } \\ \mathrm{SG} & \text { Slot Gap } \\ \mathrm{S} / \mathrm{N} & \text { Signal-to-Noise Ratio } \\ \mathrm{S} & \text { Second } \\ s_{o} & \text { Field Coherence Length Scale } \\ \mathrm{T} & \text { Tesla } \\ T & \text { Temperature } \\ T_{\text {atm }} & \text { Atmosphere Temperature } \\ T_{\text {rec }} & \text { Receiver Temperature } \\ T_{\text {sky }} & \text { Sky Temperature } \\ T_{\text {sys }} & \text { System Temperature } \\ T_{\text {spill }} & \text { Spillover Noise Temperature } \\ \text { TPC } & \text { Two Pole Caustic } \\ t_{\text {eff }} & \text { Effective Sampling Time including Dispersive Time Resolution } \\ \Delta t_{\text {DISS }} & \text { Diffractive Interstellar Scintillation Timescale } \\ t_{\text {samp }} & \text { Sampling time } \\ V & \text { Electric Potential } \\ \vec{v}_{E} & \text { E-cross-B Drift Velocity } \\ \vec{v}_{R} & \text { Curvature Drift Velocity } \\ v_{\text {ISS }} & \text { Neutron Star Transverse Speed } \\ \text { W } & \text { Watt } \\ w & \text { Pulse Width } \\ w_{\text {cy }} & \text { Cyclotron Absorption Width } \\ y_{\mathrm{G}} & \text { Gravitational Redshift Factor } \\ \text { yr } & \text { Year } \\ z & \text { Distance from the Neutron Star Surface }\end{array}$




\section{Chapter 1}

\section{Introduction}

Pulsars were first discovered by University of Cambridge graduate student Jocelyn Bell in 1967. She worked with her thesis supervisor, Dr. Antony Hewish, to construct a radio telescope to observe radio-emitting quasars by using interplanetary scintillation. Interplanetary scintillation is the apparent fluctuation of radio emission intensity due to the diffraction of radio waves as they pass through the turbulent solar wind, similar to the interstellar scintillation discussed in Section 1.2. Compact radio sources such as quasars will scintillate more than extended radio sources, therefore this process can be exploited to select quasars out. Thus a telescope consisting of 2000 dipoles covering $\sim 2 \times 10^{4} \mathrm{~m}^{2}$ was constructed over two years and was then operated by Bell under the supervision of Hewish to observe the sky at $81.5 \mathrm{MHz}$ with a $1 \mathrm{MHz}$ bandwidth between $+50^{\circ}$ and $-10^{\circ}$ declination once every four days. Using four 3-track pen recorders, 96 feet of paper was recorded every night and hand-analyzed by Bell. On August 6, she discovered a pulsing signal on the paper, but Hewish believed it to be terrestrial radio-frequency interference (RFI). Nevertheless, subsequent recordings proved this signal was being detected four minutes earlier every night, in sync with sidereal time. Further investigation at higher time resolution and with a second telescope of this apparent point source showed that the recurring signal was not an instrumental effect and 
that it was originating from outside our solar system yet within our galaxy at $19 \mathrm{~h}$ $19 \mathrm{~m}$ right ascension, $+21^{\circ}$ declination, and repeated every 1.337 seconds (Hewish et al., 1968). This proved the source was not human-made, but left open the possibility of the source being made by an intelligent extraterrestrial civilization and the source was briefly dubbed "Little Green Man 1" (LGM-1). The source, now known as PSR B1919+21, like many other sources found soon afterward, was then quickly identified as natural phenomenon: a rotating neutron star, now known as a pulsar $(\mathrm{PSR})$.

\subsection{Pulsar Fundamentals}

Pulsars are rapidly-rotating highly magnetized neutron stars. Forming from the collapse of massive stars, Baade \& Zwicky (1934) proposed that these rapidly-

rotating (spin period, $P \sim 1 \mathrm{~s}$ ), highly magnetic $\left(B>10^{4} \mathrm{~T}\right)$, compact objects known as neutron stars could be formed in supernova explosions.

\subsubsection{Rotation Rates and Rotational Kinetic Energy}

Pulsars have rotational periods $(P)$ as short as $1.4 \mathrm{~ms}$ for PSR J1748-2446ad (Hessels et al., 2006) and as large as $11.8 \mathrm{~s}$ for PSR J1841-0456 (only seen at Xray wavelengths, see Vasisht \& Gotthelf, 1997; Kuiper et al., 2006). These rotation rates are not constant, however, and are observed to decrease over time. Pulsar's rotational period derivatives $(\dot{P})$ are typically non-zero and if intrinsic, are always positive. Sections 1.5.1 and 1.5.2 discuss the distributions of $P$ and $\dot{P}$ in further 
detail. The rate at which a pulsar's rotational kinetic energy changes with respect to the increase in its rotational period is

$$
\dot{E}_{\mathrm{rot}}=\frac{d}{d t} \frac{1}{2} I \Omega^{2}=I \Omega \dot{\Omega}=-4 \pi^{2} I \dot{P} P^{-3}
$$

where $\Omega=2 \pi / P$ is the angular rotational speed of the pulsar and $I$ is the pulsar's moment of inertia (assumed to be constant). This change in rotational kinetic energy is also known as the pulsar's spin-down luminosity and represents the energy loss of the star used to power the star's electromagnetic radiation (see Section 1.4).

If we treat the pulsar as a sphere of uniform density, then $I=\frac{2}{5} M R^{2}$, where $M$ and $R$ are the mass and radius of the pulsar, respectively. We can then use the canonical values of pulsar mass and radius, $M=1.4 M_{\odot}$ (Thorsett \& Chakrabarty, 1999 ) and $R=10 \mathrm{~km}$ (Lattimer \& Prakash, 2001), to get a canonical moment of inertia of $10^{38} \mathrm{~kg} \mathrm{~m}^{2}$. Plugging this value into Equation 1.1, we then have

$$
\dot{E}_{\mathrm{rot}}=4 \times 10^{24} \mathrm{~W}\left(\frac{\dot{P}}{10^{-15} \mathrm{~s} / \mathrm{s}}\right)\left(\frac{P}{\mathrm{~s}}\right)^{-3} .
$$

\subsubsection{Braking Index, Surface Magnetic Field, and Age}

The conservation of magnetic flux during the creation of a neutron star from the collapse of massive star results in a large surface magnetic field strength, $B_{\mathrm{S}}$, predicted to range from $10^{4}$ to $10^{10} \mathrm{~T}$. While we cannot directly measure the magnetic field of a pulsar, one way to infer its surface magnetic field strength from a pulsar's measured rotational period and measured change in rotational period is to 
assume its loss of rotational kinetic energy $\left(\dot{E}_{\text {rot }}\right)$ stems totally from magnetic dipole braking.

A magnetic dipole with moment $\vec{m}$ rotating at angular frequency $\Omega$ will radiate electromagnetic waves with radiation power

$$
\dot{E}_{\text {dipole }}=\frac{1}{6 \pi \epsilon_{0} c^{5}}|\vec{m}|^{2} \Omega^{4} \sin ^{2} \alpha
$$

where $\alpha$ is the angle between the rotational spin axis and the magnetic dipole axis, $\epsilon_{0}$ is the permittivity of free space, and $c$ is the speed of light. If we apply conservation of energy and assume all the energy radiated from the star comes from the loss of its rotational kinetic energy, i.e. $\dot{E}_{\text {dipole }}+\dot{E}_{\text {rot }}=0$, then Equations 1.1 and 1.3 therefore give us

$$
\dot{\Omega}=\left(\frac{|\vec{m}|^{2} \sin ^{2} \alpha}{6 \pi \epsilon_{0} I c^{5}}\right) \Omega^{3}
$$

which yields the power law relationship

$$
\dot{\nu} \propto \nu^{n}
$$

where $\nu=2 \pi \Omega=1 / P$ is the rotational frequency of the pulsar and $n$ is the braking index of the pulsar. Equation 1.4 implies a breaking index of $n=3$ for pulsars which are slowing down purely by energy loss due to magnetic dipole radiation. In practice, however, energy can be lost in other ways and measured braking indices vary from from $n=0.9$ to $n=2.9$ (Espinoza et al., 2011; Magalhaes et al., 2012). 
Equation 1.5 can also be rewritten in terms of the spin period as $\dot{P} \propto P^{2-n}$, which, as long as $n \neq-1$, can be solved to give the age of the pulsar,

$$
\tau=\frac{P}{(n-1) \dot{P}}\left(1-\left(\frac{P_{0}}{P}\right)^{n-1}\right)
$$

where $P_{0}$ was the spin period of the pulsar when it was born. If we assume the pulsar has slowed down considerably since its birth $\left(P \gg P_{0}\right)$ and $n=3$ as suggested by Equation 1.4, we have what is called the characteristic age of the pulsar

$$
\tau_{\mathrm{c}} \equiv \frac{P}{2 \dot{P}} \simeq 15.8 \operatorname{Myr}\left(\frac{P}{\mathrm{~s}}\right)\left(\frac{\dot{P}}{10^{-15} \mathrm{~s} / \mathrm{s}}\right)^{-1}
$$

If we relate the dipole magnetic field strength to its magnetic dipole moment as $B=|\vec{m}| / r^{3}$, we can find the surface magnetic field strength by solving Equation 1.4 for $|\vec{m}|$ and setting $r=R$, which yields

$$
B_{\mathrm{S}} \equiv B(r=R)=\sqrt{\frac{3 c^{5}}{32 \pi^{3} \epsilon_{0}} \frac{I}{R^{6} \sin ^{2} \alpha} P \dot{P}}
$$

Plugging in the canonical values of $R$ and $I$ discussed in Section 1.1.1, we can calculate the minimum canonical surface magnetic field strength by assuming the magnetic dipole axis is perpendicular to the pulsar's rotational axis $\left(\alpha=90^{\circ}\right)$, which leads to the relationship

$$
B_{\mathrm{S}}=3.2 \times 10^{15} \mathrm{~T} \sqrt{\frac{P}{\mathrm{~s}} \frac{\dot{P}}{\mathrm{~s} / \mathrm{s}}}
$$

Note that this relation is only practical as an order-of-magnitude estimate of the 
pulsar's magnetic field strength due to the number of assumptions going into its derivation, such as assuming the canonical values of $R$ and $M$ as well as assuming a uniform static dipolar magnetic field, the latter of which is almost certainly not the case in what is most likely a very dynamic and complicated system.

If the star's dipole magnetic field axis is misaligned from is rotation axis $(\alpha \neq$ $0^{\circ}$ ) and crosses the Earth's line-of-sight, electromagnetic emission from the rotating body can typically seen be seen as a series of pulses. This is illustrated by the lighthouse model shown in Figure 1.1.

The electric field induced by the rotating magnetic field fills the pulsar's magnetosphere with a plasma that corotates with the neutron star (further details are discussed in Section 1.4.1). As shown in Figure 1.1, there is a point where the plasma's tangential velocity is equal to the speed of light; this cylinder is defined as the light cylinder and has radius $R_{\mathrm{LC}}=c / \Omega=c P / 2 \pi$. The plasma and induced fields within the light cylinder co-rotate with the pulsar allowing the dipolar magnetic fields to create closed loops. Any dipolar magnetic fields that extend past the light cylinder radius cannot create closed loops. We therefore categorize the former and latter magnetic field lines into open field lines and closed field lines, respectively. Tracing the open field lines back to their footprints on the neutron star surface defines a pulsar's polar cap region. 


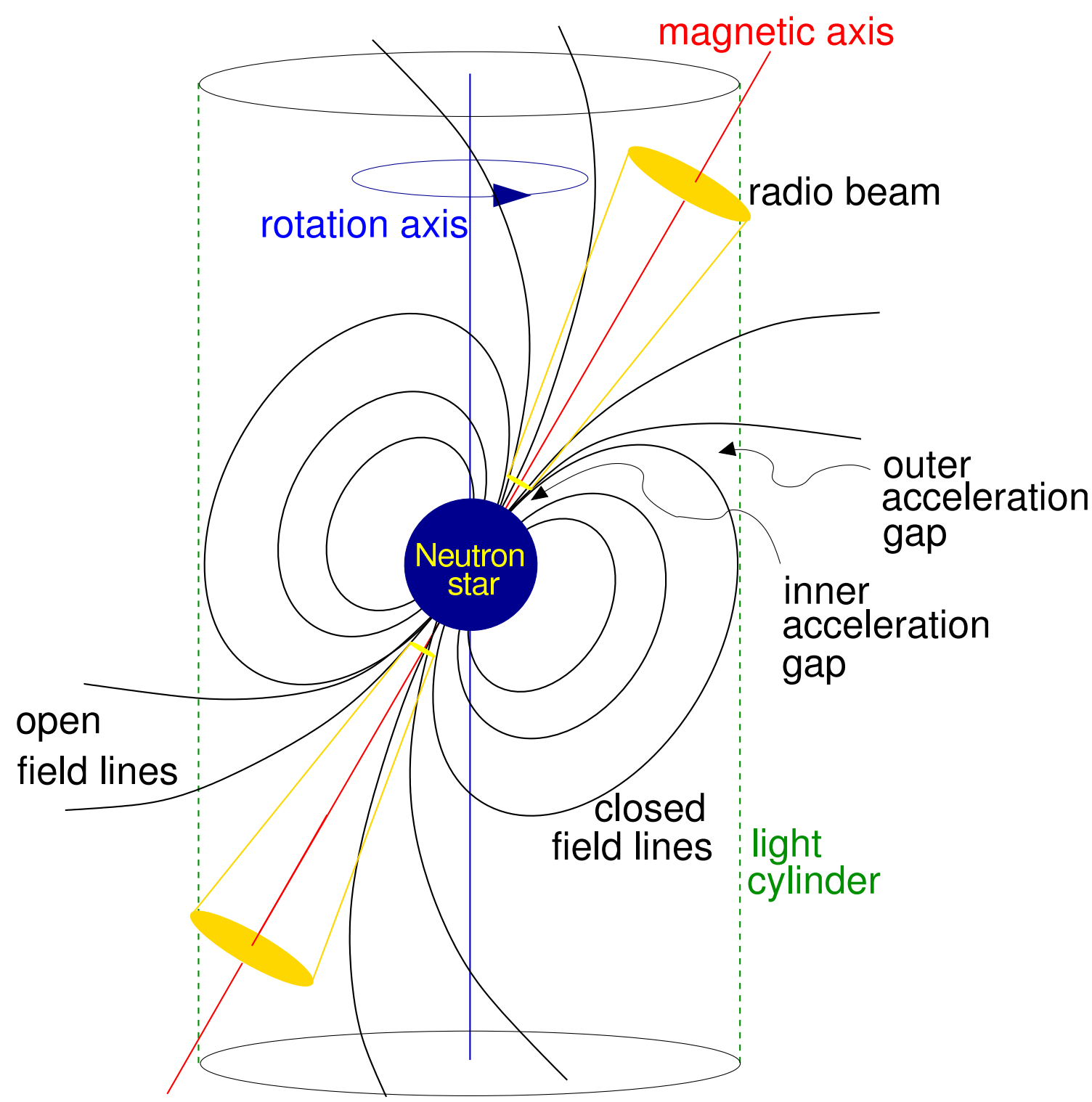

Figure 1.1: When not aligned with the rotation axis, emission from the open field lines of the pulsar's dipole magnetic field projects along the line-of-sight toward an observer as a series of periodic pulses. The light cylinder is defined by the radius from the neutron star at which the co-rotating tangential velocity is equal to the speed of light. Therefore, any magnetic field lines extending past the light cylinder cannot be closed field lines. The inner and outer acceleration gaps are gaps in the magnetosphere plasma that allows the presence of a non-zero electric field which accelerates charged particles and creates electromagnetic radiation, as shown in Section 1.4.1. (Lorimer \& Kramer, 2005) 


\subsection{Interstellar Medium Effects}

The interstellar medium (ISM) is mostly composed of a partially-ionized lowdensity cold-temperature hydrogen plasma. Electromagnetic waves radiated from a pulsar traveling through the ISM will have a frequency-dependent delay which is dependent on the total number of free electrons of the ISM, which will depend on the distance between the pulsar and Earth. This quantity is known as the Dispersion Measure (DM), defined by the integral

$$
\mathrm{DM}=\int_{0}^{d} n_{e} \mathrm{~d} l
$$

where $d$ is the distance to the pulsar, $n_{e}$ is the free electron density along the line of sight to the pulsar, and DM is typically expressed in units of $\mathrm{pc} \mathrm{cm}^{-3}$. If the arrival time of a single pulse is measured at two different frequencies, the time delay between the two frequencies due to the dispersion, $\Delta t$, will be related to the frequency-dependent nature of the ISM by

$$
\Delta t=\left(4.15 \times 10^{6} \mathrm{~ms}\right) \times\left[\left(\frac{f_{1}}{\mathrm{MHz}}\right)^{-2}-\left(\frac{f_{2}}{\mathrm{MHz}}\right)^{-2}\right] \times\left(\frac{\mathrm{DM}}{\mathrm{pc} \mathrm{cm}^{-3}}\right)
$$

where $f_{1}$ and $f_{2}$ are the lower and higher frequencies, respectively. Since $f_{1}$ and $f_{2}$ are known and $\Delta t$ can be measured, this equation can be used to find the DM of a pulsar. Once the DM of the pulsar is known, telescope data collected can be dedispersed by adding a time-delay to each frequency channel using Equation 1.11. 
In addition, a model for the free electron density in our Galaxy (e.g. Cordes \& Lazio, 2002) can be used to estimate $n_{e}$ along the line of sight to the pulsar, and then a determination of the pulsar's distance may be estimated using Equation 1.10.

Besides dispersion, the ISM can also scatter the radio waves passing through it. This gives the waves different path lengths between the pulsar and Earth, broadening the pulse shape which degrades our resolution of each pulse's arrival time over a scattering time, $\tau_{\text {scat }}$. While this dissertation does not set out to study the effects of ISM scattering, it must be taken into consideration to better understand the reliability of our pulse arrival times. An empirical relationship for $\tau_{\text {scat }}$ is (Bhat et al., 2004)

$$
\begin{array}{r}
\log _{10}\left(\frac{\tau_{\mathrm{scat}}}{\mathrm{ms}}\right)=-6.46+0.154 \log _{10}\left(\frac{\mathrm{DM}}{\mathrm{pc} \mathrm{cm}^{-3}}\right)+1.07\left(\log _{10}\left(\frac{\mathrm{DM}}{\mathrm{pc} \mathrm{cm}^{-3}}\right)\right)^{2} \\
-3.86 \log _{10}\left(\frac{f}{\mathrm{GHz}}\right)
\end{array}
$$

It can be seen that there is greater scattering associated with pulsars with a higher DM since it is related to both the distance and the free electrons the waves encounter in the ISM. In addition, Equation 1.12 shows that the scattering time decreases as the observation frequency increases.

Like scattering, diffractive scintillation by the ISM should also be taken into consideration to understand the quality of our time-varying pulse amplitude measurements. Scintillation is not too dissimilar from the twinkling of optical stars; it is the time-varying and frequency-dependent amplitude variation from the constructive 
and destructive electromagnetic wave interference stemming from the turbulence and varying density of the ISM. Scintillation can be categorized into two types: diffractive and refractive. Of the two types, diffractive interstellar scintillation (DISS) has the greatest potential to affect the quality of the time- and frequency-dependent amplitude measurements presented in this dissertation because refractive interstellar scintillation acts on a much larger timescale. DISS affects the amplitude of the pulses on the timescale

$$
\Delta t_{\mathrm{DISS}}=\frac{s_{o}}{v_{\mathrm{ISS}}}
$$

where $s_{o}$ is the field coherence length scale and $v_{\text {ISS }}$ is the transverse speed of the pulsar. Likewise, DISS has a frequency scale of

$$
\Delta f_{\mathrm{DISS}}=\frac{1.16}{2 \pi \tau_{\mathrm{scat}}}
$$

for a Kolmogorov spectrum. Therefore, the reliability of our ability to measure the scintillation bandwidth and timescale of the pulsar requires that the time resolution of the observations be finer then $\Delta t_{\text {DISS }}$.

In addition to the effects of the ionized component of the ISM, the non-ionized component, which typically has a much higher density, can also affect the passing electromagnetic waves. After the X-ray spectrum of the pulsar is emitted, $F_{\text {emit }}(E)$, it will then be subjected to photoelectric absorption as it travels through the ISM. This absorption is related to the neutral hydrogen column density, $n_{\mathrm{H}}$, along the line-of-sight so that the observed spectrum, $F_{\text {obs }}(E)$, is 


$$
F_{\text {obs }}(E)=F_{\text {emit }}(E) e^{-n_{\mathrm{H}} \sigma_{\mathrm{H}}(E)}
$$

where $\sigma_{\mathrm{H}}(E)$ is the energy dependent photoelectric cross-section, not including Thomson scattering. The cross-sections of Bałucińska-Church \& McCammon (1992) are used in this dissertation. Furthermore, abundances of other elements such as Oxygen and Neon can have a similar energy-dependent affect on the X-ray spectrum and are also taken into consideration in Chapter 3 using the solar abundances of Lodders (2003).

\subsection{Pulsar Thermal Emission and Local Absorption Effects}

Once the pulsar's distance and its detected pulse amplitude, i.e. flux density $S$, are both known, then an estimate of the pulsar's luminosity $L$ may be made. In general, a star's flux density is related to its luminosity as $S=L / A$, where $A$ is the area the luminosity is emitted over;

$$
A=4 \pi d^{2}
$$

for a sphere with radius $d$, where $d$ is distance between the pulsar and the observer, as before.

The pulsar also acts as a blackbody emitter with its luminosity given by the Stefan-Boltzmann law, which for a sphere with radius $R$ is given by 


$$
L=4 \pi R^{2} \sigma_{\mathrm{SB}} T^{4},
$$

where $T$ is the temperature of the pulsar, and $\sigma_{\mathrm{SB}}$ is the Stefan-Boltzmann constant. If a blackbody emission model can be fitted to the emission spectrum of a pulsar, typically in the X-ray spectrum, the fitted temperature can then be used to estimate the radius of the pulsar using Equations 1.16 and 1.17,

$$
R=\frac{S d^{2}}{\sigma_{\mathrm{SB}} T^{4}} .
$$

Equation 1.18 can also be used to estimate the radius of a "hot-spot" of thermal emission, at a second higher temperature, at the polar cap of the pulsar for small opening angles. In the case of two overlapping blackbody spectra, there will be an apparent dip in the combined spectrum where the hotter blackbody spectra begins to dominate at higher energies. This effect can look similar to an absorption within a single blackbody spectrum.

Aside from combining blackbodies, another reason for the apparent dip in the spectrum could be absorption. As already mentioned in Section 1.2, elements within the ISM can absorb and scatter waves at certain energies. But before the ISM can even affect a pulsar's emitted waves, electromagnetic emission may also be emitted/absorbed by the magnetized plasma surrounding the neutron star itself. Absorption observed in the X-ray spectrum of a pulsar may be due to resonant cyclotron scattering, which is the mechanism where charged particles spiral around magnetic field lines absorbing and emitting electromagnetic radiation. Cyclotron 
radiation has a spectrum spiking at the fundamental frequency (or energy) of the particles' gyro-orbit, with harmonics at higher integer-multiples of the fundamental frequency. The absorptions at harmonic frequencies are typically of lower intensity and can be more difficult to detect. Imperfections in the actual emission and absorption environment, e.g., non-uniformities in the magnetic field and collisions, can cause broadening of the spectral lines. When only considering the fundamental cyclotron frequency, the absorption can be modeled as

$$
F_{\text {obs }}(E)=F_{\text {emit }}(E) e^{-d_{\mathrm{cy}} \frac{\left(w_{\mathrm{cy}} E / E_{\mathrm{cy}}\right)^{2}}{\left(E-E_{\mathrm{cy}}\right)^{2}+w_{\mathrm{cy}}^{2}}}
$$

where $d_{\text {cy }}$ is the absorption depth, $w_{\text {cy }}$ is the width of the absorption due to broadening, and $E_{\mathrm{cy}}=h q B / 2 \pi m$ is the cyclotron energy; where $q$ is the electric charge, $m$ is the charged particle's mass, and $h$ is the Planck constant, see e.g. Mihara et al. (1990). Finally, if the cause of absorption is unknown, an empirical Gaussian function may also be fit to the absorption,

$$
F_{\text {obs }}(E)=F_{\text {emit }}(E) e^{-\frac{\tau_{\mathrm{G}}}{\sqrt{2 \pi} \sigma_{\mathrm{G}}} e^{-\frac{\left(E-E_{\mathrm{G}}\right)^{2}}{2 \sigma_{\mathrm{G}}^{2}}}},
$$

where $E_{\mathrm{G}}$ is the line energy, $\sigma_{\mathrm{G}}$ is the line width, and $\tau_{\mathrm{G}}$ is the line depth so that the optical depth at the center line of the absorption is $\tau_{\mathrm{G}} /\left(\sqrt{2 \pi} \sigma_{\mathrm{G}}\right)$. 


\subsection{The Pulsar Emission Mechanism}

The pulsar environment is very complicated and not well understood. That being said, simple models of the pulsar and its magnetosphere can explain some basic phenomena and give some insight to the structure of pulsar emission. One of the goals of this dissertation is to help test the predictions of some of the pulsar emission models which provide falsifiable results.

\subsubsection{The Pulsar Magnetosphere}

Following the pulsar electrodynamic analysis done by Goldreich \& Julian (1969), we will begin by modeling a neutron star as a conducting sphere rapidly rotating within a vacuum (Deutsch, 1955). If we consider the dipolar magnetic field of the pulsar mentioned in Section 1.1.2, in the case where it is not aligned with the rotation axis $\left(\alpha \neq 0^{\circ}\right)$, which is required for observable pulses, then the pulsar will be polarized and an induced electric field will be created at every position $\vec{r}$, i.e., $(\vec{\Omega} \times \vec{r}) \times \vec{B}$. The charge density within the conductor will therefore redistribute itself to create an electric field, $\vec{E}$, so that the Lorentz force is zero, which is satisfied by the condition

$$
\vec{E}+(\vec{\Omega} \times \vec{r}) \times \vec{B}=0
$$

Laplace's equation can then be solved for the electrostatic potential, $V$, within the vacuum surrounding the conducting sphere with the permittivity of free space, $\epsilon_{0}$. The electrostatic potential must then satisfy the boundary condition of being 
continuous at the neutron star surface, $r=R$. The solution is a quadrupole field,

$$
V(r, \theta)=-\frac{B_{\mathrm{S}} \Omega R^{5}}{6 r^{3}}\left(3 \cos ^{2} \theta-1\right)
$$

where $(r, \theta)$ are polar coordinates centered on the neutron star's center with $\theta$ measured from the rotation axis $\vec{\Omega}$. The discontinuity of the component of the electric field normal to the neutron star surface then governs what the surface charge density must be,

$$
\sigma_{q}(r=R, \theta)=-\epsilon_{0} B_{\mathrm{S}} \Omega R \cos ^{2} \theta
$$

The component of the electric field parallel to the magnetic field at the neutron star surface is therefore,

$$
\left.E_{\|}(r=R) \equiv \frac{\vec{E} \cdot \vec{B}}{B}\right|_{r=R}=-\Omega B_{\mathrm{S}} R \cos ^{3} \theta
$$

The resulting electric force acting on charged particles on the surface of the neutron star, $F_{E}=q E_{\|}$, is much greater than the opposing gravitational force. As a result, the vacuum surrounding the neutron star will quickly be replaced by a highly-magnetized plasma; this region is defined as the pulsar's magnetosphere. For the steady-state case, we can treat the plasma as another conductor which will redistribute the charge particles as

$$
\rho(r, \theta)=\epsilon_{0} \vec{\nabla} \cdot \vec{E}=-2 \epsilon_{0} \vec{\Omega} \cdot \vec{B}=-\frac{\epsilon_{0} B_{0} \Omega R^{3}}{r^{3}}\left(3 \cos ^{3} \theta-1\right),
$$




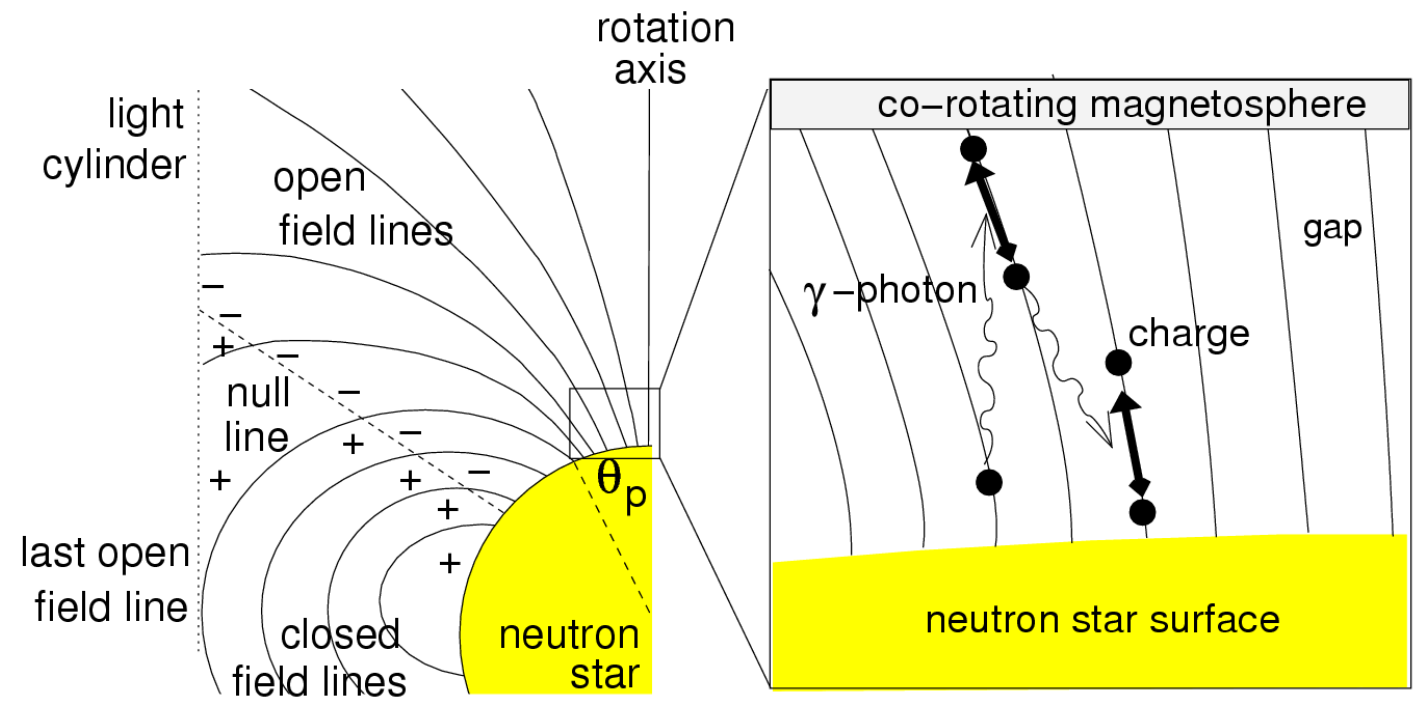

Figure 1.2: The Goldreich-Julian pulsar magnetosphere model. The existence of the polar gap developed by this model is indicated. (Lorimer \& Kramer, 2005)

so that they screen the electric field within the magnetosphere to create another relatively force-free condition, $\vec{E} \cdot \vec{B} \approx 0$. The surface swept by the angle where Equation 1.25 changes $\operatorname{sign}(\cos \theta=1 / \sqrt{3})$ is denoted as the "null-charge surface", where it leads from $\vec{E}=0$ that $\vec{\Omega} \cdot \vec{B}=0$ (see Figure 1.2). Assuming the plasma is fully ionized ( $n_{e}=\rho / e$, where $e$ is the electric charge of an electron), the number density at the magnetic pole is then its maximum possible value given this analysis and is known as the "Goldreich-Julian (GJ) density",

$$
n_{\mathrm{GJ}}=\frac{-2 \epsilon_{0} \Omega B}{e} \approx 7 \times 10^{10} \mathrm{~cm}^{-3}\left(\frac{P}{\mathrm{~s}}\right)^{-1 / 2}\left(\frac{\dot{P}}{10^{-15} \mathrm{~s} / \mathrm{s}}\right)^{1 / 2}
$$

The other component of the electric field which is perpendicular to the magnetic field causes what is referred to as "E-cross-B drift" in the plasma, $\vec{v}_{E}=$ $(\vec{E} \times \vec{B}) / B^{2}$, and causes the magnetosphere to corotate with the pulsar. Unlike 
guiding center drifts caused by non-electric forces perpendicular to the magnetic field, the electric field does not create a net current within the plasma since the perpendicular electric force on each particle also depends on its charge and mass, resulting in electrons and ions having the same velocity. As mentioned in Section 1.1.2, while it is possible for the closed magnetic field lines to corotate with the pulsar within the light cylinder, charged particles can escape along the open field lines above the magnetic polar caps, which extend outside of the pulsar's light cylinder. This region happens to be the region of highest density predicted by the above analysis, the GJ density. As the plasma density over the polar cap is depleted by this process, quasi-neutrality within the plasma is no longer maintained which once again creates an unbalanced electric field, $\vec{E} \cdot \vec{B} \neq 0$. This electric field accelerates charged particles along the magnetic field lines, producing electromagnetic radiation.

Emission mechanisms require $n_{e} \gg n_{\mathrm{GJ}}$. This motivates the need for regions of higher density than given in our model thus far, this is explored in Sections 1.4.2 and 1.4.3. The regions in which our above analysis breaks down, i.e. the plasma is no longer corotating with the pulsar and an unbalanced electric field brings about electromagnetic radiation, are thought to be the main regions of electromagnetic emission and are known as acceleration gaps. The two chief acceleration gaps are known as the inner and outer magnetosphere gap. The inner magnetosphere gap is located above the polar cap, and therefore also referred to as the polar gap. The outer magnetosphere gap is located between by the light cylinder near the cut-off between the open and closed field lines shown in Figure 1.2. 


\subsubsection{Radio Emission}

The model of a cone-shaped emission beam centered on the dipolar magnetic axis motivated in the previous section predicts a narrow pulse profile. In addition, observations of radio pulses have brightness temperatures of about $10^{25}-10^{30} \mathrm{~K}$; the only way to account for this is if the emission mechanism is coherent. Three emission models that can account for coherent emission are the antenna mechanism, relativistic plasma emission, and maser emission. In each case, plasma travels up from the neutron star surface along the magnetic field lines into the acceleration gaps where the charged particles then emit radiation tangent to the magnetic field lines. Here I will summarize the analysis of the antenna mechanism within the polar gap done by Ruderman \& Sutherland (1975), and then I will briefly relate the latter two coherent emission mechanisms to the discussion.

In an emission region, if there are $N$ charged particles, each with charge $q$ which are emitting incoherently, then it follows that the emitted power will be $N$ times the power of a single emitting charged particle. In an antenna mechanism model, the charged particles are confined within a volume of a length-scale which is smaller than half of the wavelength of each particle's emission. In this case, all the particles will act in phase as one single emitting particle with charge $N q$ and the emitted power will instead be $N^{2}$ times the power of a single emitting charged particle.

As mentioned in Section 1.4.1, such an emission region could form over the polar cap, i.e. the polar gap, due to charged particles escaping along the open 
field lines and hence depleting the plasma density to create an environment where $\vec{E} \cdot \vec{B} \neq 0$. Within the polar gap, the component of the electric field parallel to the magnetic field as a function of distance from the neutron star surface, $z$, is

$$
E_{\|}=2 \Omega B_{\mathrm{S}}\left(h_{\text {gap }}-z\right)
$$

where $h_{\text {gap }}$ is the total polar gap height. The electric potential across the entire gap is therefore

$$
\Delta V=\Omega B_{\mathrm{S}} h_{\text {gap }}^{2}
$$

and will accelerate charged particles within the polar gap to relativistic energies. For example, a charged particle with $q=e$ in the polar gap of a pulsar with $P=1 \mathrm{~s}$ $(\Omega=2 \pi \mathrm{rad} / \mathrm{s}), B_{\mathrm{S}}=10^{8} \mathrm{~T}$, and $h_{\text {gap }}=100 \mathrm{~m}$ will gain energy $e \Delta V \sim 10^{13} \mathrm{eV}$. The charged particles being accelerated along the magnetic field lines will emit curvature radiation with photon energies of

$$
E_{p h}=\hbar \omega=\frac{3 \gamma^{3} \hbar c}{2 R_{c}}
$$

where $\hbar$ is the reduced Planck constant, $\omega$ is the angular frequency of the curvature radiation photons, $\gamma$ is the Lorentz factor, and $R_{c}$ is the curvature of the field line the particle of energy $\gamma m c^{2}$ moves along, where $m$ is the particle's rest mass. When $\hbar \omega>2 m c^{2}$, a $\gamma$-ray can produce a primary $e^{+}-e^{-}$pair, $\gamma+B \rightarrow e^{-}+$ $e^{+}+B$, each of which will then be accelerated while within the gap $(e \Delta V$, see 
Equation 1.28) and may produce their own curvature radiation. This curvature radiation can then produce more lower energy $e^{+}-e^{-}$pairs, creating a plasma of secondary particles. The exponential growth of new particles through this process is known as a pair cascade. As shown in Figure 1.3, some of these newly created particles being accelerated away the neutron star surface will escape the polar gap $\left(z>h_{\text {gap }}\right)$, entering the force-free region of the pulsar magnetosphere. Without any forces acting upon them along the magnetic field lines $(\vec{E} \cdot \vec{B}=0)$, these particles will continue to travel along them with constant velocity.

It follows that particles that will not produce secondary pairs will therefore have a frequency less then a characteristic frequency given by Equation 1.29 as $\omega_{c}=3 \gamma^{3} c / 2 R_{c}$. Furthermore, the maximum speed a particle with charge $e$ can gain from crossing the entire polar gap can be calculated from Equation 1.28 as

$$
e \Delta V=\gamma_{\max } m c^{2}=e \Omega B_{\mathrm{S}} h^{2}
$$

or

$$
\gamma_{\max }=\frac{q \Omega B_{\mathrm{S}} h^{2}}{m c^{2}}
$$

For a pulsar with $\Omega=2 \pi \mathrm{rad} / \mathrm{s}, B_{\mathrm{S}}=10^{8} \mathrm{~T}$, and $h_{\text {gap }}=100 \mathrm{~m}$, electrons or positrons accelerated across the entire gap would have $\gamma_{\max }=1.2 \times 10^{7}$. As stated by Ruderman \& Sutherland (1975), for $R_{c} \approx 10^{4} \mathrm{~m}$, the curvature radiation of photons from particles accelerated along half that height, $50 \mathrm{~m}$, would have $E_{p h} \approx$ $800 \mathrm{MeV}$ and frequency $\omega_{c} \approx 10^{23} \mathrm{~Hz}$. Secondary particles have much lower energies, 


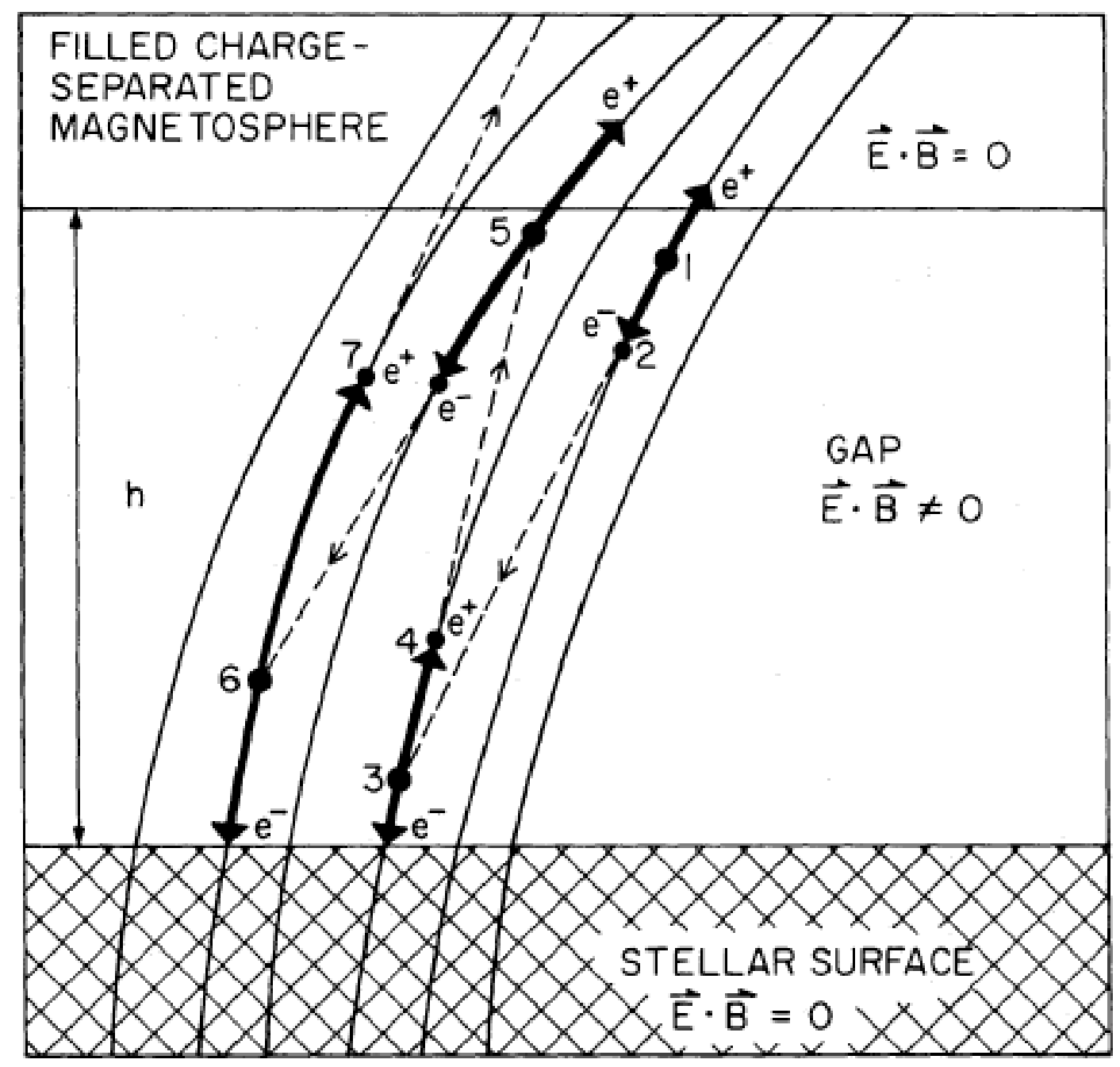

Figure 1.3: Pair cascade within the polar gap occurs when a high-energy particle $\left(\hbar \omega>2 m c^{2}\right)$ produces a curvature radiation photon that produces an $e^{+}-e^{-}$pair at point 1 . In this example, the $e^{+}$enters the force-free region $\left(z>h_{\text {gap }}\right)$ while the $e^{-}$accelerates towards the surface while producing its own curvature radiation photon at point 2. This new photon then also produces its own $e^{+}-e^{-}$pair at point 3. This process continues until the energy of the curvature radiation photons no longer satisfies $\hbar \omega>2 m c^{2}$. (Ruderman \& Sutherland, 1975) 
$\gamma \approx 800$, so for $R_{c} \approx 10^{7} \mathrm{~m}$ the photons would have $E_{p h}<100 \mathrm{MeV}$ and the curvature radiation frequency would be $\omega_{c} / 2 \pi \approx 10^{9} \mathrm{~Hz}$. In other words, highenergy primary particles would produce $\gamma$-ray curvature radiation while low-energy secondary particle curvature radiation would emit radio waves.

Ruderman \& Sutherland (1975) argue that the high brightness temperatures seen in radio emission require coherent emission. The authors suggest that a more energetic (faster) beam of primary particles could catch up with a less energetic beam of secondary particles above the acceleration gap within the force-free region of the pulsar magnetosphere. In this case, the beam would bunch up the secondary particles and enhance their radio-frequency curvature radiation bringing about a higher brightness temperature.

Since the publication of Ruderman \& Sutherland (1975), it has been shown that this analysis cannot explain the ability to both create and maintain the secondary bunching over a long enough time (Melrose, 1992). One method that could explain it better is relativistic plasma emission (Melrose, 1995). In a superstrong magnetic field, the secondary particle plasma is not only highly magnetized, but can be considered one-dimensional along the field lines, where a superstrong magnetic field strength is defined by a critical magnetic field strength,

$$
B_{\text {crit }}=\frac{m_{e}^{2} c^{2}}{e \hbar}=4.4 \times 10^{9} \mathrm{~T}
$$

Note that $B / B_{\text {crit }}$ is then the ratio of the electron cyclotron energy $\left(\hbar e B / m_{e}\right)$ to the rest energy $\left(m_{e} c^{2}\right)$. The particle's motion perpendicular to the magnetic 
field is then lost through synchrotron motion, in this case known as gyromagnetic emission. The lifetime of decay for gyromagnetic emission within the superstrong magnetic field strength regime is very short, $3 / B^{2} \mathrm{~s}$ where $B$ is in Tesla or $\sim 2 \times 10^{-17}\left(B / 0.1 B_{\text {crit }}\right)^{-2} \mathrm{~s}$ in the rest frame of the particle and multiplied by $\gamma$ to convert to the pulsar frame of reference (Melrose, 1995). Therefore, it is considered reasonable to assume a one-dimensional streaming pair plasma. Instabilities within this type of plasma can set up nonlinear processes that can create coherent radiation. However, early estimates of the growth rates were overoptimistic and in reality are too small to allow these instabilities to grow before the particles can escape out of the magnetosphere into the pulsar wind.

Another possible mechanism at work could be maser curvature emission. In the simplest model approximating the magnetic magnetic field lines as circular, the absorption coefficient cannot be negative so it is impossible to create maser emission (Blandford, 1975), but could be possible if curvature drift (Luo \& Melrose, 1992) or field-line distortion (Luo \& Melrose, 1995) is also considered. In order to have a curvature-drift maser, the curvature drift velocity must be non-zero. For a charged particle with mass $m$ and charge $q$ the curvature drift velocity is given by

$$
\vec{v}_{R}=\frac{m v_{\|}^{2}}{q B^{2}} \frac{\vec{R}_{c} \times \vec{B}}{R_{c}^{2}}
$$

where $v_{\|} \equiv \vec{v} \cdot \vec{B} / B$ is the particle's speed along the magnetic field line, and $\vec{R}_{c}$ is the radius of curvature of the magnetic field pointed radially outward. Given this curvature drift, Melrose (1992) shows that the absorption coefficient will be- 
come negative, and hence maser curvature emission is possible, when $\gamma \geq 10^{4}$ and $B>10^{4} \mathrm{~T}$. A drawback of this model, as can be seen in Equation 1.33, is that the curvature drift of electrons and positrons will be in opposite directions which will cause absorption coefficients of the two particle populations to oppose each other and therefore create a net difference between the two particle distributions. Including curvature drift as means to allow the absorption coefficient of a charged particle population to be negative therefore also requires there to be an imbalance between the two charged particle distributions so that one can overcome the other. Conversely, a curvature emission maser may also occur due to a twist of the magnetic field lines and has the advantages of (a) not being sensitive to the magnetic field strength and (b) both electrons and positrons contributing the same sign absorption; this torsion model becomes feasible when $\gamma \geq 40$ (Luo \& Melrose, 1995). It is therefore possible to attain maser curvature emission through either curvature drift or field-line distortion.

\subsubsection{High-Energy Emission}

High energy pulsar emission (X-ray and $\gamma$-ray emission) also involves primary and secondary particles (Daugherty \& Harding, 1982) as well as an acceleration gap as described in Section 1.4.2, but the need for coherent emission is no longer required. The acceleration gap for high energy emission is thought to either be the polar gap region explained in Section 1.4.2 (Daugherty \& Harding, 1986; Harding et al., 2002), or what is known as the outer gap region (Cheng et al., 1986; Chiang 
\& Romani, 1992, 1994; Romani, 1996). The outer gap could explain the wider pulse profiles at high-energies as being due to the emission region being further out in the magnetosphere. It could explain why there is sometimes an observed spin-phase offset between the peaks of the radio and high-energy profiles. Below I will outline how the outer gap differs from the polar gap; for a explanation of the polar gap see Section 1.4.2.

The outer gap, located in the outer magnetosphere, is bounded by the nullcharge surface defined by $\vec{\Omega} \cdot \vec{B}=0$ discussed in Section 1.4.1. Unlike the polar gap, charged particles leaving the outer gap cannot be replenished by the neutron star's surface, therefore leaving large regions of charge depletion between surfaces of opposite charge (see Figure 1.2) creating an electric field. The other borders of this gap are defined by the light cylinder and the last closed field line within the light cylinder, see Figure 1.4.

The potential difference across a gap within the magnetosphere is given by (Cheng et al., 1986)

$$
\Delta V \approx \frac{\Omega^{2} B_{\mathrm{S}} R^{3}}{c} \Delta V \approx 10^{16}\left(\frac{\Omega}{10^{2} \mathrm{~s}^{-1}}\right)^{2}\left(\frac{B_{\mathrm{S}}}{3 \times 10^{8} \mathrm{~T}}\right)\left(\frac{R}{10^{4} \mathrm{~m}}\right)^{3} \mathrm{~V}
$$

Like the polar gap, the large potential difference across the outer gap sustains pair production, $\gamma+B \rightarrow e^{-}+e^{+}+B$, of which each particle which will then be strongly accelerated along the magnetic field lines in opposite directions within the gap. The $\gamma$-rays can be created by either curvature radiation of $e^{-}$and $e^{+}$particles, 


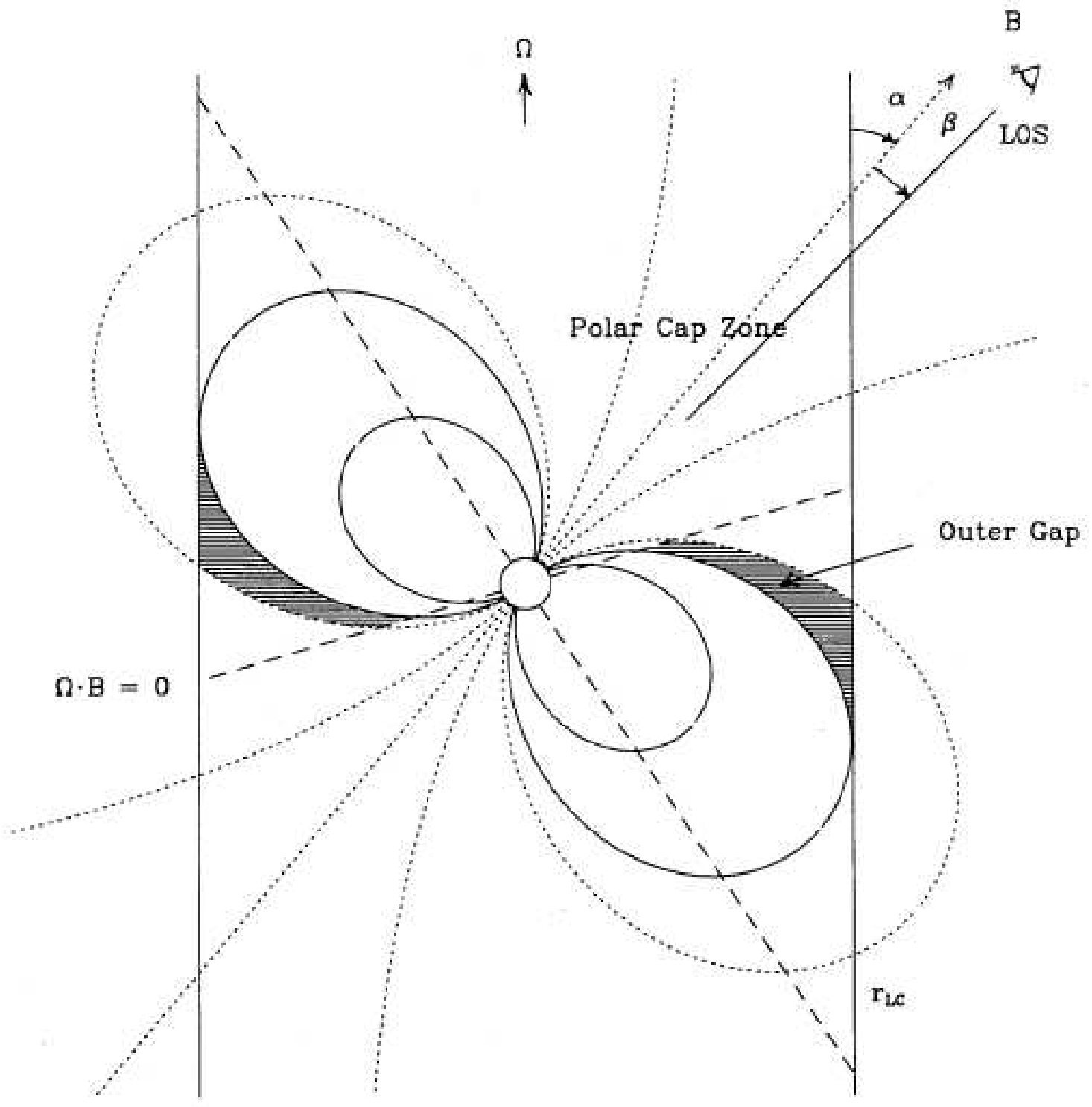

\section{Magnetospheric Gap Geometry}

Figure 1.4: A schematic view of the outer gap emission model, where $\alpha$ is the angle between the dipole magnetic field axis and the rotation axis, and $\beta$ is the impact angle which is the closest approach of the line of sight to the dipole magnetic field axis. The shaded region is the outer gap which is bounded by the null lines (dashed lines), the light cylinder, and the last closed field lines within the light cylinder. (Chiang \& Romani, 1992) 
inverse Compton scattering of relativistic $e^{-}$and $e^{+}$particles, or synchrotron radiation of relativistic $e^{-}$and $e^{+}$particles. In addition to $\gamma$-ray conversions, there can be conversions by collisions with X-rays $\left(\gamma_{x}\right), \gamma+\gamma_{x} \rightarrow e^{-}+e^{+}$, for $\mathrm{GeV} \gamma$-rays. Furthermore, there can also be conversions by collisions with soft (optical or infrared) photons $\left(\gamma_{v}\right), \gamma+\gamma_{v} \rightarrow e^{-}+e^{+}$, for $10^{12} \mathrm{GeV} \gamma$-rays. Regardless of the production method for the secondary particles, they will end up with substantial pitch angle to the magnetic field lines because they are far away from the pulsar, near the light cylinder. With a large part of the secondary particles' velocity perpendicular to the magnetic field lines, they will radiate synchrotron radiation.

Besides the outer gap model, several other similar models have been developed placing the gap in other regions of the pulsar magnetosphere; two examples are the "two pole caustic" (TPC) model (Dyks \& Rudak, 2003) and the "slot gap" (SG) model (Muslimov \& Harding, 2003, 2004). In the TPC model, the acceleration gap extends from the NS surface out to the light cylinder, bound by the last closed field lines in the light cylinder. In the SG model, a tube-like region is bound by the last closed field lines in the light cylinder and a pair formation front (see Figure 1 in Muslimov \& Harding, 2003).

In all the pulsar models discussed thus far, several simplifying assumptions have been made. First, the magnetic field has been modeled as a pure magnetic dipole. Higher-order terms like the quadrupole and octupole vary more quickly with angle and could have a significant effect, especially closer to the surface, greatly affecting the polar gap model. Second, we started off assuming the magnetic field was in a vacuum. In reality, the structure of the magnetosphere would be affected by the 
flow of plasma current density $\vec{J}$, making the force-free condition, Equation 1.21, become $\rho \vec{E}+\vec{J} \times \vec{B}=0$. A realistic time-dependent force-free magnetospheric model cannot be solved analytically, but has been solved numerically (Spitkovsky, 2006). Unfortunately, a force-free magnetosphere lacks any acceleration gaps that explain the observed electromagnetic emission. Nevertheless, it has been shown that it is possible to create the required acceleration gaps in a force-free magnetosphere and hence observed radiation (Bai \& Spitkovsky, 2010); this may require only partial plasma conductivity, meaning the solution lies somewhere between the vacuum and force-free solutions (Harding et al., 2011; Kalapotharakos, Kazanas, Harding \& Contopoulos, 2012; Kalapotharakos, Harding, Kazanas \& Contopoulos, 2012).

To test these emission models and probe within the pulsar magnetosphere, we must compare and contrast X-ray timing observations to radio timing observations. If done simultaneously, a correlation test could lend insight into whether a pulsar's radio and high-energy emission arise from the same mechanism. For example, the peaks of radio and $\gamma$-ray pulse profiles are aligned for the Crab pulsar and PSR B1937+21 (Cheng \& Ruderman, 1977), which suggests both radio and $\gamma$-ray emission may be related. Lundgren et al. (1995) and Bilous et al. (2011) have investigated the correlation between radio giant pulses (see Section 1.5.3) and X-ray as well as $\gamma$-ray emission from the Crab pulsar, but found no correlation. However, Shearer et al. (2003) did find a moderately significant correlation between radio giant pulses and optical emission from the Crab pulsar and was further supported by Collins et al. (2012).

An increase in both non-thermal and thermal X-ray emission close to radio 
pulse detection times should be expected in Rotating Radio Transients (RRATs, see Section 1.5.5) if their sporadicity is due to the reactivation model put forth by Zhang et al. (2007). In the reactivation model, the emission is only active in the magnetosphere when the conditions allow for pair production that instigates coherent radio emission that results in X-ray photons and thermal emission from heating of the polar cap due to backscatter (see Section 1.3). Another model described in Zhang et al. (2007) is radio emission direction reversal, which suggests that RRATs and nulling pulsars (see Section 1.5.5) are always emitting radiation, but the direction of emission reverses direction so that they are emitting their emission away from our line of sight part of the time. In this latter model, there would be no correlation between blackbody X-ray radiation and non-thermal radio and X-ray emission since emission is always active. Therefore synchronous X-ray and radio observations can possibly serve as a differentiator between these different models, which is explored in Chapter 3.

\subsection{A Menagerie of Neutron Stars}

In this section, we will discuss the different categories pulsars are grouped into and will discuss the distinct properties of each: normal pulsars, millisecond pulsars, giant pulsing pulsars, magnetars, nulling pulsars, and RRATs. 


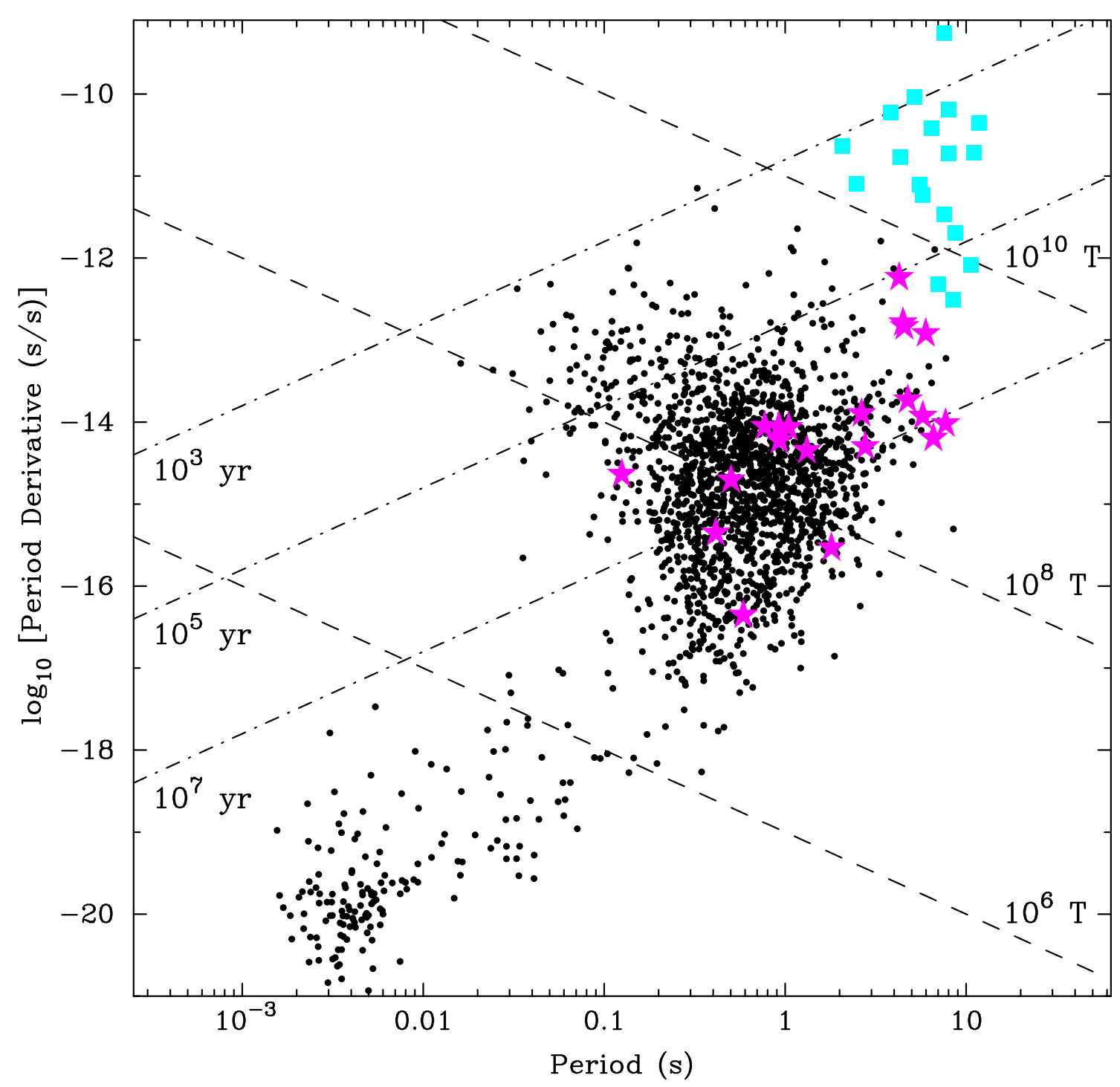

Figure 1.5: The distribution of $P$ and $\dot{P}$ for normal and millisecond pulsars (black dots), magnetars (cyan squares), and RRATs (magenta stars). Lines of constant characteristic age $\tau_{c}$ (dot-dash lines) and constant magnetic field $B_{\mathrm{S}}$ (dashed lines) are shown. 


\subsubsection{Normal Pulsars}

As the name implies, normal pulsars are the most prevalent type of detected pulsar, as can be seen around $P=1 \mathrm{~s}$ in Figure 1.5. Their rotation periods are typically of the order of a second and they have $\dot{P} \sim 10^{-15} \mathrm{~s} / \mathrm{s}$. Referring back to Equations $1.9,1.7$, and 1.2 , we see that they have $B_{\mathrm{S}}$ between $10^{7}$ and $10^{9} \mathrm{~T}$, with $\dot{E}_{\text {rot }}$ between $10^{23}$ and $10^{28} \mathrm{~W}$.

\subsubsection{Millisecond Pulsars}

As can be seen in the bottom-left corner of Figure 1.5, there is a population of neutron stars that are distinct from normal pulsars. This population has shorter rotation periods of a few milliseconds and $\dot{P} \sim 10^{-20} \mathrm{~s} / \mathrm{s}$, and they are therefore termed millisecond pulsars (MSPs). They are typically defined as having $P \lesssim$ $10-30 \mathrm{~ms}$. In this case, $B_{\mathrm{S}}$ is between $10^{4}$ and $10^{5} \mathrm{~T}, \tau_{\mathrm{c}}$ is between $10^{9}$ and $10^{12} \mathrm{yrs}$, and $\dot{E}_{\text {rot }}$ is between $10^{26}$ and $10^{28} \mathrm{~W}$.

The first MSP, PSR B1937+21, was discovered discovered by Backer et al. (1982) and was the fastest spinning pulsar $(P=1.6 \mathrm{~ms})$ until the discovery of PSR J1748-2446ad ( $P=1.4 \mathrm{~ms})$ by Hessels et al. (2006). Of all the MSPs that have been discovered, most are found to be in binary orbits with low-mass companion stars. It is believed that MSPs were once normal pulsars that have since accreted mass from their binary companion, known as recycling (Bhattacharya \& van den Heuvel, 1991). As the companion evolves, its Roche lobe fills, and then overflows and and its matter accretes onto the pulsar. As matter spirals in towards the neutron 


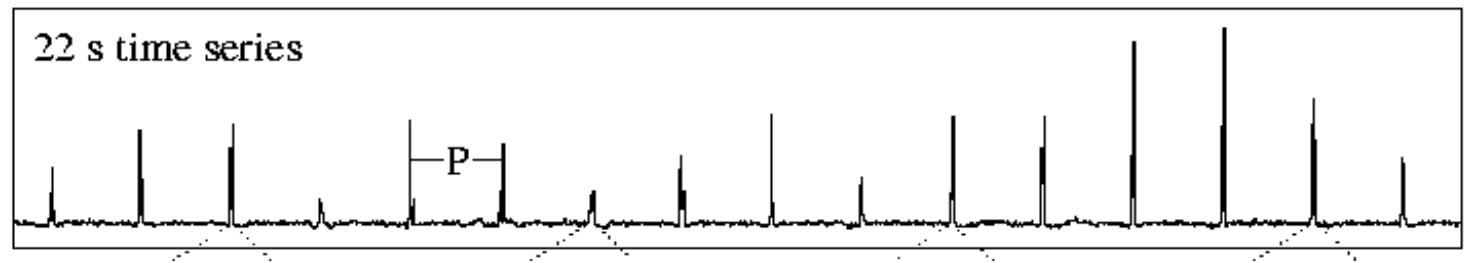

Figure 1.6: Individual pulse amplitude from a pulsar can vary, such as the ones shown here from a $22 \mathrm{~s}$ time series of PSR B0301+19 $(P=1.4 \mathrm{~s})$ observed with the Arecibo radio telescope. The magnitude of the pulse amplitude variation shown here is typical. (Lorimer \& Kramer, 2005)

star, angular momentum is conserved and the rotation of the star speeds up (i.e. $P$ decreases). In addition to speeding up the rotation of the star, the in-falling matter also 'buries' the magnetic field, decreasing $B_{\mathrm{S}}$ (Bisnovatyi-Kogan \& Komberg, 1974; Shibazaki et al., 1989).

\subsubsection{Giant Pulsing Pulsars}

While the time-averaged pulse profile of most pulsars is quite stable, the intensity of individual pulses can vary greatly from pulse to pulse see Figure 1.6. In some cases, pulses can vary in emission strength by several orders of magnitude. Pulsars that emit some single pulses that are $\sim 1000$ times stronger than their time-averaged profile are known as giant-pulsing pulsars. Pulses that emit such strong pulses are quite uncommon for pulsars (Johnston \& Romani, 2002), the first two pulsars to have such strong detected pulses were the Crab pulsar (Lundgren et al., 1995) and the first discovered MSP, PSR B1937+21 (Cognard et al., 1996). It was thought these were the only two pulsars to emit giant pulses for over 20 years, but giant pulses have been observed in a handful of other pulsars over the last decade or so (see Knight, 2006; Knight et al., 2006). 
Unlike normal pulsars whose pulse amplitudes follow a log-normal distribution (e.g. Cairns et al., 2001; Kramer et al., 2002; Cairns et al., 2004; Johnston, 2004), giant-pulsing pulsars have a power-law distribution, or a log-normal distribution with a high-energy power-law tail (Argyle \& Gower, 1972; Lundgren et al., 1995; Johnston \& Romani, 2002; Kramer et al., 2002, 2003; Knight et al., 2006; Popov \& Stappers, 2007; Mickaliger et al., 2012). Giant pulses may therefore arise from a different emission mechanism than normal pulses. It is for this reason Knight et al. (2006) suggest that giant pulses should be defined by short-timescale, narrow-phase emission with power-law statistics.

It has been shown that the giant pulses of the Crab pulsar actually consist of nanopulses which switch between two highly-polarized states (Hankins et al., 2003). The extremely high brightness temperatures of these nanopulses $\left(\sim 10^{37} \mathrm{~K}\right)$ combined with their extremely short timescales suggests that they originate from the coherent emission from a plasma region of a length scale less than one meter within the magnetosphere. It has also been suggested that the giant pulse emission originates from plasma cyclotron resonances (Machabeli \& Usov, 1979; Lyutikov et al., 1999) or magnetic reconnection within the magnetosphere (Lyutikov, 2007). Finally, Knight (2006) shows a correlation between pulsars that emit giant pulses with higher magnetic field values at the light cylinder and $\dot{E}_{\text {rot }}$; the RRAT (see Section 1.5.5) PSR J1819-1458 does not follow this trend. 


\subsubsection{Magnetars}

Magnetars possess the most extreme magnetic field strength of the neutron stars and are in fact the strongest magnetic objects in the known universe with $B_{\mathrm{S}} \sim$ $10^{11} \mathrm{~T}$ (Duncan \& Thompson, 1992). Unlike other neutron stars, the electromagnetic radiation of magnetar emission cannot be powered by rotation nor accretion, but by the decay of their strong magnetic fields. Most magnetars are detected only in the $\gamma$ - and X-ray wavelengths, while four magnetars, XTE J1810-197, 1E 1547.0-5408, PSR J1622-4950, and SGR J1745-2900 also have detected radio pulses (Camilo et al., 2006, 2007; Levin et al., 2010; Rea et al., 2013). One of these magnetars, PSR J1622-4950, is the first magnetar to be discovered via its radio emission, the X-ray source coincident with PSR J1622-4950 is in X-ray quiescence (Levin et al., 2010). If a forming neutron star has the proper temperature, magnetic field, and spin, then it is possible that a magnetohydrodynamic dynamo process can convert some of the heat and rotational energy into additional magnetic energy, enhancing the supernova remnant's magnetic field strength. The likelihood of a magnetar forming from a supernova explosion through this process rather than a normal pulsar is estimated to be $8-9 \%$ (Popov \& Prokhorov, 2006). The magnetars are shown in the upper-right corner of Figure 1.5.

Long-term timing of pulsars has revealed what are known as glitches among some young normal pulsars and magnetars (Lyne et al., 1996; Lyne et al., 2009). A glitch is a discontinuity in $\dot{P}$, where it is observed that the pulsar suddenly speeds up its rotation. Glitches in young pulsars are caused by a 'stellar quake' where the 
outer crust of the neutron star surface gains angular momentum from the quickly rotating superfluid inside the neutron star, whereas a glitch in a magnetar is due to a magnetospheric reconfiguration. Changes in both pulse profiles and brightness have been observed to accompany glitches in magnetars (Woods et al., 2001, 2004; Muno et al., 2007; Woods et al., 2007). There is also one case, magnetar 1E 2259+586, where long-term timing has revealed an 'anti-glitch', in which the magnetar suddenly slowed down (Archibald et al., 2013).

\subsubsection{Nulling Pulsars and Rotating Radio Transients}

Nulling pulsars and RRATs are like other pulsars which are typified by their repeating and dispersed pulses, but unlike other pulsars, a pulse is not detected for every rotational period. While pulsars were originally found by searching for the emission of single pulses (Hewish et al., 1968), searches are now prominently periodicity-based using Fourier techniques and have the advantage of higher sensitivity to pulsars' repeating signals. Among the many pulsars found in periodicitybased searches, it has been found that a fraction of these neutron stars cease emitting detectable pulses part of the time; this process is dubbed pulse nulling and has so far been detected in over 100 pulsars (Backer, 1970; Hesse \& Wielebinski, 1974; Ritchings, 1976; Biggs, 1992; Wang et al., 2007). Many more pulsars may null, but the nulling may not be detectable because the pulsars' weaker pulses fall below the sensitivity of current telescopes.

RRATs exhibit more extreme pulse nulling with the time between detected 
pulses ranging from a single rotation period (seconds) to hours and beyond. RRATs are undetectable using periodicity-based searches and have only recently been discovered through a single pulse search of archival data from the 1.4-GHz Parkes Multibeam Pulsar Survey (PMPS) by McLaughlin et al. (2006). Of the more than 2000 known pulsars, roughly 180 have now been identified as RATTs $^{1}$ (Hessels et al., 2008; Burke-Spolaor \& Bailes, 2010; Deneva et al., 2009; Keane et al., 2010, 2011). Single pulses are usually sporadically detected, but have similar widths and intensities to the individual pulses of other pulsars. Even though the rotational period of RRATs cannot be determined using Fourier techniques, their underlying periodicity can be found by determining the greatest common divisor of every interval between the pulses for each RRAT. While this technically only yields an upper limit to the rotational period which could be an integer multiple of the RRAT's true rotational period, it is statistically unlikely that that is the case once many pulse intervals are included. RRATs have rotational periods around a second, about the same order of magnitude as normal pulsars, and $\dot{P}$ values ranging from normal pulsar values up to near magnetar values (Burgay et al., 2007).

There appears to be a spectrum of nulling pulsar timescales that bridges RRATs with other pulsars ranging from "standard" nulling pulsars up to where the emission timescale appears to be less than one rotational period, i. e. some RRATs, see Figure 1.7 (Burke-Spolaor, 2013). For a working definition, RRATs can be defined as pulsars that were discovered only through their single pulses; this definition is telescope dependent. Like all pulsars, detected individual pulses vary in intensity

\footnotetext{
${ }^{1}$ http://astro.phys.wvu.edu/rratalog/
} 

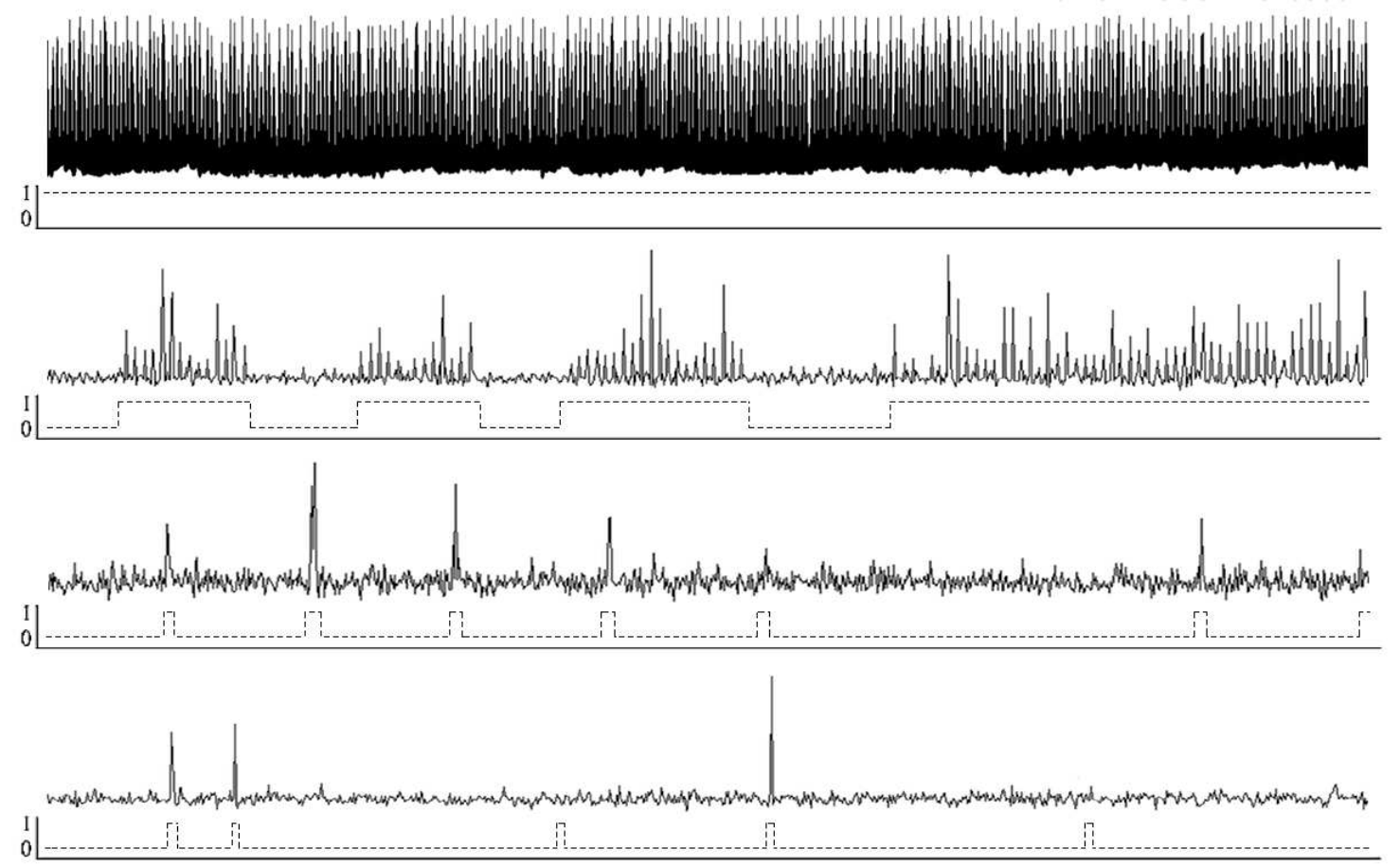

Figure 1.7: Dedispersed time series showing a range of emission activity timescales from different pulsars (top to bottom: Vela, PSRs J1646-6831, J1647-36, and J1226-32; archival data from Edwards et al. (2001), all panels are of equal time duration). The binary plot below each time series illustrates the null/emission state of the pulsar. PSR J1647-36 displays $\sim 5-10$ pulse emission clusters, while PSR J1226-32 shows single pulses that may suggest an emission timescale less than one rotational period. (Burke-Spolaor, 2013)

from pulse to pulse. For some RRATs, a telescope may not have the sensitivity to detect the weaker pulses, but only the stronger pulses. Weltevrede et al. (2006) proposed that RRAT emission may be similar to that of nearby PSR B0656+14 and only appears different because RRATs are farther from the Earth. Other RRATs, however, may not be emitting any pulses in between the pulses we detect. One interpretation of this scenario is that RRATs could be re-activated dead pulsars that have conditions conducive to $e^{-}-e^{+}$pair production only part of the time (Zhang et al., 2007).

McLaughlin et al. (2009) have shown that RRATs have longer periods and 
magnetic fields than other pulsars, although Cordes \& McLaughlin (2003) show that pulsars with longer periods are more likely to have a higher SNR in singlepulse searches, so this may be a selection effect. Lyne et al. (2009) have detected two 'anti-glitches' in PSR J1819-1458, suggesting that it may be an exhausted magnetar (see Section 1.5.4). Knight et al. (2006) have suggested that RRATs may be related to giant-pulsing pulsars (Section 1.5.3). Modulation of the emitted pulses from a radiation belt, similar to a planetary magnetospheres (Luo \& Melrose, 2007), or from disturbances from an asteroid belt (Li, 2006; Cordes \& Shannon, 2008), can also explain the transient detection of pulses from RRATs.

\subsection{Outline of Dissertation}

The goal of this dissertation is to characterize the multiwavelength emission properties of RRATs. In Chapter 2, the statistics of many radio observations are studied. In Chapter 3, the simultaneous detection of both radio and X-ray radiation originating from one particular RRAT, PSR J1819-1458, is studied in order to help characterize the emission of pulses at both wavelengths. The dissertation is summarized in Chapter 4. 


\section{Chapter 2}

\section{RRAT Pulse Amplitude Distributions and Spectral Indices}

\subsection{Introduction}

Two of the parameters that can help characterize emission mechanisms are pulse amplitude distributions and spectral indices. Pulse amplitude distributions are also important for determining a luminosity function which can constrain the birth rate of Galactic RRATs and, if they are evolutionarily related, Galactic pulsars. This is crucial for estimations of the total number of neutron stars in the Galaxy and reconciling this number with the Galactic supernova rate.

In cases where single pulse amplitude distributions have been studied (e.g.

Cairns et al., 2001; Kramer et al., 2002; Cairns et al., 2004; Johnston, 2004), they typically have a probability distribution function (PDF) that can be described by a log-normal function,

$$
\operatorname{PDF}(S)=\frac{1}{S \sigma_{\ln } \sqrt{2 \pi}} \exp \left(-\frac{\left(\ln S-\mu_{\ln }\right)^{2}}{2 \sigma_{\ln }^{2}}\right)
$$

where $S$ is the flux density, $\mu_{\ln }$ is the mean of the natural logarithm of the flux density, and $\sigma_{\ln }$ is the standard deviation of the natural logarithm of the flux density. Conversely, giant-pulsing pulsars (Knight et al., 2006) seem to have radio pulse 
amplitude distributions that resemble a power-law

$$
\operatorname{PDF}(S) \propto S^{-K}
$$

where $K$ is a constant. It is even possible to have a combination of these two distributions, e.g. Kramer et al. (2003) studied the flux density distributions of PSR B1133+16, which at most frequencies had a log-normal distribution, but a power-law component due to giant pulses became apparent at higher frequencies. Finally, Serylak et al. (2009) has recently shown that for at least one magnetar, XTE J1810-197, the single pulse amplitude distributions vary from log-normal and power-law distributions at different frequencies and at different epochs. It is then worthwhile to study the pulse amplitude distributions of RRATs to see if they resemble those of normal pulsars, giant-pulsing pulsars, magnetars, or if they are altogether different.

Likewise, studying how the flux density changes with frequency can provide further insight. For a typical pulsar, the mean flux density follows a power-law distribution with radio frequency, $\langle S\rangle \propto f^{\alpha_{\mathrm{SI}}}$, where $f$ is the frequency of emission and $\alpha_{\mathrm{SI}}$ is the spectral index. The mean value of $\alpha_{\mathrm{SI}}$ for radio pulsars given by Maron et al. (2000) is $\sim-1.8$, with values in the range $-4.0 \lesssim \alpha_{\mathrm{SI}} \lesssim-0.3$. The four magnetars with detected radio emission, XTE J1810-197, 1E 1547.0-5408, PSR J1622-4950, and SGR J1745-2900, the radio spectrum appears to be flat or possibly even inverted, $\alpha_{\mathrm{SI}}>-1$ (Camilo et al., 2006, 2007, 2008; Lazaridis et al., 2008; Levin et al., 2010; Rea et al., 2013). 
In this chapter, we will characterize both the pulse amplitude distributions and spectral indices of RRATs to see how they relate to normal pulsars, magnetars, and giant-pulsing pulsars. In Section 2.2, we describe the observations used for our analysis. In Section 2.3 we describe the analysis. We discuss how our results compare with similar studies of other neutron stars in Section 2.4.

\subsection{Observations}

The PMPS surveyed the Galactic plane with the 64-m Parkes telescope in NSW, Australia using a 13-beam 1.4-GHz receiver. All eight RRATs discussed in this paper were discovered by re-analysis of the archival data by McLaughlin et al. (2006); their measured and derived properties are given in Table 2.1. Follow-up observations of the RRATs using the Parkes telescope were made at four other frequencies. Table 2.2 lists the observing parameters for each observation frequency. We also list the diffractive scintillation timescales and bandwidths of all eight RRATs at each frequency predicted by the NE2001 Galactic free electron density model of Cordes \& Lazio (2002) with positions and DMs reported in Lyne et al. (2009) for PSR J1819-1458; McLaughlin et al. (2009) for PSRs J0847-4316, J1317-5759, J1444-6026, J1826-1419, J1846-0257, and J1913+1330; and Cui et al. (2013) for PSR J1754-3014. While the predicted scintillation timescales are on the order of the periods for all of these RRATs, the predicted diffractive scintillation bandwidths are all less than $1 \mathrm{MHz}$, much less than the observation bandwidths listed in Table 2.2. This implies that it is likely that the short-term flux density variations are entirely 
Table 2.1: Spin-Down Properties for Eight RRATs.

\begin{tabular}{lllllll}
\hline Name & $\begin{array}{l}P \\
(\mathrm{~s})\end{array}$ & $\begin{array}{l}\mathrm{DM} \\
\left(\mathrm{pc} \mathrm{cm}{ }^{-3}\right)\end{array}$ & $\begin{array}{l}d \\
(\mathrm{kpc})\end{array}$ & $\begin{array}{l}\log \left[\tau_{c}\right] \\
(\log [\mathrm{yr}])\end{array}$ & $\begin{array}{l}\log [B] \\
(\log [\mathrm{T}])\end{array}$ & $\begin{array}{l}\log \left[\dot{E}_{\mathrm{rot}}\right] \\
(\mathrm{W})\end{array}$ \\
\hline $\mathrm{J} 0847-4316$ & 5.98 & 292.5 & 3.4 & 5.9 & 9.4 & 24.3 \\
$\mathrm{~J} 1317-5759$ & 2.64 & 145.3 & 3.0 & 6.5 & 8.8 & 24.4 \\
$\mathrm{~J} 1444-6026$ & 4.76 & 367.7 & 5.5 & 6.6 & 9.0 & 23.8 \\
$\mathrm{~J} 1754-3014$ & 1.32 & 293 & 2.2 & 6.7 & 8.4 & 24.9 \\
$\mathrm{~J} 1819-1458$ & 4.26 & 196.0 & 3.6 & 5.1 & 9.7 & 25.4 \\
$\mathrm{~J} 1826-1419$ & 0.771 & 160 & 3.2 & 6.1 & 8.4 & 25.9 \\
$\mathrm{~J} 1846-0257$ & 4.48 & 237 & 5.2 & 5.6 & 9.4 & 24.8 \\
$\mathrm{~J} 1913+1330$ & 0.923 & 175.64 & 5.7 & 6.2 & 8.4 & 25.6 \\
\hline
\end{tabular}

For each RRAT, we give periods, dispersion measures, distances, and base-10 logarithms of the derived parameters characteristic age, surface dipole magnetic field strength, and rotational energy loss rate (see McLaughlin et al., 2009; Cui et al., 2013).

due to intrinsic variations in the RRATs' emission. It does not, however, rule out refractive scintillation as the source of long-term flux density variations.

Observations of many RRATs were also made using the Robert C. Byrd Green Bank Telescope (GBT) in West Virginia, as well as the Arecibo Telescope in Puerto Rico. These observations are not included here due to the systematic errors arising from comparing flux densities measured with different observing systems. The derived spectra were similar but given the smaller numbers of pulses detected with the Green Bank Telescope and Arecibo, the errors are much larger than those reported here for Parkes data. Finally, analysis of PSRs J1839-01, J1848-12, or J1911+00 from McLaughlin et al. (2006) is not included here because of the small number of pulses detected so far due to their low burst rates. 
Table 2.2: Observing Parameters for Parkes Observations

\begin{tabular}{|c|c|c|c|c|c|c|c|c|c|c|}
\hline $\begin{array}{c}f \\
(\mathrm{MHz})\end{array}$ & $\begin{array}{c}\Delta f \\
(\mathrm{MHz})\end{array}$ & $N_{\text {chan }}$ & $\begin{array}{c}G \\
(\mathrm{~K} / \mathrm{Jy})\end{array}$ & RRAT & $\begin{array}{c}t_{\text {samp }} \\
(\mathrm{ms})\end{array}$ & $\begin{array}{l}T_{\text {sys }} \\
(\mathrm{K})\end{array}$ & $\begin{array}{c}\sigma_{\mathrm{RN}} \\
(\mathrm{mJy})\end{array}$ & $\begin{array}{c}\langle S\rangle_{\min } \\
(\mathrm{mJy})\end{array}$ & $\begin{array}{c}\Delta t_{\text {DISS }} \\
(\mathrm{s})\end{array}$ & $\begin{array}{l}\Delta f_{\text {DISS }} \\
(\mathrm{kHz})\end{array}$ \\
\hline \multirow[t]{3}{*}{685} & 64 & 256 & 0.59 & $\mathrm{~J} 1317-5759$ & 5.161 & 57 & 150 & 1.8 & 24.3 & 2.5 \\
\hline & & & & $\mathrm{J} 1819-1458$ & 8.326 & 135 & 278 & 3.3 & 9.8 & 0.4 \\
\hline & & & & $\mathrm{J} 1913+1330$ & 1.804 & 81 & 357 & 4.2 & 24.7 & 1.4 \\
\hline \multirow[t]{2}{*}{1373} & 288 & 96 & 0.67 & J0847-4316 & 11.675 & 28 & 20 & 0.2 & 13.1 & 1.4 \\
\hline & & & & J1819-1458 & 8.326 & 39 & 34 & 0.4 & 13.5 & 1.4 \\
\hline \multirow[t]{8}{*}{1390} & 256 & 512 & 0.67 & J0847-4316 & 11.675 & 28 & 22 & 0.3 & 13.2 & 1.5 \\
\hline & & & & J1317-5759 & 5.161 & 26 & 30 & 0.4 & 33.5 & 10. \\
\hline & & & & $\mathrm{J} 1444-6026$ & 9.294 & 31 & 26 & 0.3 & 12.7 & 0.8 \\
\hline & & & & J1754-3014 & 2.539 & 37 & 60 & 0.7 & 45.7 & 30. \\
\hline & & & & J1819-1458 & 8.326 & 39 & 35 & 0.4 & 13.6 & 1.5 \\
\hline & & & & J1826-1419 & 1.505 & 36 & 77 & 0.9 & 22.5 & 4.5 \\
\hline & & & & $\mathrm{J} 1846-0257$ & 8.744 & 36 & 32 & 0.4 & 10.8 & 0.6 \\
\hline & & & & $\mathrm{J} 1913+1330$ & 1.804 & 30 & 59 & 0.7 & 34.0 & 5.8 \\
\hline \multirow[t]{6}{*}{1517} & 576 & 192 & 0.67 & J0847-4316 & 11.675 & 32 & 16 & 0.2 & 13.8 & 2.1 \\
\hline & & & & J1317-5759 & 5.161 & 30 & 23 & 0.3 & 34.8 & 20. \\
\hline & & & & J1444-6026 & 9.294 & 34 & 19 & 0.2 & 13.3 & 1.2 \\
\hline & & & & J1754-3014 & 2.539 & 38 & 42 & 0.5 & 47.5 & 40. \\
\hline & & & & J1819-1458 & 8.326 & 40 & 24 & 0.3 & 14.1 & 2.1 \\
\hline & & & & J1826-1419 & 1.505 & 38 & 54 & 0.6 & 23.4 & 6.3 \\
\hline 3029 & 768 & 256 & 0.67 & J1819-1458 & 8.326 & 32 & 17 & 0.2 & 19.3 & 490. \\
\hline
\end{tabular}

Center frequency $f$, bandwidth $\Delta f$, number of frequency channels $N_{\text {chan }}$, and gain $G$. For each RRAT we provide the sampling time after rebinning $t_{\mathrm{samp}}=P / 512$, the system temperature $T_{\text {sys }}$, the radiometer noise $\sigma_{\mathrm{RN}}$ calculated using Equation 2.3 with $\beta_{\text {dig }}=\sqrt{\pi / 2}$ and $N_{\text {pol }}=2$, and the minimum detectable mean flux density of a single pulse $\langle S\rangle_{\min }$. Diffractive scintillation times $\Delta t_{\text {DISS }}$, assuming a $100 \mathrm{~km} / \mathrm{s}$ source velocity, and diffractive scintillation bandwidths $\Delta f_{\text {DISS }}$ from Cordes \& Lazio (2002), are provided for comparison. 


\subsection{Analysis}

In this section, we describe our methods for calculating flux densities of single pulses, deriving mean flux densities from fitting the single pulse flux density distributions, and calculating spectral indices for each RRAT, with careful attention to possible sources of error and the underlying assumptions.

\subsubsection{Flux Calculations}

For normal pulsars, flux densities are calculated by first averaging the signal over many periods of the pulsar, which increases the signal-to-noise ratio $(\mathrm{S} / \mathrm{N})$. For the RRATs, we must calculate the flux density of each single pulse detected (averaged over one pulse period) and then calculate the mean flux density of all the detected pulses. We do not average in the zero flux density values of spin periods where no pulse is detected; the mean flux densities quoted here represent only the mean of times when RRAT pulses are detected. Pulses were first searched for by dedispersing filterbank data both at the DM of the RRAT and with no dispersion. Pulses with amplitudes greater than six times the rms noise level of the rebinned time series were recorded. The SIGPROC ${ }^{1}$ pulsar processing package was used for the dedispersion and the pulse searching. These pulses were then folded at the known period of the RRAT using 512 bins across the pulse period as in McLaughlin et al. (2009). The pulses were fit to the solutions presented in McLaughlin et al. (2009) and pulses whose arrival times did not fit the model (to within the RMS timing

\footnotetext{
${ }^{1} \mathrm{http}: / /$ sigproc.sourceforge.net
} 
precision of order milliseconds, roughly the width of the pulses) were excluded. Epochs for which the RRATs were not detected at all are still included for the purposes of calculating the detection rates. The number of pulses detected at each frequency $N_{\mathrm{p}}$, the number of epochs observed at each frequency $N_{\mathrm{e}}$, and the rate of pulse detection are given in Table 2.3. The time resolution will be degraded due to dispersion and interstellar scattering. However, at these frequencies and spin periods, these effects are not important, making the sampling time, $P / 512$, only very slightly less than the effective resolution of our profiles.

In order to calculate flux densities, a region including $2 \%$ of the rotation period around the pulse peak and the remaining $98 \%$ were defined as on- and off-pulse regions, respectively. The individual pulses of every RRAT except J1754-3014 had a duty cycle greater than $2 \%$, the width of four of the 47 pulses from J1754-3014 exceeded $0.02 P$. Flux densities were then calculated by subtracting the off-pulse mean from the data and then scaling the off-pulse standard deviation to the radiometer noise

$$
\sigma_{\mathrm{RN}}=\frac{\beta_{\mathrm{dig}} T_{\mathrm{sys}}}{G \sqrt{N_{\mathrm{pol}} t_{\mathrm{samp}} \Delta f}}
$$

where $\beta_{\text {dig }}$ is a correction factor accounting for the loss in sensitivity due to digitization, $T_{\text {sys }}$ is the system temperature, $G$ is the telescope gain, $N_{\text {pol }}$ is the number of polarizations summed, $t_{\mathrm{samp}}$ is the sampling time of one bin, and $\Delta f$ is the bandwidth of the observation. In this analysis, we included a $10 \%$ uncertainty in both $T_{\text {sys }}$ and $G$ to account for systematic errors.

The brightest pulses detected in 1.4-GHz observations for each RRAT are 
Table 2.3: Flux Density Statistics

\begin{tabular}{|c|c|c|c|c|c|c|c|c|c|c|c|c|c|c|c|}
\hline Name & $\begin{array}{r}f \\
(\mathrm{MHz})\end{array}$ & $N_{\mathrm{e}}$ & $N_{\mathrm{p}}$ & $\begin{array}{r}\text { Rate } \\
\left(\mathrm{hr}^{-1}\right)\end{array}$ & $\begin{array}{l}\langle S\rangle \\
(\mathrm{mJy})\end{array}$ & $\begin{array}{l}\sigma_{S} \\
(\mathrm{mJy})\end{array}$ & $\begin{array}{l}\langle w\rangle \\
(\mathrm{ms})\end{array}$ & $\begin{array}{l}\sigma_{w} \\
(\mathrm{~ms})\end{array}$ & $\alpha_{\mathrm{SI}}$ & $\kappa_{\mathrm{SI}}$ & $\begin{array}{l}\langle S\rangle_{\ln } \\
(\mathrm{mJy})\end{array}$ & $\begin{array}{l}\sigma_{S \ln } \\
(\mathrm{mJy})\end{array}$ & $Q_{\mathrm{KS}}$ & $\alpha_{\mathrm{SIln}}$ & $\kappa_{\mathrm{SI} \ln }$ \\
\hline \multirow[t]{3}{*}{ J0847-4316 } & 1373 & 2 & 5 & 4.3 & $0.7(2)$ & 0.2 & $50(20)$ & 40 & \multirow[t]{3}{*}{$-2.87(9)$} & \multirow[t]{3}{*}{0.06} & $0.74(1)$ & $0.23(2)$ & 0.90 & \multirow[t]{3}{*}{$-2.5(3)$} & \multirow[t]{3}{*}{1.38} \\
\hline & 1390 & 63 & 132 & 2.7 & $0.73(3)$ & 0.25 & $30(2)$ & 13 & & & $0.736(6)$ & $0.23(1)$ & 0.76 & & \\
\hline & 1517 & 8 & 22 & 3.1 & $0.57(6)$ & 0.20 & $28(5)$ & 9 & & & $0.59(1)$ & $0.22(2)$ & 0.91 & & \\
\hline \multirow[t]{3}{*}{ J1317-5759 } & 685 & 6 & 21 & 2.3 & $9.6(8)$ & 3.2 & $17(2)$ & 4 & \multirow[t]{3}{*}{$-2.35(3)$} & \multirow[t]{3}{*}{0.26} & $10.0(1)$ & $4.1(2)$ & 0.83 & \multirow[t]{3}{*}{$-2.40(2)$} & \multirow[t]{3}{*}{0.74} \\
\hline & 1390 & 90 & 245 & 5.0 & $1.80(6)$ & 1.05 & $12.2(5)$ & 4.5 & & & $1.82(4)$ & $1.13(7)$ & 0.96 & & \\
\hline & 1517 & 7 & 27 & 7.7 & $1.5(2)$ & 0.8 & $13(2)$ & 4 & & & $1.51(5)$ & $0.73(8)$ & 0.93 & & \\
\hline \multirow[t]{2}{*}{ J1444-6026 } & 1390 & 73 & 40 & 0.5 & $1.1(1)$ & 0.3 & $20(2)$ & 4 & \multirow[t]{2}{*}{$-7(4)$} & \multirow[t]{2}{*}{-} & $1.096(9)$ & $0.36(2)$ & 0.91 & \multirow[t]{2}{*}{$-6.6(2)$} & \multirow[t]{2}{*}{-} \\
\hline & 1517 & 9 & 4 & 0.5 & $0.6(2)$ & 0.1 & $18(7)$ & 3 & & & $0.62(1)$ & $0.12(2)$ & 0.97 & & \\
\hline \multirow[t]{2}{*}{ J1754-3014 } & 1390 & 49 & 38 & 0.6 & $2.2(1)$ & 1.3 & $7.0(7)$ & 2.7 & \multirow[t]{2}{*}{$-5(2)$} & \multirow[t]{2}{*}{-} & $2.23(4)$ & $1.48(8)$ & 0.98 & \multirow[t]{2}{*}{$-5.3(3)$} & \multirow[t]{2}{*}{-} \\
\hline & 1517 & 6 & 9 & 1.6 & $1.4(2)$ & 0.5 & $8(2)$ & 1 & & & $1.40(2)$ & $0.42(4)$ & 0.68 & & \\
\hline \multirow[t]{5}{*}{ J1819-1458 } & 685 & 4 & 10 & 4.0 & $9(2)$ & 5 & $12(4)$ & 4 & \multirow[t]{5}{*}{$-1.3(3)$} & \multirow[t]{5}{*}{1.84} & $8.0(1)$ & $3.5(2)$ & 0.77 & \multirow[t]{5}{*}{$-2.0(3)$} & \multirow[t]{5}{*}{10.3} \\
\hline & 1373 & 2 & 10 & 9.7 & $1.5(3)$ & 0.5 & $15(6)$ & 4 & & & $1.52(5)$ & $0.54(9)$ & 0.97 & & \\
\hline & 1390 & 72 & 1074 & 23.9 & $1.64(4)$ & 1.21 & $12.1(3)$ & 5.4 & & & $1.54(3)$ & $0.96(5)$ & 0.06 & & \\
\hline & 1517 & 5 & 83 & 33.2 & $1.6(1)$ & 1.0 & $15(1)$ & 5 & & & $1.52(4)$ & $0.84(8)$ & 0.98 & & \\
\hline & 3029 & 4 & 24 & 9.6 & $0.70(9)$ & 0.29 & $16(3)$ & 4 & & & $0.73(3)$ & $0.31(6)$ & 0.68 & & \\
\hline \multirow[t]{2}{*}{ J1826-1419 } & 1390 & 56 & 47 & 0.8 & $3.8(2)$ & 1.5 & $4.5(4)$ & 1.2 & \multirow[t]{2}{*}{$-3(1)$} & \multirow[t]{2}{*}{-} & $3.85(4)$ & $1.59(6)$ & 0.99 & \multirow[t]{2}{*}{$-1.4(3)$} & \multirow[t]{2}{*}{ - } \\
\hline & 1517 & 7 & 10 & 1.4 & $3.0(3)$ & 1.6 & $6(1)$ & 2 & & & $3.42(9)$ & $2.4(2)$ & 0.86 & & \\
\hline J1846-0257 & 1390 & 45 & 35 & 0.9 & $0.80(9)$ & 0.25 & $15(2)$ & 5 & - & - & $0.812(7)$ & $0.27(1)$ & 0.91 & - & - \\
\hline \multirow[t]{2}{*}{$\mathrm{J} 1913+1330$} & 685 & 5 & 28 & 3.1 & $9.4(9)$ & 2.9 & $3.5(4)$ & 0.8 & $-1.6(1)$ & - & $9.76(9)$ & $3.5(2)$ & 0.65 & $-1.72(2)$ & - \\
\hline & 1390 & 29 & 140 & 7.0 & $3.0(1)$ & 1.6 & $5.0(2)$ & 1.4 & & & $2.90(3)$ & $1.22(6)$ & 0.72 & & \\
\hline
\end{tabular}

For each RRAT, we give the center frequencies $f$, number of epochs observed at that frequency $N_{\mathrm{e}}$, number of pulses detected at that frequency $N_{\mathrm{p}}$, rate of pulse detection, mean flux density $\langle S\rangle$, standard deviation of the mean flux density $\sigma_{S}$, mean pulse width $\langle w\rangle$, standard deviation of the pulse widths $\sigma_{w}$, spectral index $\alpha_{\mathrm{SI}}$ based on $\langle S\rangle$, the error scaling factor $\kappa_{\mathrm{SI}}$ so that $\chi_{1}^{2}=1$, the mean flux densities $\langle S\rangle_{\ln }$ and standard deviations $\sigma_{S \ln }$ derived from fitting lognormal flux density distributions, the significance level $Q_{\mathrm{KS}}$ of the Kolmogorov-Smirnov goodness-to-fit statistic, spectral index $\alpha_{\mathrm{SI} \text { In }}$ based on $\langle S\rangle_{\ln }$ values, and the error scaling factor $\kappa_{\mathrm{SI} \text { ln }}$ so that $\chi_{2}^{2}=1$. The numbers in parentheses after $\langle S\rangle,\langle W\rangle$, and $\alpha_{\mathrm{SI}}$ are the $1 \sigma$ errors derived from both statistical and systematic errors. The numbers in parentheses after $\langle S\rangle_{\ln }, \sigma_{S \ln }$, and $\alpha_{\mathrm{SI} \text { ln }}$ are the $1 \sigma$ errors derived solely from fitting the log-normal PDF to the data. 
shown in Figure 2.1. In the PMPS, the data were one-bit digitized and orthogonal polarizations were summed (Manchester et al., 2001), and hence $\beta_{\operatorname{dig}}=\sqrt{\pi / 2}$ and $N_{\text {pol }}=2$ (Brinklow, 1989). The system temperature is defined as the sum $T_{\text {sys }}=$ $T_{\text {rec }}+T_{\text {spill }}+T_{\text {atm }}+T_{\text {sky }}$ (see Lorimer \& Kramer, 2005, Appendix 1). For the zenith angles available at the Parkes Telescope $\left(\lesssim 60^{\circ}\right),{ }^{2} T_{\text {spill }} \approx 0$. The emission due to the atmosphere, $T_{\text {atm }}$, only makes a significant contribution to $T_{\mathrm{sys}}$ when observing at frequencies above $5 \mathrm{GHz}$ (Lorimer \& Kramer, 2005) and at large zenith angles. Therefore, we took the system temperature to be the sum of the receiver temperature and the appropriate sky temperature at the observation frequency, scaled from the 408-MHz values of Haslam et al. (1981) assuming a spectral index of -2.6 (Lawson et al., 1987), i.e. $T_{\text {sys }} \approx T_{\text {rec }}+T_{\text {sky }}$. Values for both $T_{\text {rec }}$ and $G$ were taken from the Parkes Users Guide. ${ }^{2}$ Values of $T_{\text {sys }}$ for each RRAT at each observing frequency are provided in Table 2.2 .

Because of the many weak pulses in our data, we found that fitting a smooth Gaussian to the profiles provides a more robust estimation of the flux density than simply integrating the observed profile. Therefore, once the amplitude of the pulse was scaled to flux density units, a Gaussian profile was fit to each pulse profile using a least-squared fitting algorithm. In cases where a second peak was present (at at least the $1 \sigma_{\mathrm{RN}}$ level) after fitting the first peak, a second Gaussian peak was fit. In cases where more than two peaks were present, adding a third peak did not have a noticeable effect on the mean flux density calculations, i.e. the change was much

\footnotetext{
${ }^{2}$ http://www.parkes.atnf.csiro.au/observing/documentation/ user_guide/pks_ug.html
} 

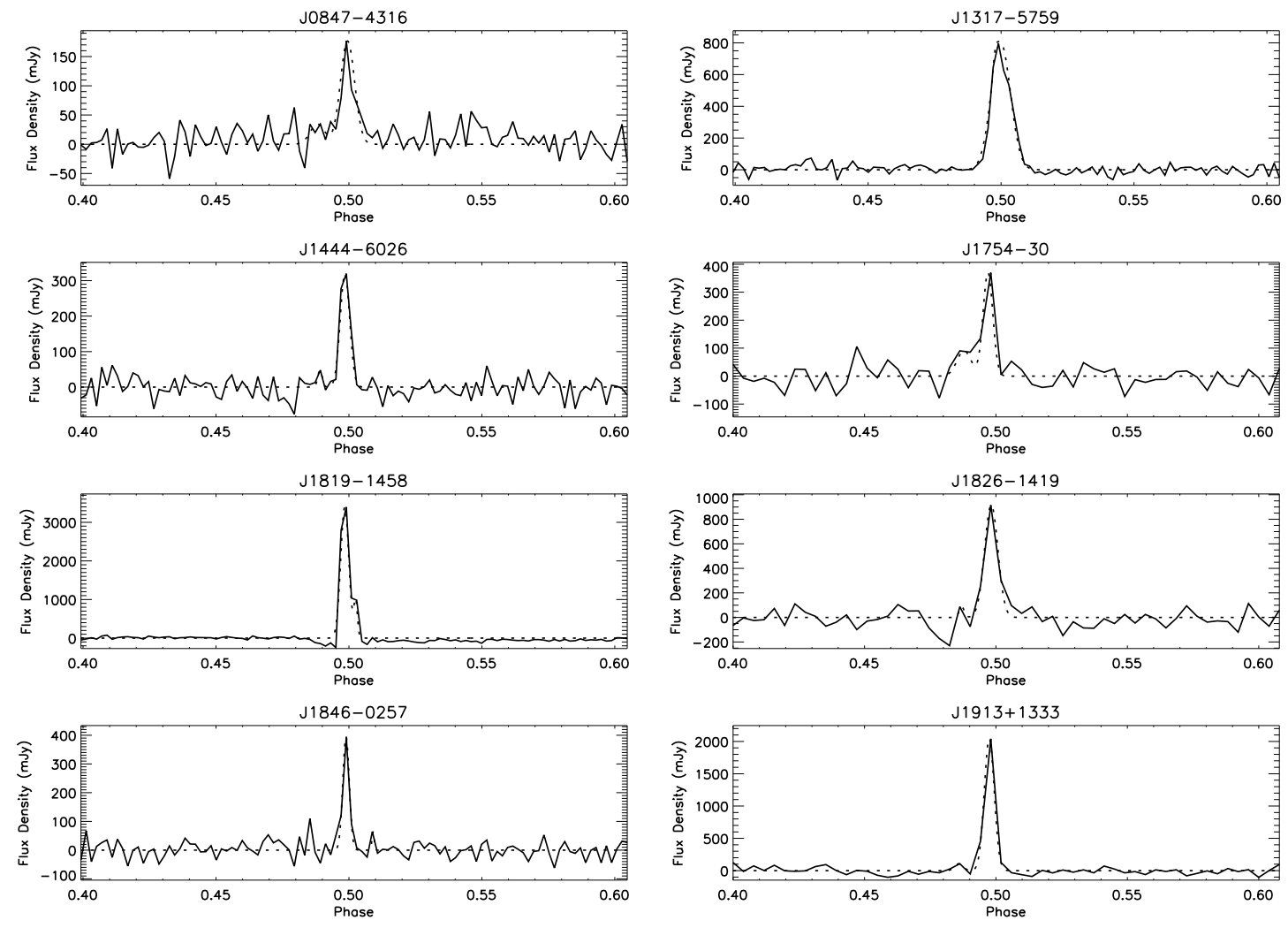

Figure 2.1: Pulse profiles of the brightest pulses detected in 1.4-GHz Parkes observations for the eight RRATs discussed in this paper. There are 512 bins across the pulse profile for each pulse. The best fit one-component (PSRs J1317-5759, J1444-6026, J1826-1419, J1846-0257, and J1913+1333) or two-component (PSRs J0847-4316, J1754-3014, and J1819-1458) Gaussian profile is overlaid as the dotted line. The flux density scale is different for each plot. 
less than the mean flux density uncertainties. The mean flux density, or the flux density averaged over one period, $P$, is therefore

$$
\langle S\rangle=\frac{1}{P} \sum_{i=1}^{512}\left(G_{1, \mathrm{i}}+G_{2, \mathrm{i}}\right) t_{\mathrm{samp}}
$$

where the product of $G_{1, \mathrm{i}}\left(+G_{2, \mathrm{i}}\right)$ and $t_{\text {samp }}$ represents the pulse energy (in $\mathrm{Jy} \cdot \mathrm{s}$ ) of the $i^{\text {th }}$ bin of the first (and second) fitted Gaussian profile. Equation 2.4 is a summation over all 512 bins of the period, not just the on-pulse region, so that the error propagation in calculating the uncertainty of $\langle S\rangle$ is not directly reliant on the size of the on-pulse region selected. From our $6 \sigma_{\mathrm{RN}}$ detection criterion, the mean flux density of the minimum detectable pulse is therefore

$$
\langle S\rangle_{\min }=6 \sigma_{\mathrm{RN}} \sqrt{\frac{t_{\mathrm{samp}}}{w}} \cdot \frac{w}{P}
$$

where $w$ is the width of a single pulse and we have defined the mean flux density $\langle S\rangle$ to be the flux density averaged over one period $P$. Values of these parameters are given in Table 2.2 .

The mean flux densities and standard deviations for all of the fitted Gaussian profiles for all the pulses at a single frequency are shown in Table 2.3 as $\langle S\rangle$ and $\sigma_{S}$, respectively. The numbers in parentheses following each value are the errors derived from including both statistical errors in the data and the $10 \%$ systematic errors for both $T_{\text {sys }}$ and $G$. 


\subsubsection{Pulse Distributions}

Once the flux density of each pulse was calculated for a given RRAT at a given frequency, we derived the complementary cumulative distribution of the flux densities,

$$
\operatorname{CCDF}(S)=1-\operatorname{CDF}(S)=1-\int_{0}^{S} \operatorname{PDF}\left(S^{\prime}\right) d S^{\prime}
$$

where $\operatorname{CDF}(S)$ is the cumulative distribution function (here normalized to one) which describes the probability of a pulse having a flux density less than a given $S$-value. We chose to compare the CCDFs of the RRATs because the CCDFs emphasize the largest flux density values of the distribution. This allows us to fit the entire distribution based on the strongest pulses. The CCDFs of pulse flux densities for various RRATS are shown in Figure 2.2. The left-hand cutoff of each distribution is $\langle S\rangle_{\min }$, given in Equation 2.5 and listed in Table 2.2. For some RRATs, like PSR J1819-1458, the flattening of the CCDF at low flux density values suggests that we are seeing the tail of the distribution and for other RRATs, like PSR J1754-3014, the lack of flattening near $\langle S\rangle_{\min }$ suggests that the pulses are the extreme end of a more normal flux density distribution. For these two RRATs, GBT observations with greater sensitivity support this, i.e. PSR J1819-1458 is still sporadic and PSR J1754-3014 looks much like a normal pulsar.

The distributions closely resemble the CCDF of the PDF given in Equation 2.1,

$$
\operatorname{CCDF}(S)=\frac{1}{2} \operatorname{erfc}\left(\frac{\ln S-\mu_{\ln }}{\sqrt{2} \sigma_{\ln }}\right)
$$



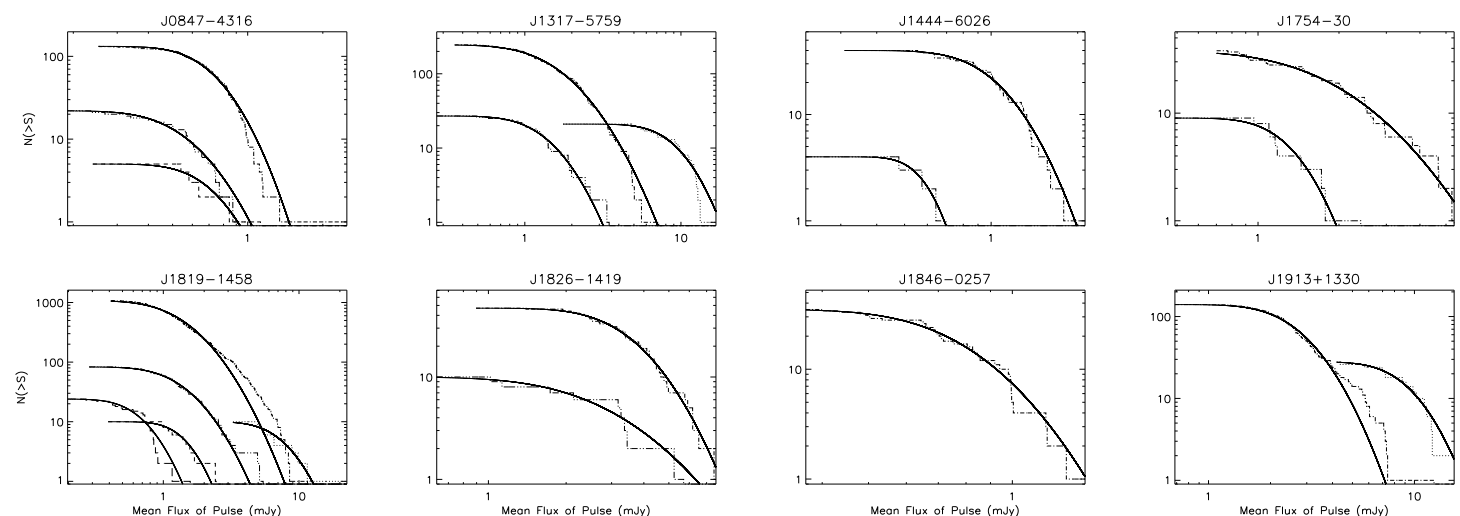

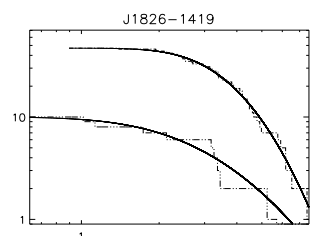

Mean Flux of Pulse (muy)

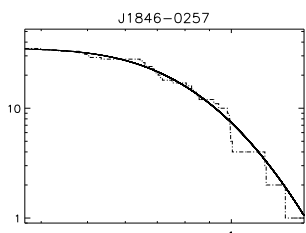

Mean Flux of Pulse (myy)

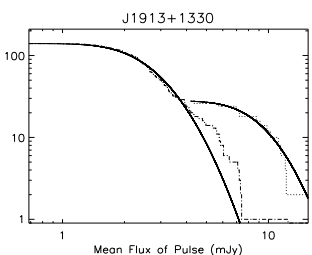

Figure 2.2: Complementary cumulative flux density distributions of RRATs. The dot, dash, dash-dot, dash-dot-dot-dot, and long-dash lines represent distributions from observations with central frequencies at 685, 1373, 1390, 1517, and $3029 \mathrm{MHz}$, respectively. The solid lines represent the best-fit log-normal CCDFs. The left hand cut-off for each distribution is $\langle S\rangle_{\min }$, listed in Table 2.2.

At the highest flux density values the log-normal distribution can be approximated by a power-law distribution. However, it can be clearly seen that in every case a power-law distribution (whose CCDF would appear as a straight-line fit to the data in Figure 2.2) does not fit the data as well as a log-normal distribution does. A log-normal distribution function was fit to each RRAT's flux density distribution at each observation frequency using a non-linear least-squares fitting algorithm. The quality of the log-normal distribution fit is quantified by performing the KolmogorovSmirnov (KS) test (see, e.g., Press et al., 1986) on the measured and log-normal distributions. The KS goodness-to-fit statistic is the maximum deviation between the cumulative flux density distribution and the CDF corresponding to Equation 2.1. Table 2.3 lists the significance level, $Q_{\mathrm{KS}}$, of the KS goodness-to-fit statistic; small values of $Q_{\mathrm{KS}}$ show that the cumulative flux density distribution is significantly different from a log-normal CDF. In most cases $Q_{\mathrm{KS}}>0.8$. The fitted distributions are shown as the dashed lines in Figure 2.2. 
The mean flux density of a RRAT at each frequency may be extracted from $\mu_{\ln }$ and $\sigma_{\ln }$ in Equation 2.7 as follows:

$$
\langle S\rangle_{\ln }=e^{\mu_{\ln }+\frac{1}{2} \sigma_{\ln }^{2}}
$$

Likewise, the standard deviation of the flux densities at the given frequency may be calculated:

$$
\sigma_{S \ln }=e^{\mu_{\ln }+\frac{1}{2} \sigma_{\ln }^{2}} \sqrt{e^{\sigma_{\ln }^{2}}-1}
$$

$\langle S\rangle_{\ln }$ and $\sigma_{S \ln }$ are both given in Table 2.3. The numbers in parentheses following each value are the errors derived from fitting the log-normal distribution function to the data.

Above flux densities of $\sim 4 \mathrm{mJy}$, the $1390 \mathrm{MHz}$ flux density distributions of PSRs J1819-1458 and J1913+1330 exhibit power-law tails in addition to the log-normal distributions. The log-normal distribution fit for PSR J1819-1458 is particularly poor, with $Q_{\mathrm{KS}}=0.06$. While three other distributions have lower values of $Q_{\mathrm{KS}}$ than the $1390 \mathrm{MHz}$ fit for PSR J1913+1330, it is the only one that appears to have a power-law tail. After subtracting the log-normal contribution from the distributions of PSRs J1819-1458 and J1913+1330, we fit power-law CCDFs for $S \geq 4 \mathrm{mJy}$. For a power-law PDF, given by Equation 2.2, the corresponding CCDF is $\propto S^{-K+1}$. The fitted power-law tails for the $1390 \mathrm{MHz}$ observations of PSRs J1819-1458 and J1913+1330 have $K=3.07(1)$ and 3.97(5), respectively, where the numbers in parentheses following measured or calculated values represent 
the $1 \sigma$ errors here throughout the rest of the dissertation.

\subsubsection{Spectral Indices}

Once the mean flux density is found at every frequency for a given RRAT, we may obtain the spectral index of the mean flux densities if there are at least two frequencies at which the RRAT was detected. Four of the RRATs considered here were detected in observations at two or more frequencies and three at three or more frequencies. After taking the logarithm of mean flux densities and the corresponding observation frequencies, we fit a straight line to the data. This analysis assumes that power-laws correctly describe the spectra.

Figure 2.3 shows mean flux densities derived from the best-fit log-normal distribution functions vs. frequency and the corresponding spectral indices. The error bar on each data point represents the scaled uncertainty in the mean flux density derived from fitting the log-normal CCDF, listed in Table 2.3. The solid line represents the best-fit power-law while the dotted lines represent the $1 \sigma$ uncertainty of the fit arising from the $1 \sigma$ uncertainties of both fitting parameters, i.e. the slope and vertical offset of the line.

For comparison, the spectral indices calculated from both $\langle S\rangle$ and $\sigma_{S}$ as well as $\langle S\rangle_{\ln }$ and $\sigma_{S \ln }$ are given for the seven RRATs that have multiple frequency detections as $\alpha_{\mathrm{SI}}$ and $\alpha_{\mathrm{SI} \text { ln }}$ respectively in Table 2.3. Since there are two fitting parameters for the power-law function, the reduced $\chi^{2}$ statistic is only calculated for the three RRATs that have pulses detected at more than two frequencies (i.e. 

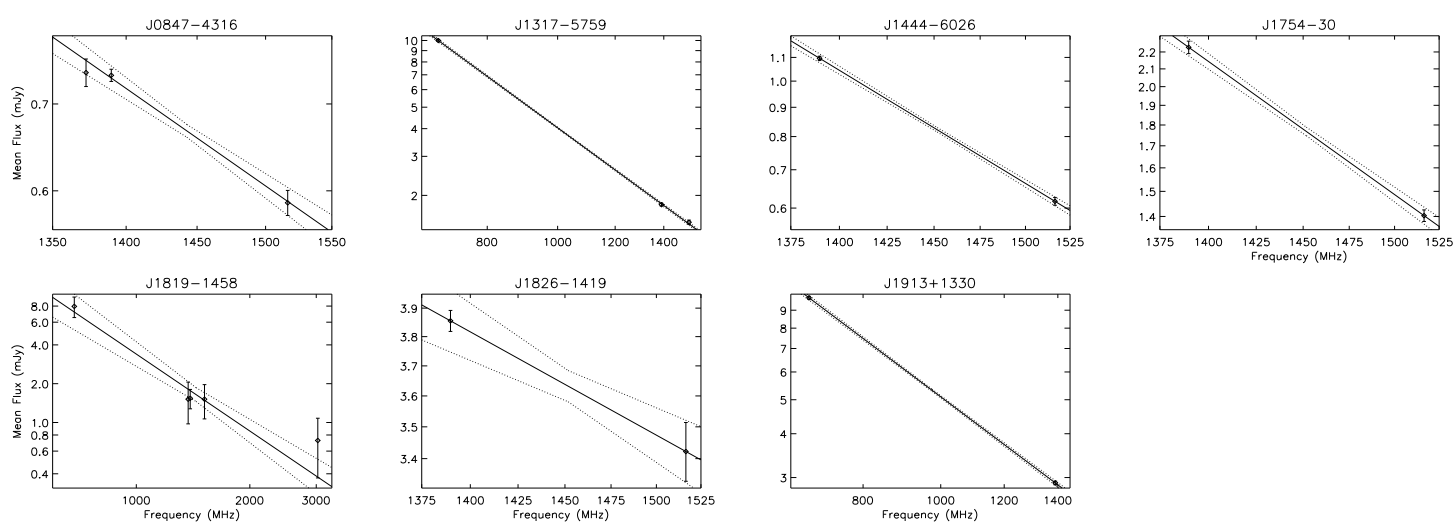

Figure 2.3: Spectral indices of RRATs derived from mean flux densities of the best-fit log-normal CCDFs at each frequency. Error bars on each flux density measurement are from the fitted log-normal distributions shown in Figure 2.2 and for cases where $\chi^{2}$ can be calculated (PSRs J0847-4316, J1317-5759, and J1819-1458) the errors are scaled such that $\chi^{2}=1$ (see text). Solid lines represent the best-fit spectral index. Dotted lines represent the $1 \sigma$ boundaries of the linear fit, both in slope and offset.

PSRs J0847-4316, J1317-5759, and J1819-1458). Assuming the noise of the RRATs' mean flux densities is white and well behaved, if $\chi^{2} \neq 1$ then the errors are either underestimated $\left(\chi^{2}>1\right)$ or overestimated $\left(\chi^{2}<1\right)$. In the cases where the reduced $\chi^{2}$ statistic could be calculated, mean flux density errors were collectively scaled by a factor $\kappa$ so that $\chi^{2}=1$. In doing this, we assume that the log-normal distribution is a perfect fit to the data. Scaling factors for the $1 \sigma$ errors of $\langle S\rangle$ and $\langle S\rangle_{\text {ln }}$ are given in Table 2.3 as $\kappa_{\mathrm{SI}}$ and $\kappa_{\mathrm{SI} \text { ln }}$, respectively.

All the RRATs with measured values of spectral indices seem to be welldescribed by a power-law with a negative spectral index. The mean spectral index based on the calculated mean flux densities is $\left\langle\alpha_{\mathrm{SI}}\right\rangle=-3.2(7)$, while the mean spectral index based on the mean flux densities derived from fitting log-normal distributions is $\left\langle\alpha_{\text {SI ln }}\right\rangle=-3.1(1)$. These mean values are significantly steeper than the mean spectral index for normal pulsars, $\left\langle\alpha_{\mathrm{SI}}\right\rangle=-1.8(2)$ (Maron et al., 2000). How- 
ever, only two of the seven RRATs (PSRs J1444-6026 and J1754-3014) fall outside of the range of normal pulsars. For these two RRATs, there are only four and nine detected pulses at $1517 \mathrm{MHz}$, respectively, one of only two observation frequencies at which they were detected, making it difficult get a good determination of the mean flux density at these frequencies. If only the RRATs with a wide observed frequency range $(>150 \mathrm{MHz})$ are considered (i.e. PSRs J1317-5759, J1819-1458, and $\mathrm{J} 1913+1330)$, then $\left\langle\alpha_{\mathrm{SI}}\right\rangle$ and $\left\langle\alpha_{\mathrm{SI} \ln }\right\rangle$ become $-1.7(1)$ and $-2.0(1)$, respectively. These values agree with the mean spectral index of normal pulsars within uncertainties. Further studies should determine whether the spectral indices of RRATs are the same as regular pulsars' spectral indices or if they are indeed steeper.

\subsubsection{Simultaneous Dual-Frequency Radio Observations of PSR J1819-1458}

Of the 527 epochs used for this analysis, 15 were dual-frequency observations using the 10-50 cm receiver, i.e. at 685 and $3029 \mathrm{MHz}$. While all 15 of these observations detected pulses of PSRs J1317-5759, J1819-1458, and J1913+1330 at $685 \mathrm{MHz}$, only PSR J1819-1458 was detected at $3029 \mathrm{MHz}$. This is consistent with the spectral indices and minimum detectable flux densities for each of the three RRATs. The mean flux densities for PSRs J1317-5759, J1819-1458, and $\mathrm{J} 1913+1330$ at $685 \mathrm{MHz}(\langle S\rangle=9.6(8), 9(2)$, and $9.4(9) \mathrm{mJy})$ when scaled to $3029 \mathrm{MHz}$ using the spectral indices $\left(\alpha_{\mathrm{SI}}=-2.35(3),-1.3(3)\right.$, and $\left.-1.6(1)\right)$ are $0.29(3), 1.3(6)$, and $0.9(2)$ mJy, respectively, cf. the minimum detectable mean flux density of a single pulse at $3029 \mathrm{MHz}$ of $0.24,0.20$, and $0.41 \mathrm{mJy}$. Only the scaled 
flux densities of PSR J1819-1458 are significantly (more than 5 times) greater than the minimum detectable mean flux density of a single pulse at $3029 \mathrm{MHz}$. Here we have used $\alpha_{\mathrm{SI}}$, not $\alpha_{\mathrm{SI} \text { ln }}$, because of the power-law tail of the $1390 \mathrm{MHz}$ flux density distribution of PSR J1819-1458 mentioned in Section 2.3.2; see Section 2.4 for more details.

Over four different epochs, PSR J1819-1458 was observed for 2.5 hours with this receiver, which equates to $\sim 2110$ rotations. During this time, ten pulses $(0.5 \%$ of the total rotations of the neutron star) were detected at $685 \mathrm{MHz}$ and 24 pulses (1.1\% of the total rotations of the neutron star) were detected at $3029 \mathrm{MHz}$, with only one of the pulses detected at both frequencies simultaneously. Therefore, if the detection at each frequency is random and independent from the other frequency, there is a $(10 / 2110) \times(24 / 2110)=0.0054 \%$ chance of seeing the pulse at both frequencies during the same rotation. The probability of not detecting the pulse at both frequencies is then $1-0.000054=99.9946 \%$ for any single rotation, and $(1-0.000054)^{2110}=89.3 \%$ for all 2110 rotations. The probability of detecting at least one coincident pulse from the two pulse trains if they are both random and independent of each other is then $10.7 \%$ and it is therefore possible that this one coincident pulse is due to chance. The spectral index calculated from PSR J1819-1458's one coincidentally detected pulse is $-2.0(7)$, which agrees with both $\alpha_{\mathrm{SI}}=-1.3(3)$ and $\alpha_{\mathrm{SI} \ln }=-2.0(3)$ within the $1 \sigma$ errors. 


\subsection{Discussion}

We have presented a new method for calculating spectral indices through using values from fitted flux density distributions. For sparse data sets with a small number of pulses, this method is more reliable than the direct method. This analysis highlights the similarities and differences between RRATs and other neutron stars. In most cases, the pulse amplitude distributions of RRATs exhibit log-normal behavior with evidence for power-law tails at the high flux density end of the distributions in at least two cases. RRATs' spectral behavior appears to follow power-law like behavior, similar to normal pulsars. Also, the coincident pulse rate of detected pulses in multifrequency observations is lower than expected when compared to the simultaneous pulse detection rates of other neutron stars (see below). Here, we discuss our results in the context of other studies.

As can be seen in Figure 2.2, most of the RRATs appear to have pulse amplitude distributions well described with log-normal PDFs (Equation 2.1), like normal pulsars. The two exceptions are the $1390 \mathrm{MHz}$ distributions of PSRs J1819-1458 and J1913+1330 observed at $1390 \mathrm{MHz}$. Note there may also be power-law tails for distributions of PSRs J1819-1458 and J1913+1330 at other frequencies, as well as for the other RRATs, that we may not have the sensitivity to detect - the $1390 \mathrm{MHz}$ flux density distributions of PSRs J1819-1458 and J1913+1330 make up two of the three distributions with the most detected pulses. These findings are similar to those reported by Kramer et al. (2003), who also observed a power-law tail for the flux density distributions of PSR B1133+16 which emerged from a log-normal 
distribution at higher frequencies $(\sim \mathrm{MHz})$ and was attributed to giant pulses. Similarly, the flux density distributions presented by Kramer et al. were also limited by their sensitivity and hence limited their ability to recognize the power-law tail of the distribution. The RRATs' power-law component can also be compared to XTE J1810-197, which exhibits both log-normal and power-law behavior (Serylak et al., 2009). Note that the power-law tails of the flux density distributions may be skewing the values of $\langle S\rangle_{\ln }$ (which assumes a log-normal distribution with no power-law tail) and hence affecting the value of $\alpha_{\mathrm{SIln}}$.

While the range of the RRATs' spectral indices is large $\left(-7 \leq \alpha_{\mathrm{SI}} \leq-1.3\right)$, and generally steeper than the mean spectral index for normal pulsars, the spectral indices of the three RRATs with the largest observed frequency range $(>150 \mathrm{MHz})$ are similar to those of normal pulsars. The spectral indices listed here exclude flat radio spectra, which have been observed for the two radio emitting magnetars (Camilo et al., 2006, 2007; Lazaridis et al., 2008), for all seven RRATs. The steeper nature of RRATs' spectral indices suggests a similarity with giant-pulsing pulsars. The giant pulses of $1937+21$ have a slightly steeper spectrum than the normal pulses, $\left\langle\alpha_{\text {SI GP }}\right\rangle=-1.8$ (Kinkhabwala \& Thorsett, 2000). The giant-pulsing millisecond PSR J1824-2452A has individual pulses with spectra as steep as -5.4 , but the average giant pulse spectral index cannot be steeper than -4.4 to be consistent with high-frequency detections (Knight et al., 2006). The Crab pulsar giant pulses have a mean spectral index of $\left\langle\alpha_{\mathrm{SIGP}}\right\rangle=-3.4(4)$, which is comparable to the spectral index of its normal pulses, -3.0 (Sallmen et al., 1999).

In order to understand the robustness of our spectral index measurements, we 
ran Monte Carlo simulations to ensure there was no bias in our fitting algorithm. We fit a spectral index to random flux densities with a mean of $\langle S\rangle$ and a standard deviation given by the $1 \sigma$ errors of $\langle S\rangle$ listed in Table 2.3 at all frequencies. The results of our simulations were consistent with the values of $\alpha_{\mathrm{SI}}$ and $\alpha_{\mathrm{SI} \text { ln }}$ to well within their $1 \sigma$ errors and showed no evidence of a fitting bias that might make the spectral indices more negative.

The dual-frequency radio observations of PSR J1819-1458 suggest a somewhat narrowband $(<2 \mathrm{GHz})$ emission mechanism, with only one coincident pulse out of the 33 pulses detected at both 685 and $3029 \mathrm{MHz}$, which yields a simultaneous detection rate of roughly $3 \%$ ( $0.05 \%$ of the total rotational periods observed, $\left.0.4 \mathrm{hr}^{-1}\right)$. Giant pulses also appear to be narrowband when observed simultaneously over bandwidths $>1 \mathrm{GHz}$. The Crab pulsar has a $70 \%$ simultaneous detection rate of giant pulses observed at 0.6 and $1.4 \mathrm{GHz}(0.8 \mathrm{GHz}$ bandwidth) Sallmen et al. (1999), a 6\% simultaneous detection rate at 584 and $2228 \mathrm{MHz}$ (1.6 GHz bandwidth) Kostyuk et al. (2003), and a 6\% simultaneous detection rate at 600 and $4850 \mathrm{MHz}$ (4.25 GHz bandwidth) Popov et al. (2008). Popov et al. (2008) also noted that not all the pulses detected at both 600 and $4850 \mathrm{MHz}$ were detected at the intermediate observation frequency of $1650 \mathrm{MHz}$.

Overall, the percentage of PSR J1819-1458's coincident pulses is less than the percentage of coincident pulses found in other multiple-frequency studies but is most similar to the results for the Crab pulsar (see above). While the dualfrequency observations provide an upper limit on the emission mechanism bandwidth of PSR J1819-1458 of 2 GHz, the observation bandwidth of single obser- 
vations can offer a lower limit. We do not see any evidence of narrowband behavior throughout the $5120.5-\mathrm{MHz}$ frequency channels for the brightest pulses detected from PSR J1819-1458 at $1390 \mathrm{MHz}$. Therefore, the narrowband nature of the emission mechanism bandwidth seems to lie somewhere between 0.25 and $2 \mathrm{GHz}$.

Thus far, this is the first reporting of radio spectral bulk statistics for RRATs. This analysis will benefit as more observations are performed and new RRATs are discovered. The ideal situation would be to observe these RRATs with simultaneous multiple radio frequency observations so that multi-frequency analysis may be performed on individual pulses. Joint observations at radio and other wavelengths such as infrared and X-ray may also offer further insight to understanding the emission mechanisms of these peculiar neutron stars. 


\section{Chapter 3}

\section{Simultaneous X-ray and Radio Observations of Rotating Radio}

\section{Transient J1819-1458}

This chapter was originally published as a refereed paper in the Astrophysical Journal (ApJ) by J. J. Miller, M. A. McLaughlin, N. Rea, K. Lazaridis, E. F. Keane, M. Kramer, and A. Lyne in October of 2013. Minor wording changes from the original accepted paper have been made where appropriate.

\subsection{Introduction}

There are over 2000 known pulsars, ${ }^{1}$ with roughly 70 of these labeled as RRATs $^{2}$; see Keane \& McLaughlin (2011) for a recent review. The single pulses of RRATs have similar widths and intensities to single pulses of other pulsars, but despite an underlying periodicity at the neutron star's rotational period, radio pulses are sporadically detected. It is unclear why the emission of these objects is so sporadic, and numerous theories have been put forward which rely on both internal factors, such as RRATs may be dying or extreme nulling pulsars (Zhang et al., 2007), or external factors such as modulation of the emitted pulses from a radiation belt similar to planetary magnetospheres (Luo \& Melrose, 2007) or disturbances from the pulsar's asteroid belt (Cordes \& Shannon, 2008).

\footnotetext{
${ }^{1}$ http://www.atnf.csiro.au/people/pulsar/psrcat/

${ }^{2}$ http://www.as.wvu.edu/ pulsar/rratalog
} 
PSR J1819-1458 has a spin period of $P=4.26 \mathrm{~s}$, with a pulse detected roughly every three minutes in Parkes observations above a flux density of $S=0.6 \mathrm{mJy}$ at $1.4 \mathrm{GHz}$ (McLaughlin et al., 2006). It has a characteristic age of $\tau_{c}=117 \mathrm{kyr}$, a spin-down luminosity of $\dot{E}_{\text {rot }}=3 \times 10^{25} \mathrm{~W}$, and a high inferred surface magnetic field strength of $B=5 \times 10^{9} \mathrm{~T}$ at the magnetic equator. The distance estimate from its DM of $196.0 \pm 0.4 \mathrm{pc} \mathrm{cm}^{-3}$ (Esamdin et al., 2008) and the Galactic electron density model of Cordes \& Lazio (2002) is $3.6 \mathrm{kpc}$ with considerable (at least 25\%) uncertainty.

A previous 43 ks observation of PSR J1819-1458 by XMM-Newton (McLaughlin et al., 2007) found best-fit spectral models with neutral hydrogen column densities $n_{\mathrm{H}} \sim 7 \times 10^{21} \mathrm{~cm}^{-2}$, temperatures near $k T \sim 0.14 \mathrm{keV}$, a single absorption line near $\sim 1 \mathrm{keV}$, and unabsorbed fluxes $\sim 2 \times 10^{-12} \mathrm{erg} \mathrm{s}^{-1} \mathrm{~cm}^{-2}(0.3-5 \mathrm{keV})$, which yield a blackbody emission radius (at infinity assuming a $3.6 \mathrm{kpc}$ distance) of $R=8_{-3}^{+7} \mathrm{~km}$. This temperature is expected for a $10^{4}-10^{5}$ year-old cooling neutron star's emission (Yakovlev \& Pethick, 2004), generally in agreement with PSR J1819-1458's age. The unabsorbed fluxes yield luminosities which exceed the spin-down luminosity of PSR J1819-1458 by a factor of $L_{0.3-5.0 \mathrm{keV}} / \dot{E}_{\mathrm{rot}} \simeq$ $4 \times 10^{33} / 3 \times 10^{32} \simeq 6-18$, depending on the spectral model, which is possible given the thermal origin of the X-ray emission. The results reported by McLaughlin et al. (2007) are consistent with both a chance Chandra observation of PSR J1819-1458 (Reynolds et al., 2006) as well as deeper Chandra observations of PSR J1819-1458 (Rea et al., 2009; Camero-Arranz et al., 2013). The absorption line seen with XMMNewton by McLaughlin et al. (2007) was confirmed with Chandra by Rea et al. 
(2009), which rules out instrumentation as the cause. The latter Chandra observations also revealed a bright pulsar wind nebula around PSR J1819-1458, with an inferred X-ray efficiency of $\eta_{X}=L_{\text {pwn:0.5-8.0keV }} / \dot{E}_{\text {rot }}=6.0 \times 10^{31} / 3 \times 10^{32} \simeq 0.2$.

Several spectral models can be used to explain the absorption in the spectrum of PSR J1819-1458. Possible explanations are elements in the ISM, elements in the neutron star's atmosphere, or cyclotron absorption. A cyclotron proton resonant scattering model yields the magnetic field strength $B_{\mathrm{cy}}=1.6 E_{\mathrm{cy}}(\mathrm{keV}) / y_{\mathrm{G}} 10^{10} \mathrm{~T}$, where $E_{\text {cy }}$ is the cyclotron proton energy, and $y_{\mathrm{G}}=\left(1-2 G M / c^{2} R\right)^{1 / 2}$ is the gravitational redshift factor $\left(\sim 0.77\right.$ using a canonical neutron star mass $M=1.4 M_{\odot}$, and a canonical neutron star radius $R=10 \mathrm{~km}$ ). If the cyclotron resonance was due to electrons and not protons, the inferred magnetic field strength would be $m_{p} / m_{e}=1.8 \times 10^{3}$ times weaker, making proton cyclotron resonance more likely due to the high inferred surface magnetic field strength of PSR J1819-1458.

Absorption lines have been observed in several other isolated neutron stars. These include some X-ray Isolated Neutron Stars (Hohle et al., 2012; see Turolla, 2009 and Kaplan \& van Kerkwijk, 2011 for recent reviews), which have absorption lines reported at lower energies $(300-700 \mathrm{eV})$ than those observed for PSR J1819-1458. Furthermore, another rotation-driven pulsar, PSR J1740+1000, has been shown to have an absorption feature around $600 \mathrm{eV}$ (Kargaltsev et al., 2012). It is unclear why some neutron stars exhibit absorption and others do not, with various explanations offered for these absorption lines, e.g. proton cyclotron resonances and atomic transitions in light elements (Turolla, 2009; Kaplan \& van Kerkwijk, 2011). 
While spectral observations are important for probing the pulsar environment, X-ray timing observations can also be useful to learn about emission mechanisms, especially when combined with synchronous radio observations. If radio pulses are correlated with X-ray photons, then a combined mechanism could be responsible for radio and high-energy emission. Such tests have been done to correlate radio giant pulses from the Crab pulsar pulses with non-thermal X-ray and gamma-ray photons. No correlation was found in these studies (Bilous et al., 2011) but a correlation was found between radio giant pulses and optical emission (Shearer et al., 2003), suggesting an overall increase in particle density could be responsible for the giant pulses. In the case of RRATs, Zhang et al. (2007) suggest that we should expect an increase in both non-thermal and thermal X-ray emission close to radio pulse detection times if their sporadicity is due to their reactivation model. This model suggests that the pulsar is only active when the conditions in its magnetosphere allow for pair production which instigates coherent radio emission that results in non-thermal X-ray photons and thermal emission from polar-cap heating. If the pulsar is always active and the sporadicity is due to radio emission direction reversal, however, then the non-thermal and polar-cap heating will always be present and we should therefore not see an increase in X-ray emission close to radio pulse detection times.

We were awarded $94 \mathrm{ks}$ of XMM-Newton time to improve the accuracy of the spectral parameters, determine the origin of the absorption lines, search for evidence of a non-thermal power-law structure in the spectrum, and explore whether the Xray and radio emission is correlated. We were also awarded time on the GBT, the 
Parkes radio telescope, and the Effelsberg radio telescope for simultaneous radio observations. We report here on the results of these observations. In Section 3.2 we describe the X-ray properties of PSR J1819-1458, quantifying absorption features and the possibility of a power-law tail. In Section 3.3 we describe the star's radio properties. In Section 3.4 we compare the observed profile at both wavelengths and present the correlation of pulse arrival times. Finally, we draw some conclusions in Section 3.5.

\subsection{X-Ray Observations and Analysis}

We observed PSR J1819-1458 with XMM-Newton for 94 ks on 2008 March 31. These data were taken with EPIC-PN in Full Frame mode and the two MOS with the central CCD in Small Window mode, as was done by McLaughlin et al. (2007). The time resolutions of the EPIC-PN in Full Frame mode and two MOS

CCDs in Small Window mode are $73.4 \mathrm{~ms}$ and $0.3 \mathrm{~s}$, respectively. PSR J1819-1458 appeared as a point source with the following J2000 coordinates: right ascension $\alpha=18^{\mathrm{h}} 19^{\mathrm{m}} 34^{\mathrm{s}}$ and declination $\delta=-14^{\circ} 58^{\prime} 04^{\prime \prime}\left(4^{\prime \prime}\right.$ error in each coordinate, where all the errors in this dissertation are stated at the $1 \sigma$ confidence level), consistent with previous X-ray observations and the position derived from radio timing. We could not distinguish the $\sim 5^{\prime \prime} .5$ extended emission region detected by Rea et al. (2009) with Chandra, which has a spatial resolution of $\sim 0^{\prime \prime} .5$ (Weisskopf et al., 2002; Garmire et al., 2003) compared to the $\sim 6^{\prime \prime}$ spatial resolution of XMM-Newton (Watson et al., 2009). 
The timing and spectral analyses were done using the XMM-Newton Scientific Analysis System (SAS) tools, ${ }^{3}$ version 12.7.0. The Current Calibration File (CCF) was built using the cifbuild command on the SAS tools Web site, ${ }^{4}$ using the observation date 2008-03-31T14:06:38. In order to exclude events not associated with the pulsar, e.g. solar flares, we defined good time intervals (GTIs) by binning all of the PN and MOS detection times, as well as the detection times from within a $20^{\prime \prime}$ circular radius centered on the source position, into $10 \mathrm{~s}$ intervals. We then identified time intervals with excessive photon counts which were not confined to the source region, as determined by visual inspection (areas dominated by non-zero baselines and count rates greater than 100 counts per $10 \mathrm{~s}$ time intervals on the PN detector), and excluded those time intervals from the GTIs and our further analysis. Multiple GTI ranges were tested for both timing and spectral analysis. Our analysis resulted in three GTIs for each of the PN, MOS1, and MOS2 detectors. This excluded large bursts at the beginning and end of the observation which were not confined to the source regions; these GTIs span $68.6 \mathrm{ks}$ (19 hr) from MJD 54556.8 to MJD 54557.6 with each detector having three small interruptions, each spanning $3.5-15.6 \mathrm{~s}$, as shown in Table 3.1. The GTI and photon arrival times were barycentered to the center of the solar system using the XMM analysis tool barycen and the X-ray derived position.

\footnotetext{
${ }^{3} \mathrm{http}: / / \mathrm{xmm}$. esa.int/sas/

${ }^{4} \mathrm{http}: / /$ xmm.vilspa.esa.es/external/xmm_sw_cal/calib/cifbuild.shtml
} 
Table 3.1: X-ray Good Time Intervals

\begin{tabular}{lccc}
\hline \hline & PN & MOS1 & MOS2 \\
\hline GTI 1 MJD span & $54556.8164062-54556.8496346$ & $54556.8164062-54556.8494625$ & $54556.8164062-54556.8503720$ \\
GTI 2 MJD span & $54556.8496771-54556.8504032$ & $54556.8496430-54556.8503653$ & $54556.8505225-54557.6338842$ \\
GTI 3 MJD span & $54556.8505407-54557.6093750$ & $54556.8505158-54557.6093750$ & $54557.6339443-54557.6093750$ \\
\hline
\end{tabular}

Good Time Intervals (GTIs) for the PN, MOS1, and MOS2 detectors. See Section 3.2 for details.

\subsubsection{Timing Analysis}

Our time resolution is sufficient for studying the pulse profile because of the long period of the pulsar. For timing analysis we included all PN and MOS events within the GTIs satisfying a PATTERN $\leq 12$ requirement (i.e., allowing for single, double, triple, and quadruple events). To ensure extraction of at least $90 \%$ of the source photons, we chose a $20^{\prime \prime}$ circular radius centered on the source position in the data. We also extracted background counts from four nearby $20^{\prime \prime}$ circular region free of point sources and on the same central CCD as the source region to measure the average background rate. The photon arrival times were folded with the radio timing ephemeris of PSR J1819-1458 using TEMPO. ${ }^{5}$ The data were folded for a combination of 99 trial values of the number of pulse phase bins (2-100 bins), 1969 values of minimum energy $E_{\min }(0.155-9.995 \mathrm{keV})$, and 1969 values of maximum energy $E_{\max }(0.160-10 \mathrm{keV})$, creating $\sim 384$ million profiles. For each trial number of phase bins, value of $E_{\min }$, and value of $E_{\max }$, a $\chi^{2}$ value was calculated for a fit of the folded profile to a flat (i.e. random) distribution. The background rate subtracted X-ray profile with the lowest probability of being drawn from a flat

\footnotetext{
${ }^{5}$ http://www.atnf.csiro.au/research/pulsar/tempo/
} 
distribution, $P=10^{-52.1}$, has 10 phase bins, $E_{\min }=0.5 \mathrm{keV}$, and $E_{\max }=2.6 \mathrm{keV}$ and is shown in Figure 3.1. Of the 6630 total PN photons within the $20^{\prime \prime}$ radius, 5692 fall within this energy range.

A sinusoid model should provide a good fit for the X-ray profile arising from the rotating beam model explained in Chapter 1 , and so we fit the model to the Xray profile to determine the peak using a least-squares fitting routine. When adding a second-order sinusoid to the fit, $F(t)=A_{1} \cos \left(2 \pi\left(x-\phi_{0}\right)\right)+A_{2} \cos \left(4 \pi\left(x-\phi_{0}\right)\right)$, the reduced $\chi^{2}$ is decreased from 2.3 to 1.0. Similarly, fitting a Gaussian function produces a fit with reduced $\chi^{2}=1.0$. The phase of the peaks of both the double sinusoid and the Gaussian function was $0.02 \pm 0.01$, where phase zero is the peak of the radio pulse profile. These fits are also shown as the dotted and dashed line in Figure 3.1, respectively.

The X-ray pulse profile has a $0.5-2.6 \mathrm{keV}$ intrinsic pulsed fraction, defined as $\left(F_{\max }-F_{\min }\right) /\left(F_{\max }+F_{\min }\right)$, where $F_{\max }$ and $F_{\min }$ are the minimum and maximum background-corrected counts of the X-ray pulse profile, of $(33.9 \pm 0.9) \%$, using 10 bins and assuming Poisson (i.e. $\sqrt{N}$ ) errors. Previous background-corrected pulsed fractions reported for PSR J1819-1458 are $(34 \pm 6) \%,(28 \pm 7) \%$, and $(49 \pm 10) \%$ for the $0.3-5 \mathrm{keV}, 0.3-1 \mathrm{keV}$, and $1-5 \mathrm{keV}$ energy ranges, respectively (McLaughlin et al., 2007), and $(37 \pm 3) \%$ for the $0.3-5 \mathrm{keV}$ energy range (Rea et al., 2009). Pulsed fractions measured within the same energy ranges with our data set yields $(31.0 \pm 0.8) \%,(30 \pm 1) \%$, and $(49 \pm 1) \%$ for $0.3-5 \mathrm{keV}, 0.3-1 \mathrm{keV}$, and $1-5 \mathrm{keV}$, respectively. 


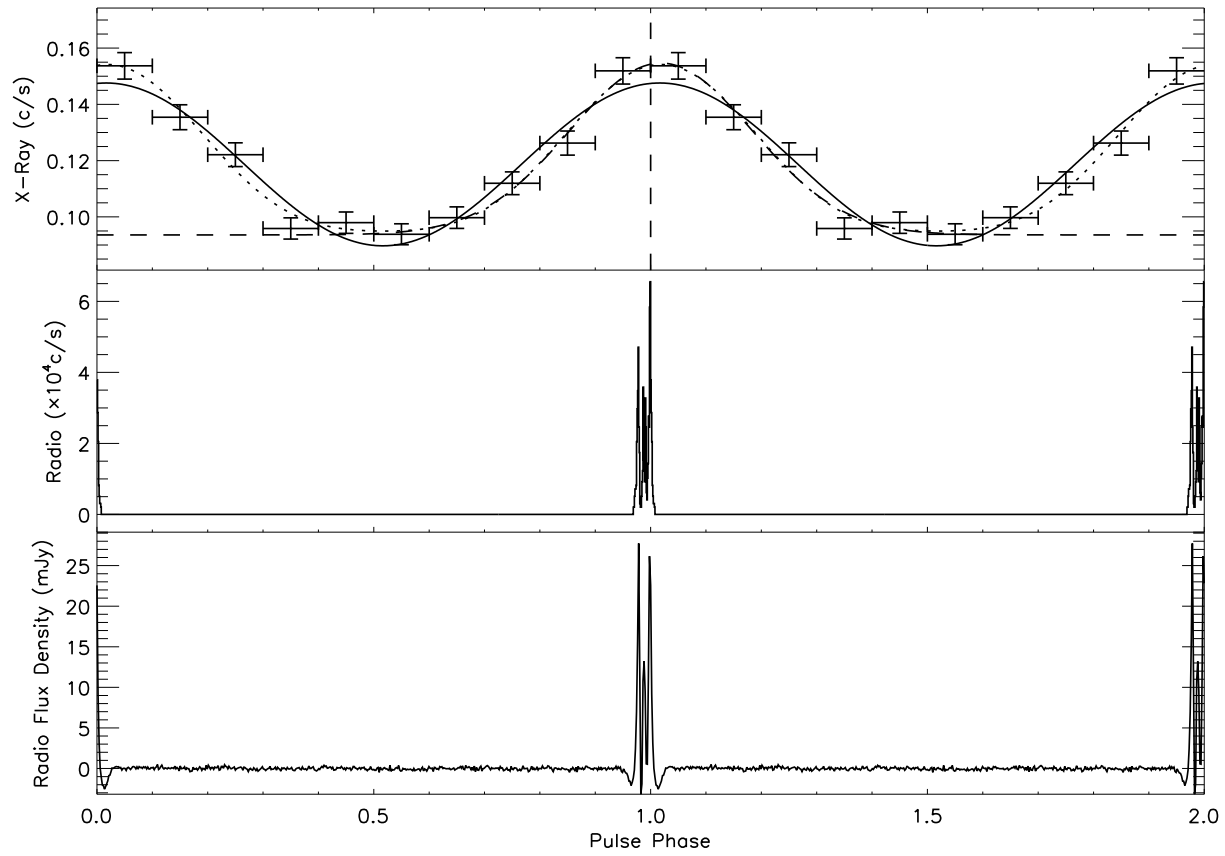

Figure 3.1: X-ray and radio profiles of PSR J1819-1458 folded using the radio ephemeris. Top: The background-corrected X-ray profile consists of 10 phase bins over one rotational period, which consists of XMM-Newton PN and MOS detected photons within the $0.5 \mathrm{keV}<E<2.6 \mathrm{keV}$ energy range within the GTIs and PATTERN $\leq 12$, summing up to $\sim 17 \mathrm{hr}$ of observation time. The horizontal and vertical bars indicate the size of the phase bins and the $\sqrt{N}$ errors. The solid, dotted, and dashed lines indicate the single sinusoid, two sinusoid, and Gaussian fits to the profile (fit over the $0.5-1.5$ phase range), respectively. Note that the dotted and dashed lines overlap considerably. The vertical dashed line indicates the peak of the radio pulse profile (phase $=1.0$ ). Middle: Radio pulse count histogram created by using the radio ephemeris to assign a phase to each barycentered pulse detected by the $7.7 \mathrm{hr}$ observation of the GBT at an observing frequency of $2 \mathrm{GHz}$, and then binning all the radio pulse arrival times into a 2048 bin histogram. Bottom: Radio flux density profile formed from pulses detected using the $7.7 \mathrm{hr}$ observation of the GBT at an observing frequency of $2 \mathrm{GHz}$. Flux densities were calculated by normalizing the scale of each detected pulse's off-pulse noise to the radiometer noise, then averaging all the pulses together. The dips preceding and following the pulse are due to digitization of the signal (e.g. Jenet \& Anderson, 1998). The profile is shown twice in all plots for clarity. 


\subsubsection{Spectral Analysis}

For spectral analysis, we selected photons from the PN detectors with a more stringent PATTERN $\leq 4$ requirement (i.e. allowing for single and double events), as the background will affect results more significantly. As in the timing analysis, we extracted the source photons from within a $20^{\prime \prime}$ circular radius centered on the source position, which yielded 6974 total events in a $0.5-2.0 \mathrm{keV}$ energy range. We also extracted background counts from four $20^{\prime \prime}$ circular regions centered on off-source positions free of point sources and on the same central CCD as the source region. The spectrum was then rebinned so that there were at least 30 counts per spectral bin so that we could use the $\chi^{2}$ statistic. $^{6}$ Additionally, we similarly processed the data from McLaughlin et al. (2007) and added the two observations together with the XSPEC command mathpha with a Gaussian error propagation method to create the spectrum shown in Figure 3.2. We also processed the MOS1 and MOS2 detections from the observation as well as from McLaughlin et al. (2007) also shown in Figure 3.2. We will only discuss PN spectral analysis hereafter, but both of our MOS spectra model fits are in agreement with the PN spectral analysis.

We restricted the energy range of our spectral fitting to $0.5-2.0 \mathrm{keV}$. This is narrower than that used for timing as at higher energies; the spectrum count rates were comparable to the background region count rates. We were unable to fit a spectral model with $\chi^{2}<2$ without addressing a feature in the residuals of the fits near $\sim 0.5 \mathrm{keV}$. McLaughlin et al. (2007) ignored the $0.5 \mathrm{keV}$ feature by excluding the $0.50-0.53 \mathrm{keV}$ energy range from their spectral fitting, but mentioned that an

\footnotetext{
${ }^{6}$ https://heasarc.gsfc.nasa.gov/xanadu/xspec/manual/manual.html
} 


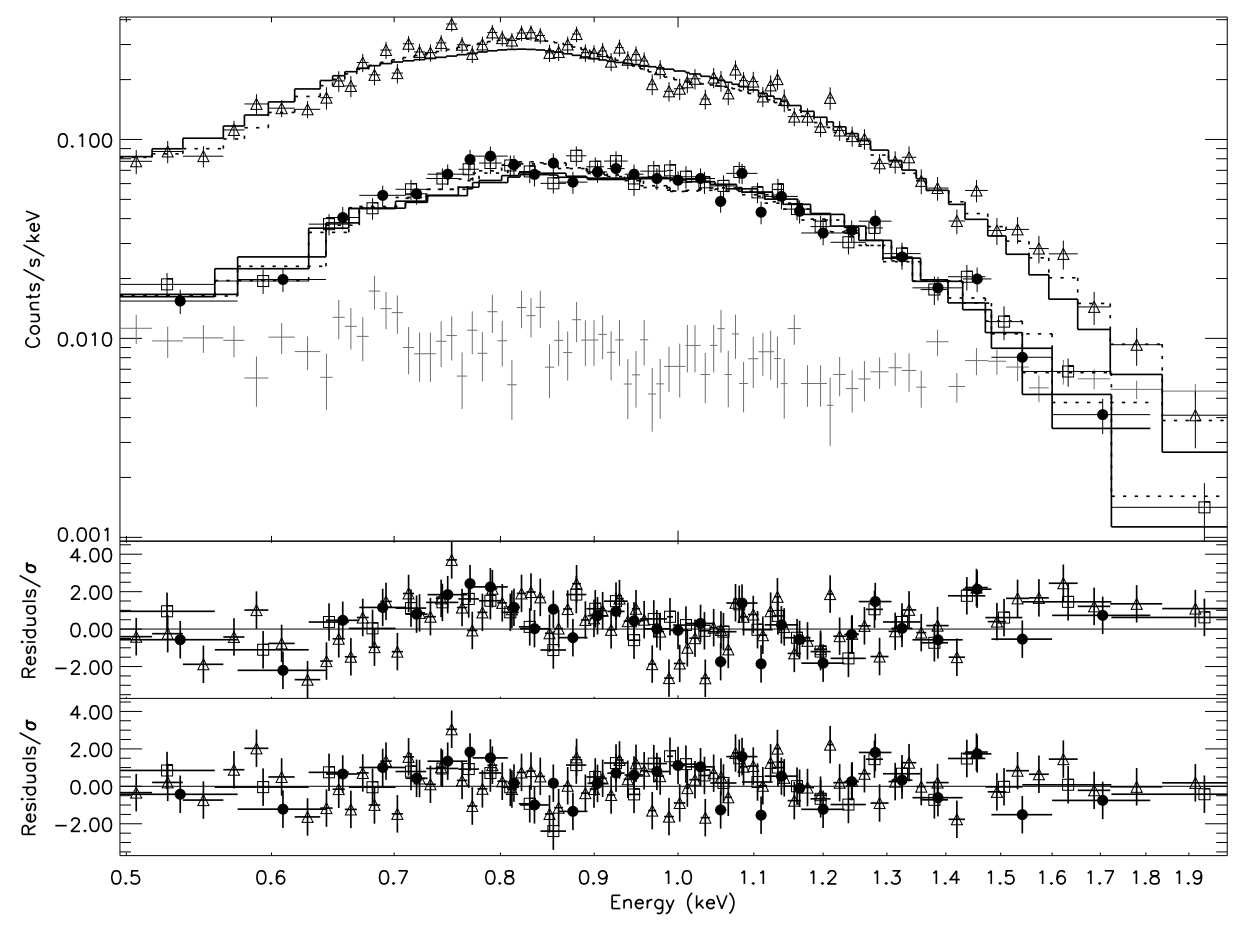

Figure 3.2: X-ray spectrum of PSR J1819-1458 using photons with energies in the 0.5-2.0 keV range and PATTERN $\leq 4$ from both our observation and McLaughlin et al. (2007). Data have been rebinned for plotting purposes by a factor of two from 157, 63, and 66 bins to 78, 30, and 33 bins for PN, MOS1, and MOS2, respectively. Top: The dark crosshairs indicate the PN (triangles), MOS1 (filled circles) and MOS2 (squares) source spectra, respectively. The light crosshairs represent the PN background spectrum. The solid lines indicate the simplest model fit, a blackbody with interstellar absorption, an underabundance of oxygen, and solar abundances from Lodders (2003) for elements other than hydrogen and oxygen (vphabs*bbody); while the dotted lines indicate one of the best model fits (vphabs*gabs*gabs*bbody), which also includes two Gaussian absorption lines around 1.0 and $1.3 \mathrm{keV}$. Middle: Normalized PN (triangles), MOS1 (filled circles) and MOS2 (squares) residuals for the vphabs*bbody model. Bottom: Normalized PN (triangles), MOS1 (filled circles) and MOS2 (squares) residuals for the vphabs*gabs*gabs*bbody model. 
underabundance of oxygen could explain it. We found that the oxygen edge in the XSPEC model vphabs fit our $\sim 0.5 \mathrm{keV}$ feature well and included it as well as the 0.50-0.53 keV energy range in all of our model fits. Solar abundances from Lodders (2003) were assumed for elements other than neon and oxygen. We also investigated fitting our spectral models to energy ranges above $2.0 \mathrm{keV}$, where it looked like a possible power-law tail may have been present, but attempts to fit the background dominated portion of the spectrum yielded unacceptable $\chi^{2}$ values.

Modeling the blackbody spectrum without fitting for the $1.0 \mathrm{keV}$ feature results in $\chi^{2} \sim 1.4$. Adding an absorption model around $1.0 \mathrm{keV}$, modeled as either an empirical Gaussian absorption or as cyclotron absorption, yields a better fit, $\chi^{2} \sim 1.2$ (see Table 3.2). Using an underabundance of neon to explain this feature as was done by McLaughlin et al. (2007) does not yield as good a fit as either the Gaussian or cyclotron absorption. While it is possible that the spectrum could also consist of two blackbody components, cooler emission from the surface along with a smaller hotspot, fitting yielded $\chi^{2}=1.19$ but with large blackbody emission radii errors, (see Table 3.2). Furthermore, the residuals suggest a second feature around $1.3 \mathrm{keV}$, so we tried to add another Gaussian absorption line to the model, resulting in $\chi^{2}=1.09$. We ran Monte Carlo simulations to assess the significance of the addition of the second absorption feature (see Rea et al. (2005) for further details), and found a significance of $\sim 3 \sigma$ for its addition to the continuum plus one feature model, i.e. $>99 \%$ likelihood of two absorption lines rather than just one. The two-line model is then preferred at a $3 \sigma$ significance, with $\chi^{2}=1.09$ (see Table 3.2). We did not include the two cyclotron absorption model even though it fit equally 
well as the others because the two energies are not harmonically related. We also tested the XSPEC neutron star atmosphere model nsa which yielded parameters in agreement with those found in Table 3.2. We performed a phase-resolved analysis, dividing the observation in the on-pulse spectra $(0.0-0.25$ and $0.75-1.0$ pulse phase in Figure 3.1) and off-pulse spectra (0.25-0.75 pulse phase in Figure 3.1). Results of the spectral fits to the on- and off-pulse spectra agreed with the parameters fit to the phase-integrated spectra within the parameter uncertainties.

The Leiden/Argentine/Bonn Survey of Galactic H i map (Kalberla et al., 2005) and Dickey and Lockman H $\mathrm{I}$ in the Galaxy map (Dickey \& Lockman, 1990) quote the total hydrogen column density along the line of sight of PSR J1819-1458 as $1.25 \times 10^{22} \mathrm{~cm}^{-2}$ and $1.64 \times 10^{22} \mathrm{~cm}^{-2}$, respectively, using a weighted average of all points within one degree of PSR J1819-1458. Since the maps represent column densities along the entire line of sight including hydrogen beyond the pulsar, it is reassuring that the hydrogen column densities in Table 3.2 are generally less than the map measurements. He et al. (2013) found an empirical relationship between $n_{\mathrm{H}}$ and DM for radio pulsars of $n_{\mathrm{H}} /\left(10^{20} \mathrm{~cm}^{-2}\right)=0.30_{-0.09}^{+0.13} \times\left(\mathrm{DM} / \mathrm{pc} \mathrm{cm}^{-3}\right)$, which implies an average radio pulsar ionization rate of $10_{-3}^{+4} \%$. When applied to PSR J1819-1458, this relation implies $n_{\mathrm{H}}=0.30_{-0.09}^{+0.13} \times\left(196.0 \pm 0.4 \mathrm{pc} \mathrm{cm}^{-3}\right)=0.6_{-0.2}^{+0.3} \times 10^{22} \mathrm{~cm}^{-2}$, which agrees with three of the six fitted models shown in Table 3.2. 
Table 3.2: Spectral Fits for PSR J1819-1458 with EPIC-PN

\begin{tabular}{lcccccc}
\hline \hline & Blackbody (BB) & BB $\times$ Neon & BB $\times$ Gaussian & BB $\times$ Cyclotron & BB + BB & BB $\times$ Gaussian $\times$ Gaussian \\
\hline$n_{\mathrm{H}}$ & $0.9 \pm 0.1$ & $0.88_{-0.08}^{+0.10}$ & $1.244 \pm 0.009$ & $1.174 \pm 0.009$ & $1.4_{-0.2}^{+0.3}$ & $0.88 \pm 0.01$ \\
$n_{O}$ & $0.3_{-0.3}^{+0.2}$ & $0.7_{-0.3}^{+0.2}$ & $0.82 \pm 0.02$ & $0.87 \pm 0.02$ & $0.8_{-0.2}^{+0.1}$ & $0.69 \pm 0.03$ \\
$n_{\mathrm{Ne}}$ & - & $3.0 \pm 0.7$ & - & - & - & - \\
$E_{\mathrm{cy}}$ & - & - & - & $0.907 \pm 0.009$ & - & - \\
$w_{\mathrm{cy}}$ & - & - & - & $0.54 \pm 0.02$ & - & - \\
$d_{\mathrm{cy}}$ & - & - & - & $1.32 \pm 0.02$ & - & - \\
$E_{\mathrm{G} 1}$ & - & - & $1.12 \pm 0.01$ & - & - & $1.00 \pm 0.01$ \\
$\sigma_{\mathrm{G} 1}$ & - & - & $0.39 \pm 0.01$ & - & - & $0.004 \pm 0.001$ \\
$\tau_{\mathrm{G} 1}$ & - & - & $1.41 \pm 0.03$ & - & - & $4_{-3}^{+51}$ \\
$E_{\mathrm{G} 2}$ & - & - & - & - & - & $1.29 \pm 0.03$ \\
$\sigma_{\mathrm{G} 2}$ & - & - & - & - & - & $0.18 \pm 0.03$ \\
$\tau_{\mathrm{G} 2}$ & - & - & - & - & - & $0.18 \pm 0.02$ \\
$k T_{1}$ & $0.140 \pm 0.005$ & $0.131 \pm 0.006$ & $0.1133 \pm 0.0005$ & $0.1312 \pm 0.0007$ & $0.07 \pm 0.01$ & $0.1382 \pm 0.0009$ \\
$k T_{2}$ & - & - & - & - & $0.15_{-0.01}^{+0.02}$ & - \\
Abs. Flux & $1.35_{-0.03}^{+0.02}$ & $1.36_{-0.05}^{+0.02}$ & $1.37 \pm 0.03$ & $1.37 \pm 0.03$ & $1.37 \pm 0.03$ & $1.37_{-0.04}^{+0.07}$ \\
Unab. Flux 1 & $13.3 \pm 0.3$ & $25.5 \pm 0.5$ & $224 \pm 5$ & $155 \pm 3$ & $520 \pm 20$ & $21.8 \pm 0.5$ \\
Unab. Flux 2 & - & - & - & - & $14.5 \pm 0.7$ & - \\
$R_{1}$ & $6 \pm 4$ & $10 \pm 6$ & $40 \pm 20$ & $30_{-20}^{+10}$ & $100 \pm 100$ & $8_{-4}^{+5}$ \\
$R_{2}$ & - & - & - & - & $6_{-3}^{+4}$ & - \\
$\chi_{\nu}^{2}$ (d.o.f.) & $1.41(153)$ & $1.28(152)$ & $1.21(150)$ & $1.19(150)$ & $1.19(151)$ & $1.09(147)$ \\
\hline \hline
\end{tabular}

Parameters fit to our data combined with the McLaughlin et al. (2007) data, fitting in the $0.5-2.0 \mathrm{keV}$ energy range. Fluxes are calculated in the $0.3-5.0 \mathrm{keV}$ energy range for direct comparison to the observation done by McLaughlin et al. (2007), reported in units of $10^{-13} \mathrm{erg} \mathrm{s}^{-1} \mathrm{~cm}^{-2} . n_{\mathrm{H}}$ is in units of $10^{22} \mathrm{~cm}^{-2}$ while $n_{\mathrm{O}}$ and $n_{\mathrm{Ne}}$ are in solar units (assuming solar abundance from Lodders (2003)). The photoelectric cross-section of Verner et al. (1996) has been used for all fits. The values of $k T$ (blackbody temperature), $E_{\mathrm{G}}$ (Gaussian line energy), $\sigma_{\mathrm{G}}$ (Gaussian line width), $E_{\mathrm{cy}}$ (cyclotron line energy) and $w_{\mathrm{cy}}$ (cyclotron line width) are in units of $\mathrm{keV} . R_{1}$ (blackbody emission radius at infinity assuming a $3.6 \mathrm{kpc}$ distance) and $R_{2}$ (blackbody hotspot emission radius at infinity in the two blackbody model, also assuming a $3.6 \mathrm{kpc}$ distance) are in units of $\mathrm{km}$. The Gaussian line depth $\tau_{\mathrm{G}}$ and fundamental cyclotron line depth $d_{\mathrm{cy}}$ are dimensionless. Errors are at the $1 \sigma$ confidence level. XSPEC models used are (from left to right): vphabs*bbody, vphabs*bbody, vphabs*gabs*bbody, vphabs*cyclabs*bbody, vphabs*(bbody+bbody), and vphabs*gabs*gabs*bbody. 
Table 3.3: Radio Parameters

\begin{tabular}{lcccc}
\hline \hline & Parkes 1 & Effelsberg & GBT & Parkes 2 \\
\hline MJD span & $54556.67-54557.00$ & $54557.10-54557.33$ & $54557.33-54557.65$ & $54557.74-54557.78$ \\
Center Freq. (GHz) & 1.4 & 1.4 & 1.9 & 1.4 \\
Bandwidth (MHz) & 256 & 80 & 600 & 256 \\
No. of frequency channels & 512 & 1 & 768 & 512 \\
Sampling Time $(\mu \mathrm{s})$ & 100 & 46000 & 81.92 & 100 \\
Observation Length $(\mathrm{hr})$ & 7.9 & 5.5 & 7.7 & 1.0 \\
$\beta_{\text {dig }}$ & 1.25 & 1.00 & 1.16 & 0.67 \\
$G$ (K/Jy) & 0.67 & 1.5 & 1.9 & 39 \\
$T_{\text {sys }}(\mathrm{K})$ & 39 & 27 & 29 & 320 \\
$\sigma_{S}(\mathrm{mJy})$ & 320 & 6.6 & 56 & 100 \\
$\sigma_{1 \mathrm{~ms}}(\mathrm{mJy})$ & 100 & 45 & 16.2 & \\
\hline
\end{tabular}

Radio observation parameters. See Section 3.3 for details.

\subsection{Radio Observations and Analysis}

Radio observations were carried out contemporaneously with the XMM-Newton satellite observations. The first radio observations were performed with the $64-\mathrm{m}$ Parkes radio telescope located in NSW, Australia using the multibeam receiver. After PSR J1819-1458 set at Parkes, we continued observing the source with the 100-m Effelsberg radio telescope located in Effelsberg, Germany. Just before the Effelsberg observations ended, we started observing PSR J1819-1458 with the 105m GBT in Green Bank, WV. The GBT measurements were followed up once again with the Parkes radio telescope for the remaining hour of the scheduled $X M M$ Newton observations. The durations and parameters of each radio observation are summarized in Table 3.3.

Radio pulses were first searched for by dedispersing the GBT and Parkes telescope data both at the DM of PSR J1819-1458, $196.0 \mathrm{~cm}^{-3} \mathrm{pc}$, and with zero dispersion using the SIGPROCpulsar processing package. Zero-DM time series were created for the GBT and Parkes telescope data to help discriminate pulses from ter- 
restrial radio sources. We could not dedisperse the Effelsberg telescope data because it had only one frequency channel. The radiometer noise, which is the rms deviation of the time series in flux density units, determines the sensitivity of each observation and is given by Equation 2.3. In this case, the correction factor accounting for the loss in sensitivity due to digitization is $1.25,1.16$, and 1.00 for the 1-, 2-, and 16-bit digitization of the Parkes, GBT, and Effelsberg telescopes, respectively. As we did in Chapter 2, we included the scaled 408-MHz sky temperatures of Haslam et al. (1981) assuming a spectral index of -2.6 (Lawson et al., 1987) in the $T_{\text {sys }}$ values quoted in Table 3.3. The parameters for each observation are detailed in Table 3.3. The effective sampling time of the Effelsberg telescope of $46 \mathrm{~ms}$ listed in Table 3.3 is the dispersion delay of the pulse over the single frequency channel's bandwidth of its receiver. Since this effective sampling time makes the Effelsberg telescope's radiometer noise misleadingly lower, we also provide a modified radiometer noise for comparison, $\sigma_{1 \mathrm{~ms}}$, which uses $t_{\mathrm{samp}}=1 \mathrm{~ms}$. Radio pulses that were detected with higher $\mathrm{S} / \mathrm{N}$ at the DM of the source than at zero DM (for the GBT and Parkes telescope data), exceeded the radiometer noise by a factor of five considering the false-alarm statistics, and were in phase with the radio ephemeris were considered real.

The times of arrival of the pulses from the RRAT were converted to barycentered arrival times at infinite frequency using TEMPO and the X-ray derived position. The folded solar system barycentered times are shown in the middle panel of Figure 3.1, which was created by finding the phase of each pulse using the radio ephemeris and then binning all the radio pulse arrival times into a 2048 bin his- 
togram. While individual radio pulses of PSR J1819-1458 typically consists of a single narrow pulse, the averaged radio pulse shape, shown in the bottom panel of Figure 3.1, has three separate components (Lyne et al., 2009; Karastergiou et al., 2009), a center component of more fainter pulses and two outer components made of fewer brighter pulses. Each outer component is $\sim 45 \mathrm{~ms}$ apart from the center component, much smaller than 1 of the 10 bins in the top panel of Figure 3.1, and does not affect the correlation analysis in Section 3.4 since only correlations greater than one spin period are considered. We detected 165 radio pulses in the first Parkes observation (i.e. 21 pulses $\mathrm{hr}^{-1}$ ), 64 pulses in the Effelsberg observation (i.e. 12 pulses $\mathrm{hr}^{-1}$ ), 673 pulses in the GBT observation (i.e. 90 pulses $\mathrm{hr}^{-1}$ ), and 29 pulses in the second Parkes observation (i.e. 29 pulses $\mathrm{hr}^{-1}$ ) for 931 radio pulses (bottom panel of Figure 3.3).

\subsection{Correlation of Radio Pulses and X-ray Photons}

For the correlation analysis we only considered X-ray photons from the 0.5-2.6 keV energy range determined in Section 3.2.1, shown as the dashed line in Figure 3.3. Analysis of the X-ray events within the GTIs satisfying either PATTERN $=0$ (i.e. allowing for only single events) as well as PATTERN $\leq 12$ was performed to see if this had any effect on the result. The PATTERN $=0$ requirement, not shown, yielded 4166 PN events, 1425 MOS1 events, and 1512 MOS2 events for a total of 7103 detections. and PATTERN $\leq 12$ requirement, shown in Figure 3.4, yielded 5692 PN events, 1705 MOS1 events, and 1767 MOS2 events for 


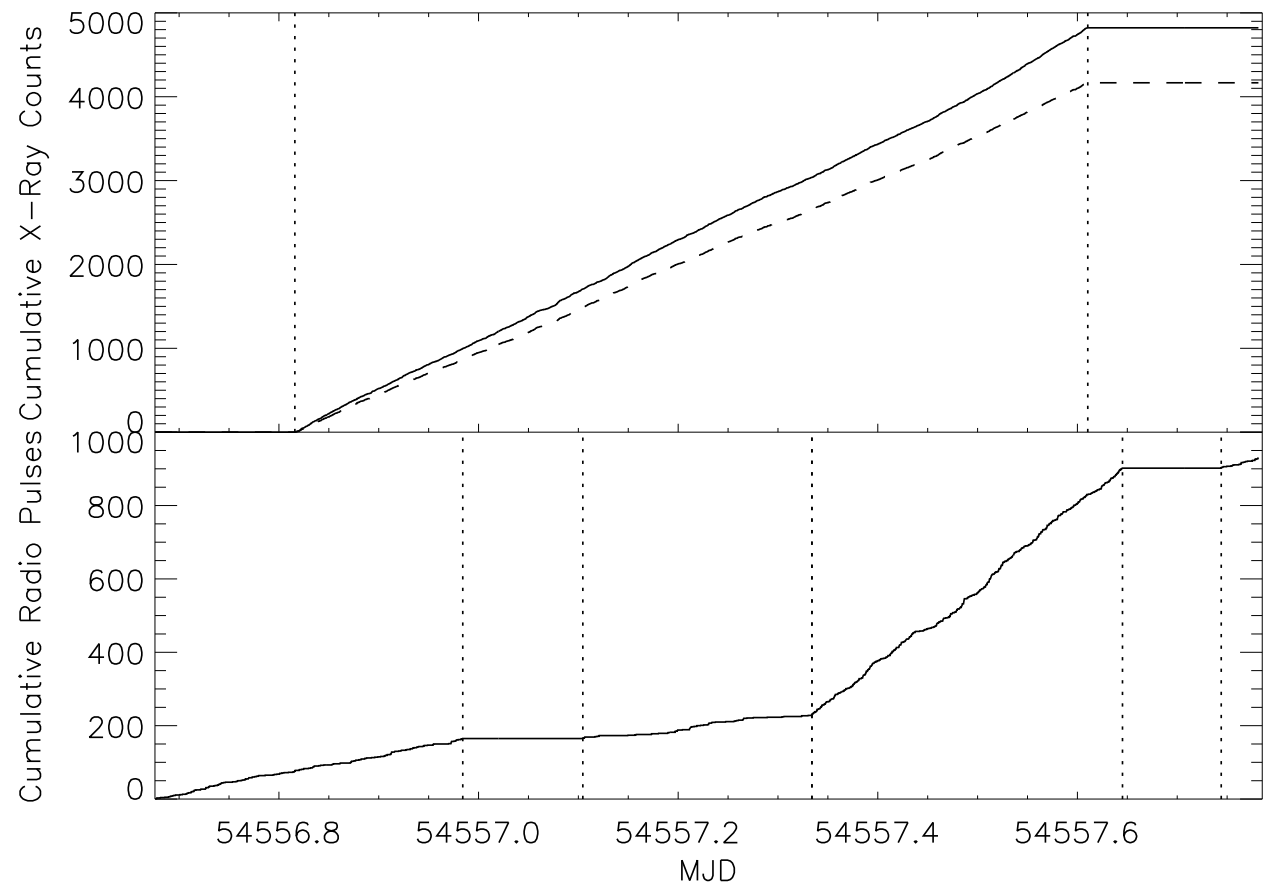

Figure 3.3: Top: Cumulative count of X-ray photon detections from PSR J1819-1458. The solid line represents all X-ray photons while the dashed only includes the photons with energies within the range $0.5-2.6 \mathrm{keV}$. Dashed vertical lines designate the beginning and end of the GTIs. In both cases, we only include photons from the source region described in Section 3.2.1. Bottom: $\mathrm{Cu}-$ mulative radio pulses detected by the following radio telescopes over time - Parkes, Effelsberg, GBT, and then Parkes again. Dashed vertical lines indicate the beginnings and endings of the radio telescopes' observing time. Different radio observing frequencies and sensitivities bring about the different slopes of the cumulative radio pulse distribution. The two flat regions of the distribution are attributed to the times when the pulsar was not observed. 
a total of 9164 detections.

The radio coverage described in Section 3.3 was not continuous due to two gaps - one between the first Parkes and Effelsberg observation and one between the GBT and second Parkes observation. The second Parkes telescope observation was contemporaneous with our $X M M$-Newton observation, but that portion of the $X M M-N e w t o n$ data was completely excluded by the GTIs. Due to the discontinuous radio and X-ray observation coverage as well as differences in radio telescope sensitivities, the distribution of time delays between X-ray detections and radio pulse detections is non-Gaussian. In order to measure the significance of any correlations between detected radio pulses and X-ray photons from PSR J1819-1458, we created a series of random X-ray photon distributions that would be consistent with the discontinuous coverage. We distributed the photon times throughout the GTIs, sampling from a flat (random) distribution. We created an array of $10^{4}$ random X-ray distributions to then compare to the radio pulse arrival times, in addition to the comparison with the $X M M-N e w t o n$ data.

We calculated the number of X-ray events detected by the PN and MOS cameras coincident with detected radio pulses at different lag times (see Figure 3.4). The lag time for each X-ray photon was calculated as the time elapsed between the X-ray detection time and its nearest detected radio pulse, either before or after the $\mathrm{X}$-ray detection. In this case, an X-ray detection was considered coincident if there was a radio pulse detected within some specified window of time, e.g. for a window of ten periods an X-ray photon was counted as coincident if there was at least one radio pulse detected within $10 \times 4.26 \mathrm{~s}=42.6 \mathrm{~s}$ of either before or after the $\mathrm{X}$-ray 


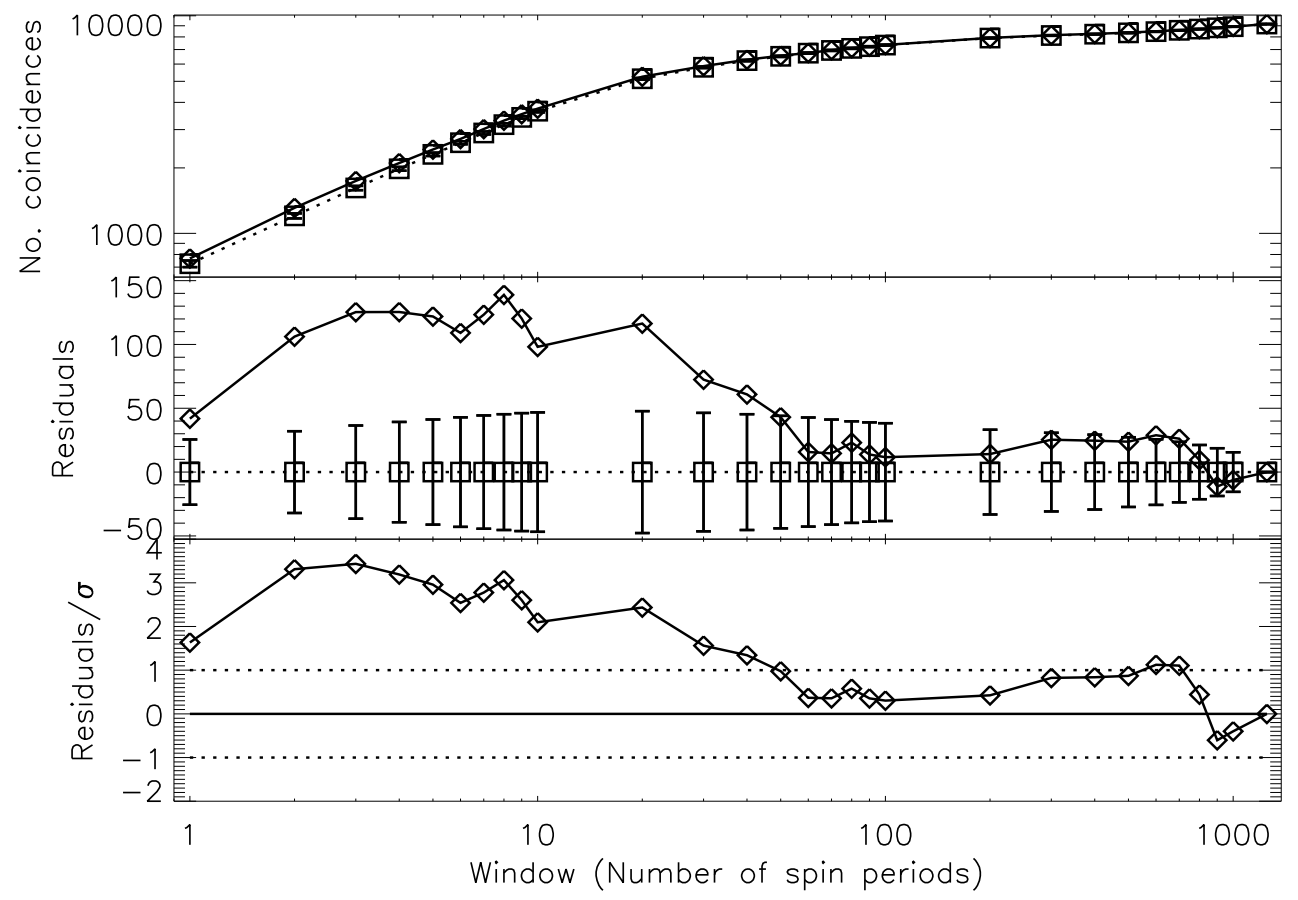

Figure 3.4: Top: Comparison of the number of PATTERN $\leq 12 \mathrm{X}$-ray photons from PSR J1819-1458 within the 0.5-2.6 keV energy range of the PN and MOS cameras that are coincident with a radio pulse within a given search window. X-ray photons coincident with the radio pulses are represented by diamonds and a solid line while the results of our simulation are represented by the squares (mean coincident photons of the simulations), vertical bars (standard deviation of coincident photons of the simulations) and the dotted line. Middle: The difference in the number of coincident photons for each window size in the data and the mean of the simulations. Bottom: As the middle plot, normalized by the standard deviation of the simulations. Here the horizontal dotted line indicates one standard deviation of the simulated random sets. 
event. This was also done for the array of simulated random sets. Then the mean and standard deviation of these sets for each lag window were calculated (the squares with vertical error bars in Figure 3.4). Differences between the data and simulated random sets are shown on the middle plot of Figure 3.4. Finally, the differences were divided by the standard deviations of the simulated random sets, shown in the bottom plot of Figure 3.4. For most lag window sizes, the number of coincident X-ray photons in the data exceeds the number of coincident X-ray photons in the simulated random sets. The largest deviation between the data and the simulations is $3.2 \sigma$ and $3.4 \sigma$ at $3 P \approx 12.8 \mathrm{~s}$ for the PATTERN $=0$ and PATTERN $\leq 12$ cases, respectively. Specifically, there were 1352 coincident X-ray detections but a mean of only 1262 coincident photons from the simulated random sets with a standard deviation of 32 photons with a $3 P$ window size for the PATTERN $=0$ case and 1742 coincident X-ray detections but only a mean of 1617 coincident photons from the simulated random sets with a standard deviation of 36 photons with a $3 P$ window size for the PATTERN $\leq 12$ case. Of our $10^{4}$ random sets, only 46 sets had a deviation exceeding $3.4 \sigma$ for one or more window sizes, the probability of this occurring by chance is then $0.46 \%$. Note that as the window size gets large enough, the data and simulated data sets converge once all the photons are considered coincident.

To help us gauge the significance of these deviations of the data versus randomized times, we also did the same analysis for another source on the same CCD in the field of view of XMM-Newton, 2XMMi J181928.8-145202, ${ }^{7}$ (see Figure 3.5). This source was not visible on the MOS1 detector, but we only considered the PN

\footnotetext{
${ }^{7}$ http://xmmssc-www.star.le.ac.uk/Catalogue/2XMMi/
} 
detector for this comparison. In this case, the randomized times are coincident more often than the real data, with the largest deviation peaking at $2.7 \sigma$ below the mean of the simulations at $10 P=42.63 \mathrm{~s}$. Of these $10^{4}$ random sets, 408 sets had a deviation exceeding $2.7 \sigma$ for one or more window sizes, the probability of this occurring by chance is then $4.08 \%$.

The KS test was used to determine the degree to which the X-ray data set itself differs from a random distribution. In this case we used both the numerical recipes ksone, which compares a single data set to an analytical distribution, and kstwo, which compares two data sets to one another. When comparing the combined PN and MOS detections to a flat distribution throughout the GTIs, the KS statistic from ksone is 0.14 (note that small values indicate the set is significantly different from the distribution). When we compared our $10^{4}$ simulated random sets to the distribution with ksone, we found a mean KS statistic of $0.5 \pm 0.3$, where the \pm 0.3 represents the standard deviation of the $10^{4}$ sets. We also compared the PN and MOS detections to the array of simulated random sets using kstwo. In this case, the mean KS statistic of these comparisons is $0.3 \pm 0.3$. We compared the radio pulse detections at each observation to a random distribution. The KS statistic from ksone was 0.77, 0.07, 0.09, and 0.23 for the first Parkes, Effelsberg, GBT, and second Parkes observations, respectively. We then compared each observation's pulse detections to $10^{4}$ simulated random sets with flat distributions containing the same number of pulse detections using kstwo. The mean KS statistic of these comparisons is $0.6 \pm 0.3,0.5 \pm 0.3$, $0.5 \pm 0.3$, and $0.5 \pm 0.3$ for the first Parkes, Effelsberg, GBT, and second Parkes observations, respectively. These statistics show that individually the X-ray photons 


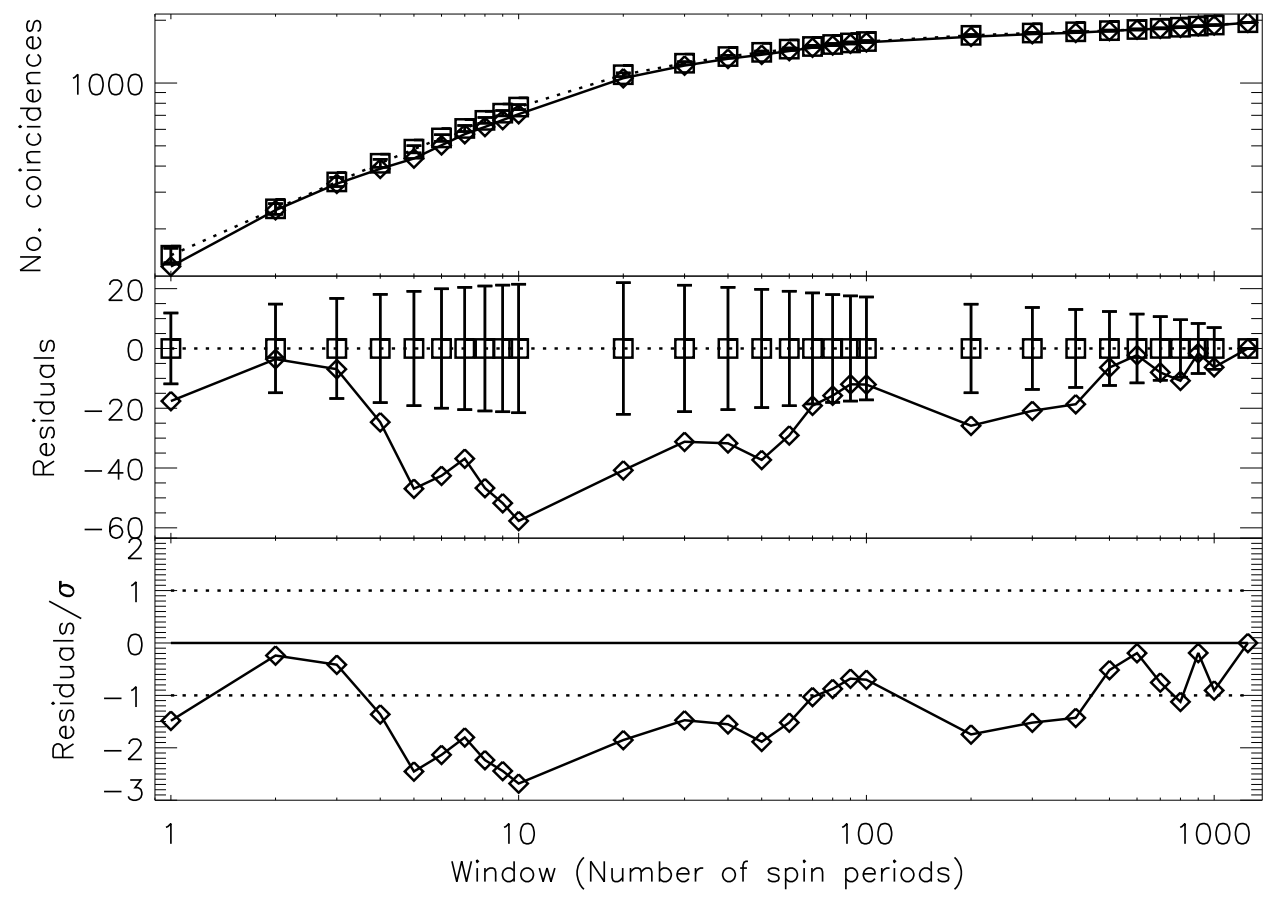

Figure 3.5: Top: Comparison of the number of X-ray photons from 2XMMi J181928.8-145202 within the 0.5-2.6 keV energy range from the PN detector only that are coincident with a radio pulse within a given search window. $\mathrm{X}$-ray photons coincident with the radio pulses are represented by diamonds and a solid line while the results of $10^{4}$ simulations are represented by the boxes (mean coincident photons of the simulations), vertical bars (standard deviation of coincident photons of the simulations) and the dotted line. Middle: The difference between the number of coincident photons for each window size in the data and the mean of the simulations. Bottom: As in the middle plot, normalized by the standard deviation of the simulations. Here the horizontal dotted line indicates one standard deviation of the simulated random sets. 
and the radio pulse detections are consistent with random distributions.

\subsection{Conclusions}

We have observed concurrent X-ray and radio pulsations from PSR J1819-1458. The peak of the X-ray profile is offset from the radio profile by $0.02 \pm 0.01$ in phase, which means they occur at the same phase within the timing resolution of $X M M$ Newton (73.4 ms, or 0.017 of the period). There is also evidence of a second sine-wave at twice the rotational frequency of the radio pulses and aligned with the X-ray profile peak, suggesting X-ray emission from the other pole of the neutron star and befitting a two blackbody model with both poles as hotspots. This is consistent with radio polarization observations that show that PSR J1819-1458 could be an orthogonal rotator (the angle between the pulsar's rotational axis and its magnetic dipole axis, $\alpha$, is not well-constrained, but most likely has a value near $90^{\circ}$; see Karastergiou et al., 2009).

The spectrum is consistent with a thermal emitter with a broad absorption line, possibly composed of two different lines around $\sim 1.0$ and $\sim 1.3 \mathrm{keV}$. Coupled with the detection of the absorption seen in a previous XMM-Newton observation (McLaughlin et al., 2007) and in the Chandra data (Rea et al., 2005; Camero-Arranz et al., 2013), we are certain of its astrophysical nature. If the line is due to proton resonant cyclotron scattering, then the cyclotron absorption line at $0.907 \mathrm{keV}$ (BB $\times$ Cyclotron in Table 3.2) implies a dipole magnetic field strength of $1.9 \times 10^{10} \mathrm{~T}$. If the absorption line is due to electron resonant cyclotron scattering, then the dipole 
magnetic field strength would be closer to $B_{\mathrm{S}}=1 \times 10^{7} \mathrm{~T}$. The surface dipole magnetic field strength estimate is proportional to the cosecant of $\alpha$ (see Equation 1.8). The surface dipole magnetic field strength is then consistent with the cyclotron proton resonant scattering model for $\alpha=15^{\circ}$. As we have described above there is evidence that $\alpha$ may be closer to $90^{\circ}$, though $\alpha$ is not well constrained. The inferred $\alpha$ for the electron cyclotron case is undefined since the implied dipole magnetic field strength of the model would be weaker than the surface dipole magnetic field strength estimate. This makes the electron cyclotron model unlikely.

We fit a blackbody temperature of $k T \sim 0.14 \mathrm{keV}$, slightly higher than what is expected from fast cooling models for high magnetic field pulsars (Aguilera et al., 2008; Pons et al., 2009). This relation, however, assumes the spin-down age $\left(\tau_{\mathrm{c}}\right.$, see Equation 1.7) to be correct, which might not be true (Noutsos et al., 2013), especially given the unusual glitch behavior of PSR J1819-1458 (Lyne et al., 2009). It is also interesting to consider that the derived X-ray luminosity from our best-fit model, a blackbody model with two Gaussian absorption lines, is $L_{0.3-5.0 \mathrm{keV}} \sim 3 \times 10^{26} \mathrm{~W}$, which exceeds the pulsar's spin-down luminosity by a factor of $\sim 10$. The $>25 \%$ uncertainty in the distance estimate, however, lends an even larger uncertainty to the derived X-ray luminosity estimate. The temperature and possibly high luminosity, combined with the unusual glitch activity, suggests that it could be a transitional object between pulsars and magnetars.

Our KS test results show that both the X-ray photon and radio pulse detections are consistent with random distributions. However, we have shown that the X-ray photon and radio pulse detections may be correlated on timescales of less than 10 
pulsar spin periods, where we measured a $3.4 \sigma$ deviation in our data from random distributions. As mentioned in Section 3.1, this tentative correlation suggests a link between the physical process producing the radio pulses and the heating of the polar-cap and represents the first enhancement of X-ray emission associated with radio pulse variability.

As introduced in Section 1.4.3 and discussed in Section 1.5.5, Zhang et al. (2007) proposed two interpretations which may explain the relationship between nulling pulsars, RRATs, and conventional radio pulsars. Their first model interpreted RRATs and nulling pulsars as dead pulsars that sporadically re-activate when coherent emission and pair production conditions are met. Their second model interpreted RRATs' behavior as a complement to nulling pulsars undergoing a reversal of radio emission direction. Zhang et al. proposed that X-ray observations may help discern between the two interpretations and specifically mention PSR J1819-1458 as fitting within the re-activated dead pulsar model because of its apparent lack of a non-thermal component in its X-ray spectrum (Reynolds et al., 2006; Gaensler et al., 2007). Even though we are currently unable to constrain a power-law tail, the tentative correlation between the radio pulse and $\mathrm{X}$-ray photon detection times suggests the reactivation model for PSR J1819-1458. 


\section{Chapter 4}

\section{Conclusions}

This work has introduced pulsars, described pulsar emission mechanism fundamentals, the different categories of pulsars including RRATs, presented new RRAT properties including radio pulse amplitude distributions as well as radio spectra, comparing and contrasting them to other pulsar populations, and presented simultaneous observations and analysis of radio and X-ray detections of PSR J1819-1458.

In Chapter 1 we laid the foundation for pulsar fundamentals. We described the basic characteristics of pulsars: our current understanding and how we derive pulsar parameters such as distance and magnetic field strength. This chapter explored the physics of radio and X-ray emission mechanisms and describing different regions of the pulsar magnetosphere where the emission may arise, e.g. the polar gap and outer gap, in order to set up the multiwavelength studies done for PSR J1819-1458 in Chapter 3. The introduction also described the different categories of pulsars: normal pulsars, millisecond pulsars (MSPs), giant pulsing pulsars, magnetars, nulling pulsars and RRATs. The unique properties of each of the populations motivates the analysis done in Chapter 2, where RRAT statistics are compared and contrasted to the statistics of other categories of pulsars, as well as the comparisons of the multiwavelength studies of PSR J1819-1458 to other multiwavelength studies of giant-pulsing pulsars and magnetars in Chapter 3 . 
The discovery of RRATs has been motivating single pulse searches of both new and archived data in additional to the traditional periodicity-based searches using Fourier techniques. Since the initial discovery of eleven RRATs in the PMPS, many more RRATs have been discovered even in the follow-up re-analysis of the PMPS itself. As single pulse detection algorithms become more robust, which are beginning to include artificial intelligence search algorithms, an explosion of the RRAT population is expected. If all RRATs are truly nulling (i.e., some pulses have a flux density of zero), Keane \& Kramer (2008) predict the RRATs Galactic population may be equal to if not greater than that of normal pulsars which brings about discrepancies in the neutron star birthrate and core-collapse supernova rates.

It is still not understood how RRATs relate to other types of pulsars and whether they do indeed provide a "missing link" within the evolution of neutron stars. For the same reason RRATs are difficult to detect, it is also difficult to determine their position as well as their $P$ and $\dot{P}$ values, which in turn would provide the derived parameters described in Chapter 1. It is currently unknown if other RRATs have glitches similar to PSR J1819-1458 or if it is an anomaly; PSR J1819-1458 has the selection effect of being the most reliable RRAT to detect with the highest burst rate; whether other RRATs have glitches and/or anti-glitches will give insight to their possible connection to magnetars.

RRATs may break down into multiple subcategories, e.g. those that are only actively emitting pulses part of the time, those that are emitting some pulses too weak to detect with current instruments, and those that are reversing their radio emission direction part of the time. As more data become available, more robust 
statistics can be made. Due to their sparse emission, however, a much larger amount of telescope time is required to study RRATs than compared to other pulsars.

New interferometric arrays of radio telescopes being built such as the LowFrequency Array have omni-directional antennas which will allow more time to observe the dynamic sky that will in turn allow more transient detections. The Square Kilometer Array in development in Australia and South Africa will have a $\sim 1 \mathrm{~km}$ effective collecting area and will surpass all current radio telescope sensitivities. Future technology and studies will allow not only the detection of weaker RRATs, but will also allow us to probe current RRATs with better sensitivities that will extend our understanding of these objects and will ultimately allow us to see if some RRATs are truly transient. 


\section{Bibliography}

Aguilera D. N., Pons J. A., Miralles J. A., 2008, A\&A, 486, 255

Archibald R. F., Kaspi V. M., Ng C.-Y., Gourgouliatos K. N., Tsang D., Scholz P., Beardmore A. P., Gehrels N., Kennea J. A., 2013, Nature, 497, 591

Argyle E., Gower J. F. R., 1972, ApJ, 175, L89

Baade W., Zwicky F., 1934, PNAS, 20, 254

Backer D. C., 1970, Nature, 228, 42

Backer D. C., Kulkarni S. R., Heiles C., Davis M. M., Goss W. M., 1982, Nature, 300,615

Bai X.-N., Spitkovsky A., 2010, ApJ, 715, 1282

Bałucińska-Church M., McCammon D., 1992, ApJ, 400, 699

Bhat N. D. R., Cordes J. M., Camilo F., Nice D. J., Lorimer D. R., 2004, ApJ, 605, 759

Bhattacharya D., van den Heuvel E. P. J., 1991, Phys. Rep., 203, 1

Biggs J. D., 1992, ApJ, 394, 574

Bilous A. V., Kondratiev V. I., McLaughlin M. A., Ransom S. M., Lyutikov M., Mickaliger M., Langston G. I., 2011, ApJ, 728, 110

Bisnovatyi-Kogan G. S., Komberg B. V., 1974, Sov. Astron., 18, 217

Blandford R. D., 1975, MNRAS, 170, 551

Brinklow A., 1989, PhD thesis, The University of Manchester

Burgay M., McLaughlin M. A., Reynolds S. P., 2007, in di Salvo T., Israel G. L., Piersant L., Burderi L., Matt G., Tornambe A., Menna M. T., eds, The Multicolored Landscape of Compact Objects and Their Explosive Origins Vol. 924 of American Institute of Physics Conference Series, On the debated nature of Rotating RAdio Transients. pp 607-612

Burke-Spolaor S., 2013, in IAU Symposium Vol. 291 of IAU Symposium, Rotating Radio Transients and their place among pulsars. pp 95-100

Burke-Spolaor S., Bailes M., 2010, MNRAS, 402, 855

Cairns I. H., Johnston S., Das P., 2001, ApJ, 563, L65

Cairns I. H., Johnston S., Das P., 2004, MNRAS, 353, 270 
Camero-Arranz A., Rea N., Bucciantini N., McLaughlin M. A., Slane P., Gaensler B. M., Torres D. F., Stella L., de Oña E., Israel G. L., Camilo F., Possenti A., 2013, MNRAS, 429, 2493

Camilo F., Ransom S. M., Halpern J. P., Reynolds J., 2007, ApJ, 666, L93

Camilo F., Ransom S. M., Halpern J. P., Reynolds J., Helfand D. J., Zimmerman N., Sarkissian J., 2006, Nature, 442, 892

Camilo F., Reynolds J., Johnston S., Halpern J. P., Ransom S. M., 2008, ApJ, 679, 681

Cheng A. F., Ruderman M. A., 1977, ApJ, 216, 865

Cheng K. S., Ho C., Ruderman M., 1986, ApJ, 300, 500

Chiang J., Romani R. W., 1992, ApJ, 400, 629

Chiang J., Romani R. W., 1994, ApJ, 436, 754

Cognard I., Shrauner J. A., Taylor J. H., Thorsett S. E., 1996, ApJ, 457, L81

Collins S., Shearer A., Stappers B., Barbieri C., Naletto G., Zampieri L., Verroi E., Gradari S., 2012, in Griffin E., Hanisch R., Seaman R., eds, IAU Symposium Vol. 285 of IAU Symposium, Crab Pulsar: Enhanced Optical Emission During Giant Radio Pulses. pp 296-298

Cordes J. M., Lazio T. J. W., 2002, ArXiv Astrophysics e-prints

Cordes J. M., McLaughlin M. A., 2003, ApJ, 596, 1142

Cordes J. M., Shannon R. M., 2008, ApJ, 682, 1152

Cui B., Boyles J., McLaughlin M., Palliyaguru N., 2013, in IAU Symposium Vol. 291 of IAU Symposium, New timing solutions for RRATs. pp 366-368

Daugherty J. K., Harding A. K., 1982, ApJ, 252, 337

Daugherty J. K., Harding A. K., 1986, ApJ, 309, 362

Deneva J. S., Cordes J. M., McLaughlin M. A., Nice D. J., Lorimer D. R., et al., 2009, ApJ, 703, 2259

Deutsch A. J., 1955, Annales d'Astrophysique, 18, 1

Dickey J. M., Lockman F. J., 1990, ARA\&A, 28, 215

Duncan R. C., Thompson C., 1992, ApJ, 392, L9

Dyks J., Rudak B., 2003, ApJ, 598, 1201

Edwards R. T., Bailes M., van Straten W., Britton M. C., 2001, MNRAS, 326, 358 
Esamdin A., Zhao C. S., Yan Y., Wang N., Nizamidin H., Liu Z. Y., 2008, MNRAS, 389, 1399

Espinoza C. M., Lyne A. G., Kramer M., Manchester R. N., Kaspi V. M., 2011, apjl, 741, L13

Gaensler B. M., McLaughlin M., Reynolds S., Borkowski K., Rea N., Possenti A., Israel G., Burgay M., Camilo F., Chatterjee S., Kramer M., Lyne A., Stairs I., 2007, Ap\&SS, 308, 95

Garmire G. P., Bautz M. W., Ford P. G., Nousek J. A., Ricker Jr. G. R., 2003, in Truemper J. E., Tananbaum H. D., eds, Society of Photo-Optical Instrumentation Engineers (SPIE) Conference Series Vol. 4851 of Society of Photo-Optical Instrumentation Engineers (SPIE) Conference Series, Advanced CCD imaging spectrometer (ACIS) instrument on the Chandra X-ray Observatory. pp 28-44

Goldreich P., Julian W. H., 1969, ApJ, 157, 869

Hankins T. H., Kern J. S., Weatherall J. C., Eilek J. A., 2003, Nature, 422, 141

Harding A. K., DeCesar M. E., Miller M. C., Kalapotharakos C., Contopoulos I., 2011, ArXiv e-prints

Harding A. K., Muslimov A. G., Zhang B., 2002, ApJ, 576, 366

Haslam C. G. T., Klein U., Salter C. J., Stoffel H., Wilson W. E., Cleary M. N., Cooke D. J., Thomasson P., 1981, A\&A, 100, 209

He C., Ng C.-Y., Kaspi V. M., 2013, ApJ, 768, 64

Hesse K. H., Wielebinski R., 1974, A\&A, 31, 409

Hessels J. W. T., Ransom S. M., Kaspi V. M., Roberts M. S. E., Champion D. J., Stappers B. W., 2008, in Bassa C., Wang Z., Cumming A., Kaspi V. M., eds, 40 Years of Pulsars: Millisecond Pulsars, Magnetars and More Vol. 983 of American Institute of Physics Conference Series, The GBT350 Survey of the Northern Galactic Plane for Radio Pulsars and Transients. pp 613-615

Hessels J. W. T., Ransom S. M., Stairs I. H., Freire P. C. C., Kaspi V. M., Camilo F., 2006, Science, 311, 1901

Hewish A., Bell S. J., Pilkington J. D. H., Scott P. F., Collins R. A., 1968, Nature, 217,709

Hohle M. M., Haberl F., Vink J., de Vries C. P., Neuhäuser R., 2012, MNRAS, 419, 1525

Jenet F. A., Anderson S. B., 1998, PASP, 110, 1467

Johnston S., 2004, MNRAS, 348, 1229 
Johnston S., Romani R., 2002, MNRAS, 332, 109

Kalapotharakos C., Harding A. K., Kazanas D., Contopoulos I., 2012, ApJ, 754, L1

Kalapotharakos C., Kazanas D., Harding A., Contopoulos I., 2012, ApJ, 749, 2

Kalberla P. M. W., Burton W. B., Hartmann D., Arnal E. M., Bajaja E., Morras R., Pöppel W. G. L., 2005, A\&A, 440, 775

Kaplan D. L., van Kerkwijk M. H., 2011, ApJ, 740, L30

Karastergiou A., Hotan A. W., van Straten W., McLaughlin M. A., Ord S. M., 2009, MNRAS, 396, L95

Kargaltsev O., Durant M., Misanovic Z., Pavlov G. G., 2012, Science, 337, 946

Keane E. F., Kramer M., 2008, MNRAS, 391, 2009

Keane E. F., Kramer M., Lyne A. G., Stappers B. W., McLaughlin M. A., 2011, MNRAS, 415, 3065

Keane E. F., Ludovici D. A., Eatough R. P., Kramer M., Lyne A. G., McLaughlin M. A., Stappers B. W., 2010, MNRAS, 401, 1057

Keane E. F., McLaughlin M. A., 2011, Bulletin of the Astronomical Society of India, 39,333

Kinkhabwala A., Thorsett S. E., 2000, ApJ, 535, 365

Knight H. S., 2006, Chinese Journal of Astronomy and Astrophysics Supplement, 6,020000

Knight H. S., Bailes M., Manchester R. N., Ord S. M., 2006, ApJ, 653, 580

Knight H. S., Bailes M., Manchester R. N., Ord S. M., Jacoby B. A., 2006, ApJ, 640,941

Kostyuk S. V., Kondratiev V. I., Kuzmin A. D., Popov M. V., Soglasnov V. A., 2003, Astronomy Letters, 29, 387

Kramer M., Johnston S., van Straten W., 2002, MNRAS, 334, 523

Kramer M., Karastergiou A., Gupta Y., Johnston S., Bhat N. D. R., Lyne A. G., 2003, A\&A, 407, 655

Kuiper L., Hermsen W., den Hartog P. R., Collmar W., 2006, ApJ, 645, 556

Lattimer J. M., Prakash M., 2001, ApJ, 550, 426

Lawson K. D., Mayer C. J., Osborne J. L., Parkinson M. L., 1987, MNRAS, 225, 307 
Lazaridis K., Jessner A., Kramer M., Stappers B. W., Lyne A. G., Jordan C. A., Serylak M., Zensus J. A., 2008, MNRAS, 390, 839

Levin L., Bailes M., Bates S., Bhat N. D. R., Burgay M., Burke-Spolaor S., D'Amico N., Johnston S., Keith M., Kramer M., Milia S., Possenti A., Rea N., Stappers B., van Straten W., 2010, ApJ, 721, L33

Li X.-D., 2006, ApJ, 646, L139

Lodders K., 2003, ApJ, 591, 1220

Lorimer D. R., Kramer M., 2005, Handbook of Pulsar Astronomy. Cambridge University Press

Lundgren S. C., Cordes J. M., Ulmer M., Matz S. M., Lomatch S., Foster R. S., Hankins T., 1995, ApJ, 453, 433

Luo Q., Melrose D., 2007, MNRAS, 378, 1481

Luo Q., Melrose D. B., 1992, MNRAS, 258, 616

Luo Q., Melrose D. M., 1995, ApJ, 452, 346

Lyne A. G., McLaughlin M. A., Keane E. F., Kramer M., Espinoza C. M., Stappers B. W., Palliyaguru N. T., Miller J., 2009, MNRAS, 400, 1439

Lyne A. G., Pritchard R. S., Graham-Smith F., Camilo F., 1996, Nature, 381, 497

Lyutikov M., 2007, MNRAS, 381, 1190

Lyutikov M., Blandford R. D., Machabeli G., 1999, MNRAS, 305, 338

Machabeli G. Z., Usov V. V., 1979, Sov. Astron. Lett., 5, 238

McLaughlin M. A., Lyne A. G., Keane E. F., Kramer M., Miller J. J., Lorimer D. R., Manchester R. N., Camilo F., Stairs I. H., 2009, MNRAS, 400, 1431

Magalhaes N. S., Miranda T. A., Frajuca C., 2012, apj, 755, 54

Manchester R. N., Lyne A. G., Camilo F., Bell J. F., Kaspi V. M., D’Amico N., McKay N. P. F., Crawford F., Stairs I. H., Possenti A., Morris D. J., Sheppard D. C., 2001, MNRAS, 328, 17

Maron O., Kijak J., Kramer M., Wielebinski R., 2000, A\&AS, 147, 195

McLaughlin M. A., Lyne A. G., Lorimer D. R., Kramer M., Faulkner A. J., Manchester R. N., Cordes J. M., Camilo F., Possenti A., Stairs I. H., Hobbs G., D'Amico N., Burgay M., O’Brien J. T., 2006, Nature, 439, 817

McLaughlin M. A., Rea N., Gaensler B. M., Chatterjee S., Camilo F., Kramer M., Lorimer D. R., Lyne A. G., Israel G. L., Possenti A., 2007, ApJ, 670, 1307 
Melrose D. B., 1992, Royal Society of London Philosophical Transactions Series A, 341,105

Melrose D. B., 1995, Journal of Astrophysics and Astronomy, 16, 137

Mickaliger M. B., Lorimer D. R., Boyles J., McLaughlin M. A., Collins A., Hough L., Tehrani N., Tenney C., Liska A., Swiggum J., 2012, ApJ, 759, 127

Mihara T., Makishima K., Ohashi T., Sakao T., Tashiro M., 1990, Nature, 346, 250

Muno M. P., Gaensler B. M., Clark J. S., de Grijs R., Pooley D., Stevens I. R., Portegies Zwart S. F., 2007, MNRAS, 378, L44

Muslimov A. G., Harding A. K., 2003, ApJ, 588, 430

Muslimov A. G., Harding A. K., 2004, ApJ, 606, 1143

Noutsos A., Schnitzeler D. H. F. M., Keane E. F., Kramer M., Johnston S., 2013, MNRAS, 430, 2281

Pons J. A., Miralles J. A., Geppert U., 2009, A\&A, 496, 207

Popov M. V., Soglasnov V. A., Kondrat'ev V. I., Bilous A. V., Sazankov S. V., Smirnov A. I., Kanevskii B. Z., Oreshko V. V., Ilyasov Y. P., 2008, Astronomy Reports, 52, 900

Popov M. V., Stappers B., 2007, A\&A, 470, 1003

Popov S. B., Prokhorov M. E., 2006, MNRAS, 367, 732

Press W. H., Flannery B. P., Teukolsky S. A., Vetterling W. T., 1986, Numerical Recipes: The Art of Scientific Computing. Cambridge University Press, Cambridge

Rea N., Esposito P., Pons J. A., Turolla R., Torres D. F., Israel G. L., Possenti A., Burgay M., et al., 2013, ApJ, 775, L34

Rea N., McLaughlin M. A., Gaensler B. M., Slane P. O., Stella L., Reynolds S. P., Burgay M., Israel G. L., Possenti A., Chatterjee S., 2009, ApJ, 703, L41

Rea N., Oosterbroek T., Zane S., Turolla R., Méndez M., Israel G. L., Stella L., Haberl F., 2005, MNRAS, 361, 710

Reynolds S. P., Borkowski K. J., Gaensler B. M., Rea N., McLaughlin M., Possenti A., Israel G., Burgay M., Camilo F., Chatterjee S., Kramer M., Lyne A., Stairs I., 2006, ApJ, 639, L71

Ritchings R. T., 1976, MNRAS, 176, 249

Romani R. W., 1996, ApJ, 470, 469 
Ruderman M. A., Sutherland P. G., 1975, ApJ, 196, 51

Sallmen S., Backer D. C., Hankins T. H., Moffett D., Lundgren S., 1999, ApJ, 517, 460

Serylak M., Stappers B. W., Weltevrede P., Kramer M., Jessner A., Lyne A. G., Jordan C. A., Lazaridis K., Zensus J. A., 2009, MNRAS, 394, 295

Shearer A., Stappers B., O'Connor P., Golden A., Strom R., Redfern M., Ryan O., 2003, Science, 301, 493

Shibazaki N., Murakami T., Shaham J., Nomoto K., 1989, Nature, 342, 656

Spitkovsky A., 2006, ApJ, 648, L51

Thorsett S. E., Chakrabarty D., 1999, ApJ, 512, 288

Turolla R., 2009, in Becker W., ed., Astrophysics and Space Science Library Vol. 357 of Astrophysics and Space Science Library, Isolated Neutron Stars: The Challenge of Simplicity. p. 141

Vasisht G., Gotthelf E. V., 1997, ApJ, 486, L129

Verner D. A., Ferland G. J., Korista K. T., Yakovlev D. G., 1996, ApJ, 465, 487

Wang N., Manchester R. N., Johnston S., 2007, MNRAS, 377, 1383

Watson M. G., Schröder A. C., Fyfe D., Page C. G., Lamer G., Mateos S., Pye J., et al., 2009, A\&A, 493, 339

Weisskopf M. C., Brinkman B., Canizares C., Garmire G., Murray S., Van Speybroeck L. P., 2002, PASP, 114, 1

Weltevrede P., Stappers B. W., Rankin J. M., Wright G. A. E., 2006, ApJ, 645, L149

Woods P. M., Kaspi V. M., Thompson C., Gavriil F. P., Marshall H. L., Chakrabarty D., Flanagan K., Heyl J., Hernquist L., 2004, ApJ, 605, 378

Woods P. M., Kouveliotou C., Finger M. H., Göğüş E., Wilson C. A., Patel S. K., Hurley K., Swank J. H., 2007, ApJ, 654, 470

Woods P. M., Kouveliotou C., Göğüş E., Finger M. H., Swank J., Smith D. A., Hurley K., Thompson C., 2001, ApJ, 552, 748

Yakovlev D. G., Pethick C. J., 2004, ARA\&A, 42, 169

Zhang B., Gil J., Dyks J., 2007, MNRAS, 374, 1103 